\title{
Framing and Context of the Report
}

\section{Coordinating Lead Authors:}

Nerilie Abram (Australia), Jean-Pierre Gattuso (France), Anjal Prakash (Nepal//ndia)

\section{Lead Authors:}

Lijing Cheng (China), Maria Paz Chidichimo (Argentina), Susan Crate (USA), Hiroyuki Enomoto (Japan), Matthias Garschagen (Germany), Nicolas Gruber (Switzerland), Sherilee Harper (Canada), Elisabeth Holland (Fiji), Raphael Martin Kudela (USA), Jake Rice (Canada), Konrad Steffen (Switzerland), Karina von Schuckmann (France)

\section{Contributing Authors:}

Nathaniel Bindoff (Australia), Sinead Collins (UK), Rebecca Colvin (Australia), Daniel Farinotti (Switzerland), Nathalie Hilmi (France/Monaco), Jochen Hinkel (Germany), Regine Hock (USA), Alexandre Magnan (France), Michael Meredith (UK), Avash Pandey (Nepal), Mandira Singh Shrestha (Nepal), Anna Sinisalo (Nepal/Finland), Catherine Sutherland (South Africa), Phillip Williamson (UK)

\section{Review Editors:}

Monika Rhein (Germany), David Schoeman (Australia)

\section{Chapter Scientists:}

Avash Pandey (Nepal), Bethany Ellis (Australia)

\section{This chapter should be cited as:}

Abram, N., J.-P. Gattuso, A. Prakash, L. Cheng, M.P. Chidichimo, S. Crate, H. Enomoto, M. Garschagen, N. Gruber, S. Harper, E. Holland, R.M. Kudela, J. Rice, K. Steffen, and K. von Schuckmann, 2019: Framing and Context of the Report. In: IPCC Special Report on the Ocean and Cryosphere in a Changing Climate [H.-O. Pörtner, D.C. Roberts, V. Masson-Delmotte, P. Zhai, M. Tignor, E. Poloczanska, K. Mintenbeck, A. Alegría, M. Nicolai, A. Okem, J. Petzold, B. Rama, N.M. Weyer (eds.)]. Cambridge University Press, Cambridge, UK and New York, NY, USA, pp. 73-129. https://doi.org/10.1017/9781009157964.003. 


\section{Table of contents}

Executive Summary

1.1 Why this Special Report?

77

Box 1.1: Major Components and Characteristics of the Ocean and Cryosphere 78

1.2 Role of the Ocean and Cryosphere in the Earth System

1.2.1 Ocean and Cryosphere in Earth's Energy, Water and Biogeochemical Cycles

1.2.2 Interactions Between the Ocean and Cryosphere

1.3 Time Scales, Thresholds and Detection of Ocean and Cryosphere Change

1.4 Changes in the Ocean and Cryosphere 83

1.4.1 Observed and Projected Changes in the Ocean . .83

1.4.2 Observed and Projected Changes in the Cryosphere

Cross-Chapter Box 1: Scenarios,

Pathways and Reference Periods 84

1.5 Risk and Impacts Related to Ocean and Cryosphere Change

Cross-Chapter Box 2: Key Concepts of Risk, Adaptation, Resilience and Transformation

1.5.1 Hazards and Opportunities for Natural Systems, Ecosystems, and Human Systems .......... 90

1.5.2 Exposure of Natural Systems, Ecosystems, and Human Systems 91

1.5.3 Vulnerabilities in Natural Systems, Ecosystems, and Human Systems

1.6 Addressing the Causes and Consequences of Climate Change for the Ocean and Cryosphere 92

1.6.1 Mitigation and Adaptation Options in the Ocean and Cryosphere

1.6.2 Adaptation in Natural Systems, Ecosystems, and Human Systems 94
1.7 Governance and Institutions 95

Cross-Chapter Box 3: Governance of the Ocean, Coasts and the Cryosphere under Climate Change 95

1.8 Knowledge Systems for Understanding and Responding to Change 99

1.8.1 Scientific Knowledge .........................................99

1.8.2 Indigenous Knowledge and Local Knowledge ... 102

1.8.3 The Role of Knowledge in People's Responses to Climate, Ocean and Cryosphere Change 102

Cross-Chapter Box 4: Indigenous

Knowledge and Local Knowledge

in Ocean and Cryosphere Change 103

1.9 Approaches Taken in this Special Report ………..... 106

1.9.1 Methodologies Relevant to this Report ……....... 106

1.9.2 Communication of Confidence in Assessment Findings 106

Cross-Chapter Box 5: Confidence and Deep Uncertainty 107

1.10 Integrated Storyline of this Special Report 110

References 116

Frequently Asked Questions

FAQ 1.1 How do changes in the ocean and cryosphere affect our life on planet Earth?

FAQ 1.2 How will changes in the ocean and cryosphere affect meeting the Sustainable Development Goals? 


\section{Executive Summary}

This special report assesses new knowledge since the IPCC 5th Assessment Report (AR5) and the Special Report on Global Warming of $1.5^{\circ} \mathrm{C}$ (SR15) on how the ocean and cryosphere have and are expected to change with ongoing global warming, the risks and opportunities these changes bring to ecosystems and people, and mitigation, adaptation and governance options for reducing future risks. Chapter 1 provides context on the importance of the ocean and cryosphere, and the framework for the assessments in subsequent chapters of the report.

All people on Earth depend directly or indirectly on the ocean and cryosphere. The fundamental roles of the ocean and cryosphere in the Earth system include the uptake and redistribution of anthropogenic carbon dioxide and heat by the ocean, as well as their crucial involvement of in the hydrological cycle. The cryosphere also amplifies climate changes through snow, ice and permafrost feedbacks. Services provided to people by the ocean and/or cryosphere include food and freshwater, renewable energy, health and wellbeing, cultural values, trade and transport. $\{1.1,1.2,1.5\}$

Sustainable development is at risk from emerging and intensifying ocean and cryosphere changes. Ocean and cryosphere changes interact with each of the United Nations Sustainable Development Goals (SDGs). Progress on climate action (SDG 13) would reduce risks to aspects of sustainable development that are fundamentally linked to the ocean and cryosphere and the services they provide (high confidence ${ }^{1}$ ). Progress on achieving the SDGs can contribute to reducing the exposure or vulnerabilities of people and communities to the risks of ocean and cryosphere change (medium confidence). $\{1.1\}$

Communities living in close connection with polar, mountain, and coastal environments are particularly exposed to the current and future hazards of ocean and cryosphere change. Coasts are home to approximately $28 \%$ of the global population, including around $11 \%$ living on land less than $10 \mathrm{~m}$ above sea level. Almost $10 \%$ of the global population lives in the Arctic or high mountain regions. People in these regions face the greatest exposure to ocean and cryosphere change, and poor and marginalised people here are particularly vulnerable to climate-related hazards and risks (very high confidence). The adaptive capacity of people, communities and nations is shaped by social, political, cultural, economic, technological, institutional, geographical and demographic factors. $\{1.1,1.5,1.6$, Cross-Chapter Box 2 in Chapter 1$\}$
Ocean and cryosphere changes are pervasive and observed from high mountains, to the polar regions, to coasts, and into the deep ocean. AR5 assessed that the ocean is warming (0 to $700 \mathrm{~m}$ : virtually certain ${ }^{2} ; 700$ to $2,000 \mathrm{~m}$ : likely), sea level is rising (high confidence), and ocean acidity is increasing (high confidence). Most glaciers are shrinking (high confidence), the Greenland and Antarctic ice sheets are losing mass (high confidence), sea ice extent in the Arctic is decreasing (very high confidence), Northern Hemisphere snow cover is decreasing (very high confidence), and permafrost temperatures are increasing (high confidence). Improvements since AR5 in observation systems, techniques, reconstructions and model developments, have advanced scientific characterisation and understanding of ocean and cryosphere change, including in previously identified areas of concern such as ice sheets and Atlantic Meridional Overturning Circulation (AMOC). $\{1.1,1.4,1.8 .1\}$

Evidence and understanding of the human causes of climate warming, and of associated ocean and cryosphere changes, has increased over the past 30 years of IPCC assessments (very high confidence). Human activities are estimated to have caused approximately $1.0^{\circ} \mathrm{C}$ of global warming above pre-industrial levels (SR15). Areas of concern in earlier IPCC reports, such as the expected acceleration of sea level rise, are now observed (high confidence). Evidence for expected slow-down of AMOC is emerging in sustained observations and from long-term palaeoclimate reconstructions (medium confidence), and may be related with anthropogenic forcing according to model simulations, although this remains to be properly attributed. Significant sea level rise contributions from Antarctic ice sheet mass loss (very high confidence), which earlier reports did not expect to manifest this century, are already being observed. $\{1.1,1.4\}$

Ocean and cryosphere changes and risks by the end-of-century (2081-2100) will be larger under high greenhouse gas emission scenarios, compared with low emission scenarios (very high confidence). Projections and assessments of future climate, ocean and cryosphere changes in the Special Report on the Ocean and Cryosphere in a Changing Climate (SROCC) are commonly based on coordinated climate model experiments from the Coupled Model Intercomparison Project Phase 5 (CMIP5) forced with Representative Concentration Pathways (RCPs) of future radiative forcing. Current emissions continue to grow at a rate consistent with a high emission future without effective climate change mitigation policies (referred to as RCP8.5). The SROCC assessment contrasts this high greenhouse gas emission future with a low greenhouse gas emission, high mitigation future (referred to as RCP2.6) that gives a two in three chance of limiting warming by the end of the century to less than $2^{\circ} \mathrm{C}$ above pre-industrial. \{Cross-Chapter Box 1 in Chapter 1\}

In this report, the following summary terms are used to describe the available evidence: limited, medium, or robust; and for the degree of agreement: low, medium or high. A level of confidence is expressed using five qualifiers: very low, low, medium, high and very high, and typeset in italics, for example, medium confidence. For a given evidence and agreement statement, different confidence levels can be assigned, but increasing levels of evidence and degrees of agreement are correlated with increasing confidence (see Section 1.9.2 and Figure 1.4 for more details).

2 In this report, the following terms have been used to indicate the assessed likelihood of an outcome or a result: Virtually certain 99-100\% probability, Very likely $90-100 \%$, Likely $66-100 \%$, About as likely as not 33-66\%, Unlikely 0-33\%, Very unlikely $0-10 \%$, and Exceptionally unlikely $0-1 \%$. Additional terms (Extremely likely: $95-100 \%$, More likely than not $>50-100 \%$, and Extremely unlikely $0-5 \%$ ) may also be used when appropriate. Assessed likelihood is typeset in italics, for example, very likely (see Section 1.9.2 and Figure 1.4 for more details). This Report also uses the term 'likely range' to indicate that the assessed likelihood of an outcome lies within the $17-83 \%$ probability range. 
Characteristics of ocean and cryosphere change include thresholds of abrupt change, long-term changes that cannot be avoided, and irreversibility (high confidence). Ocean warming, acidification and deoxygenation, ice sheet and glacier mass loss, and permafrost degradation are expected to be irreversible on time scales relevant to human societies and ecosystems. Long response times of decades to millennia mean that the ocean and cryosphere are committed to long-term change even after atmospheric greenhouse gas concentrations and radiative forcing stabilise (high confidence). Ice-melt or the thawing of permafrost involve thresholds (state changes) that allow for abrupt, nonlinear responses to ongoing climate warming (high confidence). These characteristics of ocean and cryosphere change pose risks and challenges to adaptation. $\{1.1$, Box 1.1, 1.3 $\}$

Societies will be exposed, and challenged to adapt, to changes in the ocean and cryosphere even if current and future efforts to reduce greenhouse gas emissions keep global warming well below $2^{\circ} \mathrm{C}$ (very high confidence). Ocean and cryosphere-related mitigation and adaptation measures include options that address the causes of climate change, support biological and ecological adaptation, or enhance societal adaptation. Most ocean-based local mitigation and adaptation measures have limited effectiveness to mitigate climate change and reduce its consequences at the global scale, but are useful to implement because they address local risks, often have co-benefits such as biodiversity conservation, and have few adverse side effects. Effective mitigation at a global scale will reduce the need and cost of adaptation, and reduce the risks of surpassing limits to adaptation. Ocean-based carbon dioxide removal at the global scale has potentially large negative ecosystem consequences. $\{1.6 .1,1.6 .2$, Cross-Chapter Box 2 in Chapter 1\}

The scale and cross-boundary dimensions of changes in the ocean and cryosphere challenge the ability of communities, cultures and nations to respond effectively within existing governance frameworks (high confidence). Profound economic and institutional transformations are needed if climate-resilient development is to be achieved (high confidence). Changes in the ocean and cryosphere, the ecosystem services that they provide, the drivers of those changes, and the risks to marine, coastal, polar and mountain ecosystems, occur on spatial and temporal scales that may not align within existing governance structures and practices (medium confidence). This report highlights the requirements for transformative governance, international and transboundary cooperation, and greater empowerment of local communities in the governance of the ocean, coasts, and cryosphere in a changing climate. $\{1.5,1.7$, Cross-Chapter Box 2 in Chapter 1, Cross-Chapter Box 3 in Chapter 1\}
Robust assessments of ocean and cryosphere change, and the development of context-specific governance and response options, depend on utilising and strengthening all available knowledge systems (high confidence). Scientific knowledge from observations, models and syntheses provides global to local scale understandings of climate change (very high confidence). Indigenous knowledge (IK) and local knowledge (LK) provide context-specific and socio-culturally relevant understandings for effective responses and policies (medium confidence). Education and climate literacy enable climate action and adaptation (high confidence). $\{1.8$, Cross-Chapter Box 4 in Chapter 1$\}$

Long-term sustained observations and continued modelling are critical for detecting, understanding and predicting ocean and cryosphere change, providing the knowledge to inform risk assessments and adaptation planning (high confidence). Knowledge gaps exist in scientific knowledge for important regions, parameters and processes of ocean and cryosphere change, including for physically plausible, high impact changes like high end sea level rise scenarios that would be costly if realised without effective adaptation planning and even then may exceed limits to adaptation. Means such as expert judgement, scenario building, and invoking multiple lines of evidence enable comprehensive risk assessments even in cases of uncertain future ocean and cryosphere changes. \{1.8.1, 1.9.2; Cross-Chapter Box 5 in Chapter 1$\}$ 


\subsection{Why this Special Report?}

All people depend directly or indirectly on the ocean and cryosphere (see FAQ1.1). Coasts are the most densely populated areas on Earth. As of $2010,28 \%$ of the global population (1.9 billion people) were living in areas less than $100 \mathrm{~km}$ from the coastline and less than $100 \mathrm{~m}$ above sea level, including 17 major cities which are each home to more than 5 million people (Kummu et al., 2016). Small Island Developing States are together home to around 65 million people (UN, 2015a). The low elevation coastal zone (land less than $10 \mathrm{~m}$ above sea level), where people and infrastructure are most exposed to coastal hazards, is currently home to around $11 \%$ of the global population (around 680 million people), and by 2050 the population in this zone is projected to grow to more than one billion under all Shared Socioeconomic Pathways (SSPs) (Section 4.3.3.2; Merkens et al., 2016; O'Neill et al., 2017). In 2010, approximately 4 million people lived in the Arctic (Section 3.5.1), and an increase of only $4 \%$ is projected for 2030 (Heleniak, 2014) compared to $16-23 \%$ for the global population increase (O'Neill et al., 2017). Almost 10\% of the global population (around 670 million people) lived in high mountain regions in 2010, and by 2050 the population in these regions is expected to grow to between 736-844 million across the SSPs (Section 2.1). For people living in close contact with the ocean and cryosphere, these systems provide essential livelihoods, food security, well-being and cultural identity, but are also a source of hazards (Sections 1.5.1, 1.5.2).

Even people living far from the ocean or cryosphere depend on these systems. Snow and glacier melt from high mountains helps to sustain the rivers that deliver water resources to downstream populations (Kaser et al., 2010; Sharma et al., 2019). In the Indus and Ganges river basins, for example, snow and glacier melt provides enough water to grow food crops to sustain a balanced diet for 38 million people, and supports the livelihoods of 129 million farmers (Biemans et al., 2019). The ocean and cryosphere regulate global climate and weather; the ocean is the primary source of rain and snowfall needed to sustain life on land, and uptake of heat and carbon into the ocean has so far limited the magnitude of anthropogenic warming experienced at the Earth's surface (Section 1.2). The ocean's biosphere is responsible for about half of the primary production on Earth, and around 17\% of the non-grain protein in human diets is derived from the ocean (FAO, 2018). Communities far from the coast can also be exposed to changes in the ocean through extreme weather events. Ocean and cryosphere changes can result in differing consequences and benefits on local to global scales; for example, declining sea ice in the Arctic is allowing access to shorter international shipping routes but restricting traditional sea ice based travel for Arctic communities.

Human activities are estimated to have so far caused approximately $1^{\circ} \mathrm{C}$ of global warming $\left(0.8^{\circ} \mathrm{C}-1.2^{\circ} \mathrm{C}\right.$ likely range; above pre-industrial levels; IPCC, 2018). The IPCC Fifth Assessment Report (AR5) concluded that, 'Warming of the climate system is unequivocal, and since the 1950s, many of the observed changes are unprecedented over decades to millennia. The atmosphere and ocean have warmed, the amounts of snow and ice have diminished, sea level has risen, and the concentrations of greenhouse gases have increased' (IPCC, 2013). Subsequently, Parties to the Paris Agreement aimed to strengthen the global response to the threats of climate change, including by 'holding the increase in global average temperature to well below $2^{\circ} \mathrm{C}$ above pre-industrial levels and pursuing efforts to limit the temperature increase to $1.5^{\circ} \mathrm{C}^{\prime}$ (UNFCCC, 2015).

Pervasive ocean and cryosphere changes that are already being caused by human-induced climate change are observed from high mountains, to the polar regions, to coasts and into the deep reaches of the ocean. Changes by the end of this century are expected to be larger under high greenhouse gas emission futures compared with low-emission futures (Cross-Chapter Box 1 in Chapter 1), and inaction on reducing emissions will have large economic costs. If human impacts on the ocean continue unabated, declines in ocean health and services are projected to cost the global economy 428 billion USD $\mathrm{yr}^{-1}$ by 2050, and 1.979 trillion USD $\mathrm{yr}^{-1}$ by 2100 . Alternatively, steps to reduce these impacts could save more than a trillion dollars USD $\mathrm{yr}^{-1}$ by 2100 (Ackerman, 2013). Similarly, sea level rise scenarios of 25 to $123 \mathrm{~cm}$ by 2100 without adaptation are expected to see $0.2-4.6 \%$ of the global population impacted by coastal flooding annually, with average annual losses amounting to $0.3-9.3 \%$ of global GDP. Investment in adaptation reduces by 2 to 3 orders of magnitude the number of people flooded and the losses caused (Hinkel et al., 2014).

The United Nations 2030 SDGs (UN, 2015b) are all connected to varying extents with the ocean and cryosphere (see FAQ1.2). Climate action (SDG 13) would limit future ocean and cryosphere changes (high confidence; Cross-Chapter Box 1 in Chapter 1, Figure 1.5, Chapter 2 to 6), and would reduce risks to SDGs that are fundamentally linked to the ocean and cryosphere, including life below water, and clean water and sanitation. (Sections 2.4, 4.4, 5.4; Szabo et al., 2016; LeBlanc et al., 2017; Singh et al., 2018; Visbeck, 2018; Wymann von Dach et al., 2018; Kulonen, Accepted). Other goals for sustainable development depend on the services the ocean and cryosphere provide or are impacted by ocean and cryosphere change; including, life on land, health and wellbeing, eradicating poverty and hunger, economic growth, clean energy, infrastructure, and sustainable cities and communities. Progress on the other SDGs (education, gender equality, reduced inequalities, responsible consumption, strong institutions, and partnerships for the goals) are important for reducing the vulnerability of people and communities to the risks of ocean and cryosphere changes (Section 1.5; 2.3), and for supporting mitigation and adaptation responses (Sections 1.6, 1.7 and 1.8.3; medium confidence).

The characteristics of ocean and cryosphere change (Section 1.3) present particular challenges to climate-resilient development pathways (CRDPs). Ocean acidification and deoxygenation, ice sheet and glacier mass loss, and permafrost degradation are expected to be irreversible on time scales relevant to human societies and ecosystems (Lenton et al., 2008; Solomon et al., 2009; Frölicher and Joos, 2010; Cai et al., 2016; Kopp et al., 2016). Ocean and cryosphere changes also have the potential to worsen anthropogenic climate change, globally and regionally; for example, by additional greenhouse gas emissions released through permafrost thaw that would intensify anthropogenic climate change globally, or by increasing the absorption of solar radiation through snow and ice loss in the Arctic 
that is causing regional climate to warm at more than twice the global rate (AMAP, 2017; Steffen et al., 2018). Ocean and cryosphere changes place particular pressures on the adaptive capacities of cultures who maintain centuries to millennia-old relationships to the planet's polar, mountain, and coastal environments, as well as on cities, states and nations whose territorial boundaries are being transformed by ongoing sea level rise (Gerrard and Wannier, 2013). The scale and cross-boundary dimensions of changes in the ocean and cryosphere challenge the ability of current local, regional and international governance structures to respond (Section 1.7). Profound economic and institutional transformations are needed if climate-resilient development is to be achieved, including ambitious mitigation efforts to avoid the risks of large-scale and abrupt ocean and cryosphere changes.

The commissioning of this IPCC special report recognises the interconnected ways in which the ocean and cryosphere are expected to change in a warming climate. SROCC assesses new knowledge since AR5 and provides an integrated approach across IPCC working groups I and II, linking physical changes with their ecological and human impacts, and the strategies to respond and adapt to future risks. It is one of three special reports being produced by the IPCC during its Sixth Assessment Cycle (in addition to the three working groups' main assessment reports). The concurrent IPCC Special Report on Climate Change and Land (released August 2019) links to SROCC where terrestrial environments and their habitability interact closely with the ocean or cryosphere, such as in mountain, Arctic, and coastal regions. SR15 concluded that human-induced warming will reach $1.5^{\circ} \mathrm{C}$ between $2030-2052$ if it continues to increase at the current rate (high confidence), and that there are widespread benefits to human and natural systems of limiting warming to $1.5^{\circ} \mathrm{C}$ compared with $2^{\circ} \mathrm{C}$ or more (high confidence; IPCC, 2018).

\title{
Box 1.1 Major Components and Characteristics of the Ocean and Cryosphere
}

\author{
Ocean \\ The global ocean is the interconnected body of saline water that encompasses polar to equatorial climate zones and covers $71 \%$ of the \\ Earth surface. It includes the Arctic, Pacific, Atlantic, Indian and Southern Oceans, as well as their marginal seas. The ocean contains \\ about $97 \%$ of the Earth's water, supplies $99 \%$ of the Earth's biologically habitable space, and provides roughly half of the primary \\ production on Earth.
}

Coasts are where ocean and land processes interact, and includes coastal cities, deltas, estuaries, and other coastal ecosystems such as mangrove forests. Low elevation coastal zones (less than $10 \mathrm{~m}$ above sea level) are densely populated and particularly exposed to hazards from the ocean (Chapters 4 to 6, Cross-Chapter Box 9). Moving into the ocean, the continental shelf represents the shallow ocean areas (depth $<200 \mathrm{~m}$ ) that surround continents and islands, before the seafloor descends at the continental slope into the deep ocean. The edge of the continental shelf is often used to identify the coastal ocean from the open ocean. Ocean depth and distance from the coast may influence the governance and economic access that applies to ocean areas (Cross-Chapter Box 3 in Chapter 1).

The average depth of the global ocean is about 3,700 m, with a maximum depth of more than $10,000 \mathrm{~m}$. The ocean is vertically stratified with less dense water sitting above more dense layers, determined by the seawater temperature, salinity and pressure. The surface of the ocean is in direct contact with the atmosphere, except for sea ice covered regions. Sunlight penetrates the water column and supports primary production (by phytoplankton) down to 50-200 m depth (epipelagic zone). Atmospheric-driven mixing occurs from the sea surface and into the mesopelagic zone (200-1,000 m). The distinction between the upper ocean and deep ocean depends on the processes being considered.

The ocean is a fundamental climate regulator on seasonal to millennial time scales. Seawater has a heat capacity four times larger than air and holds vast quantities of dissolved carbon. Heat, water, and biogeochemically relevant gases (e.g., $\mathrm{O}_{2}$ and $\mathrm{CO}_{2}$ ) exchange at the air-sea interface, and ocean currents and mixing caused by winds, tides, wave dynamics, density differences and turbulence redistribute these throughout the global ocean (Box 1.1, Figure 1).

\section{Cryosphere}

The cryosphere refers to frozen components of the Earth system that are at or below the land and ocean surface. These include snow, glaciers, ice sheets, ice shelves, icebergs, sea ice, lake ice, river ice, permafrost and seasonally frozen ground. Cryosphere is widespread in polar regions (Chapter 3) and high mountains (Chapter 2), and changes in the cryosphere can have far-reaching and even global impacts (Chapters 2 to 6, Cross-Chapter Box 9).

Snow is common in polar and mountain regions. It can ultimately either melt seasonally or transform into ice layers that build glaciers and ice sheets. Snow feeds groundwater and river runoff together with glacier melt causes natural hazards (avalanches, rain-on-snow flood events) and is a critical economic resource for hydropower and tourism. Snow plays a major role in maintaining high mountain and Arctic ecosystems, affects the Earth's energy budget by reflecting solar radiation (albedo effect), and influences the temperature of underlying permafrost. 


\section{Box 1.1 (continued)}

Ice sheets and glaciers are land-based ice, built up by accumulating snowfall on their surface. Presently, around 10\% of Earth's land area is covered by glaciers or ice sheets, which in total hold about $69 \%$ of Earth's freshwater (Gleick, 1996). Ice sheets and glaciers flow, and at their margins ice and/or melt water is discharged into lakes, rivers or the ocean. The largest ice bodies on Earth are the Greenland and Antarctic ice sheets. Marine-based sections of ice sheets (e.g., West Antarctic lce Sheet) sit upon bedrock that largely lies below sea level and are in contact with ocean heat, making them vulnerable to rapid and irreversible ice loss. Ice sheets and glaciers that lose more ice than they accumulate contribute to global sea level rise.

Ice shelves are extensions of ice sheets and glaciers that float in the surrounding ocean. The transition between the grounded part of an ice sheet and a floating ice shelf is called the grounding line. Changes in ice shelf size do not directly contribute to sea level rise, but buttressing of ice shelves restrict the flow of land-based ice past the grounding line into the ocean.

Sea ice forms from freezing of seawater, and sea ice on the ocean surface is further thickened by snow accumulation. Sea ice may be discontinuous pieces moved on the ocean surface by wind and currents (pack ice), or a motionless sheet attached to the coast or to ice shelves (fast ice). Sea ice provides many critical functions: it provides essential habitat for polar species and supports the livelihoods of people in the Arctic (including Indigenous peoples); regulates climate by reflecting solar radiation; inhibits ocean-atmosphere exchange of heat, momentum and gases (including $\mathrm{CO}_{2}$ ); supports global deep ocean circulation via dense (cold and salty) water formation; and aids or hinders transportation and travel routes in the polar regions.

Permafrost is ground (soil or rock containing ice and frozen organic material) that remains at or below $0^{\circ} \mathrm{C}$ for at least two consecutive years. It occurs on land in polar and high mountain areas, and also as submarine permafrost in shallow parts of the Arctic and Southern Oceans. Permafrost thickness ranges from less than $1 \mathrm{~m}$ to greater than 1,000 m. It usually occurs beneath an active layer, which thaws and freezes annually. Unlike glaciers and snow, the spatial distribution and temporal changes of permafrost cannot easily be observed. Permafrost thaw can cause hazards, including ground subsidence or landslides, and influence global climate through emissions of greenhouse gases from microbial breakdown of previously frozen organic carbon.

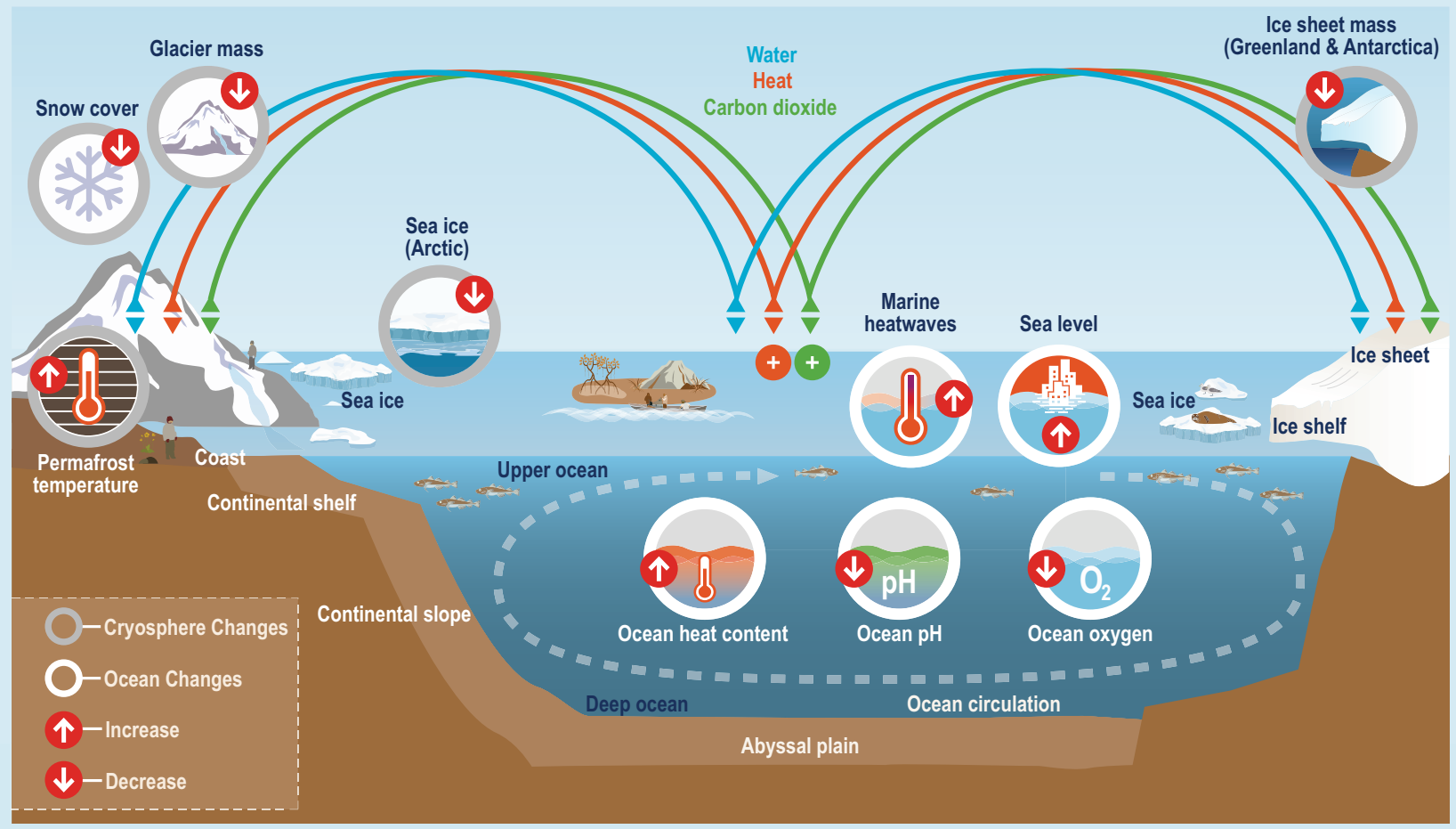

Box 1.1, Figure 1 | Schematic illustration of key components and changes of the ocean and cryosphere, and their linkages in the Earth system through the movement of heat, water, and carbon dioxide (Section 1.2). Climate change-related effects in the ocean include sea level rise, increasing ocean heat content and marine heat waves, ocean deoxygenation, and ocean acidification (Section 1.4.1). Changes in the cryosphere include the decline of Arctic sea ice extent, Antarctic and Greenland ice sheet mass loss, glacier mass loss, permafrost thaw and decreasing snow cover extent (Section 1.4.2). For illustration purposes, a few examples of where humans directly interact with ocean and cryosphere are shown. 


\subsection{Role of the Ocean and Cryosphere in the Earth System}

\subsubsection{Ocean and Cryosphere in Earth's Energy, Water and Biogeochemical Cycles}

The ocean and cryosphere play a key role in the Earth system. Powered by the Sun's energy, large quantities of energy, water and biogeochemical elements (predominantly carbon, nitrogen, oxygen and hydrogen) are exchanged between all components of the Earth system, including between the ocean and cryosphere (Box 1.1, Figure 1).

During an equilibrium (stable) climate state, the amount of incoming solar energy is balanced by an equal amount of outgoing radiation at the top of Earth's atmosphere (Hansen et al., 2011). At the Earth's surface energy from the Sun is transformed into various forms (heat, potential, latent, kinetic, and chemical), that drive weather systems in the atmosphere and currents in the ocean, fuel photosynthesis on land and in the ocean, and fundamentally determine the climate (Trenberth et al., 2014). The ocean has a large capacity to store and release heat, and the Earth's energy budget can be effectively monitored through the heat content of the ocean on time scales longer than one year (Palmer and McNeall, 2014; von Schuckmann et al., 2016; Cheng et al., 2018). The large heat capacity of the ocean leads to different characteristics of the ocean response to external forcings compared with the atmosphere (Sections 1.3, 1.4). The reflective properties of snow and ice also play an important role in regulating climate via the albedo effect. Increased amounts of solar energy are absorbed when snow or ice are replaced by less reflective land or ocean surfaces, resulting in a climate change feedback responsible for amplified changes.

Water is exchanged between the ocean, atmosphere, land and cryosphere as part of the hydrological cycle driven by solar heating (Box 1.1, Figure 1; Trenberth et al., 2007; Lagerloef et al., 2010; Durack et al., 2016). Evaporation from the surface ocean is the main source of water in the atmosphere, which is moved back to the Earth's surface as precipitation (Gimeno et al., 2012). The hydrological cycle is closed by the eventual return of water to the ocean by rivers, streams, and groundwater flow, and through ice discharge and melting of ice sheets and glaciers $(Y u, 2018)$. Hydrological extremes related to the ocean include floods from extreme rainfall (including tropical cyclones) or ocean circulation-related droughts (Sections 6.3, 6.5), while cryosphere-related flooding can be caused by rapid snow melt and melt water discharge events (Sections 2.3, 3.4).

Ninety-two percent of the carbon on Earth that is not locked up in geological reservoirs (e.g., in sedimentary rocks or coal, oil and gas reservoirs) resides in the ocean (Sarmiento and Gruber, 2002). Most of this is in the form of dissolved inorganic carbon, some of which readily exchanges with $\mathrm{CO}_{2}$ in the overlying atmosphere. This represents a major control on atmospheric $\mathrm{CO}_{2}$ and makes the ocean and its carbon cycle one of the most important climate regulators in the Earth system, especially on time scales of a few hundred years and more (Sigman and Boyle, 2000; Berner and Kothavala, 2001). The ocean also contains as much organic carbon (mostly in the form of dissolved organic matter) as the total vegetation on land (Jiao et al., 2010; Hansell, 2013). Primary production in the ocean, which is as large as that on land (Field et al., 1998), fuels complex food-webs that provide essential food for people.

Ocean circulation and mixing redistribute heat and carbon over large distances and depths (Delworth et al., 2017). The ocean moves heat laterally from the tropics towards polar regions (Rhines et al., 2008). Vertical redistribution of heat and carbon occurs where warm, low-density surface ocean waters transform into cool high-density waters that sink to deeper layers of the ocean (Talley, 2013), taking high carbon concentrations with them (Gruber et al., 2019). Driven by winds, ocean circulation also brings cold water up from deep layers (upwelling) in some regions, allowing heat, oxygen and carbon exchange between the deep ocean and the atmosphere (Oschlies et al., 2018; Shi et al., 2018) and fuelling biological production (Sarmiento and Gruber, 2006).

\subsubsection{Interactions Between the Ocean and Cryosphere}

The ocean and cryosphere are interconnected in a multitude of ways (Box 1.1, Figure 1). Evaporation from the ocean provides snowfall that builds and sustains the ice sheets and glaciers that store large amounts of frozen water on land (Section 4.2.1). The vast ice sheets in Antarctica and Greenland currently hold about $66 \mathrm{~m}$ of potential global sea level rise (Fretwell et al., 2013), although the loss of a large fraction of this potential would require millennia of ice sheet retreat. Ocean temperature and sea level affect ice sheet, glacier and ice shelf stability in places where the base of ice bodies are in direct contact with ocean water (Section 3.3.1). The nonlinear response of ice-melt to ocean temperature changes means that even slight increases in ocean temperature have the potential to rapidly melt and destabilise large sections of an ice sheet or ice shelf (Section 3.3.1.5).

The formation of sea ice leads to the production of dense ocean water that contributes to the deep ocean circulation (Section 3.3.3.2). Paleoclimate evidence and modelling indicates that releases of large amounts of glacier and ice sheet melt water into the surface ocean can disrupt deep overturning circulation of the ocean, causing global climate impacts (Knutti et al., 2004; Golledge et al., 2019). Ice sheet melt water in the Antarctic may cause changes in surface ocean salinity, stratification and circulation, that feedback to generate further ocean-driven melting of marine-based ice sheets (Golledge et al., 2019) and promote sea ice formation (Purich et al., 2018). The cryosphere and ocean further link through the movement of biogeochemical nutrients. For example, iron accumulated in sea ice during winter is released to the ocean during the spring and summer melt, helping to fuel ocean productivity in the seasonal sea ice zone (Tagliabue et al., 2017). Nutrient rich sediments delivered by glaciers further connect cryosphere processes to ocean productivity (Arrigo et al., 2017). 


\subsection{Time Scales, Thresholds and Detection of Ocean and Cryosphere Change}

It takes hundreds of years to millennia for the entire deep ocean to turn over (Matsumoto, 2007; Gebbie and Huybers, 2012), while renewal of the large ice sheets requires many thousands of years (Huybrechts and de Wolde, 1999). Long response times mean that the deep ocean and the large ice sheets tend to lag behind in their response to the rapidly changing climate at Earth's surface, and that they will continue to change even after radiative forcing stabilises (e.g., Golledge et al., 2015; Figure 1.1a). Such 'committed' changes mean that some ocean and cryosphere changes are essentially irreversible on time scales relevant to human societies (decades to centuries), even in the presence of immediate action to limit further global warming (e.g., Section 4.2.3.5).

While some aspects of the ocean and cryosphere might respond in a linear (i.e., directly proportional) manner to a perturbation by some external forcing, this may change fundamentally when critical thresholds are reached. A very important example for such a threshold is the transition from frozen water to liquid water at around $0^{\circ} \mathrm{C}$ that can lead to rapid acceleration of ice-melt or permafrost thaw (e.g., Abram et al., 2013; Trusel et al., 2018). Such thresholds often act as tipping points, as they are associated with rapid and abrupt changes even when the underlying forcing changes gradually (Figure 1.1a, 1.1c). Tipping elements include, for example, the collapse of the ocean's large-scale overturning circulation in the Atlantic (Section 6.7), or the collapse of the West Antarctic Ice Sheet though a process called marine ice sheet instability (Cross-Chapter Box 8 in Chapter 3; Lenton et al., 2008). Potential ocean and cryosphere tipping elements form part of the scientific case for efforts to limit climate warming to well below $2^{\circ} \mathrm{C}$ (IPCC, 2018).

Anthropogenically forced change occurs against a backdrop of substantial natural variability (Figure 1.1b). The anthropogenic signal is already detectable in global surface air temperature and several other climate variables, including ocean temperature and salinity (IPCC, 2014), but short observational records and large year-toyear variability mean that formal detection is not yet the case for many expected ocean and cryosphere changes (Jones et al., 2016). 'Time of Emergence' refers to the time when anthropogenic change signals emerge from the background noise of natural variability in a pre-defined reference period Hawkins and Sutton, 2012; (Figure 1.1b; Section 5.2, Box 5.1). For some variables, (e.g., for those associated with ocean acidification), the current signals emerge from this natural variability within a few decades, whereas for others, such as primary production and expected Antarctic-wide sea ice decline, the signal may not emerge for many more decades even under high emission scenarios (Collins et al., 2013; Keller et al., 2014; Rodgers et al., 2015; Frölicher et al., 2016; Jones et al., 2016).
'Detection and Attribution' assesses evidence for past changes in the ocean and cryosphere, relative to normal/reference-interval conditions (detection), and the extent to which these changes have been caused by anthropogenic climate change or by other factors (attribution) (Bindoff et al., 2013; Cramer et al., 2014; Knutson et al., 2017; Figure 1.1d). Reliable detection and attribution is fundamental to our understanding of the scientific basis of climate change (Hegerl et al., 2010). For example, the main attribution conclusion of the IPCC 4th Assessment Report (AR4), in other words, that 'most of the observed increase in global average temperatures since the mid-20th century is very likely due to the observed increase in anthropogenic greenhouse gas concentrations', has had a strong impact on climate policy (Petersen, 2011). In AR5 this attribution statement was elevated to 'extremely likely' (Bindoff et al., 2013). Statistical approaches for attribution often involve using contrasting forcing scenarios in climate model experiments to detect the forcing that best explains an observed change (Figure 1.1d). In addition to passing the statistical test, a successful attribution also requires a firm process understanding. Confident attribution remains challenging though, especially when there are multiple or confounding factors that influence the state of a system (Hegerl et al., 2010). Particular challenges to detection and attribution in the ocean and cryosphere include the often short observational records (Section 1.8.1.1, Figure 1.3), which are particularly confounding given the long adjustment time scales to anthropogenic forcing of many properties of interest.

Extreme climate events (e.g., marine heatwaves or storm surges) push a system to near or beyond the ends of its normally observed range (Seneviratne et al., 2012; Figure 1.1b; Chapter 6;). Extremes can be very costly in terms of loss of life, ecosystem destruction, and economic damage. In a system affected by climate change, the recurrence and intensity of these extreme events can change much faster and have greater impacts than changes of the average system state (Easterling et al., 2000; Parmesan et al., 2000; Hughes et al., 2018). Of particular concern are 'compound events', when the joint probability of two or more properties of a system is extreme at the same time or closely connected in time and space (Cross-Chapter Box 5 in Chapter 1; Sections 4.3.4, 6.8). Such a compound event is given, for example, when marine heatwaves co-occur with very low nutrient levels in the ocean potentially resulting in extreme impacts (Bond et al., 2015). The interconnectedness of the ocean and cryosphere (Section 1.2.2) can also lead to cascading effects where changes in one element trigger secondary changes in completely different but connected elements of the systems, including its socioeconomic aspects. (Figure 1.1e). An example is the large change in ocean productivity triggered by the changes in circulation and iron inputs induced by the large outflow of melt waters from Greenland (Kanna et al., 2018). New methodologies for attributing extreme events and the risks they bring to climate change have emerged since AR5 (Trenberth et al., 2015; Stott et al., 2016; Kirchmeier-Young et al., 2017; Otto, 2017), especially also for the attribution of individual events through an assessment of the fraction of attributable risk (Figure 1.1f). 
(a) Dynamic Response of Systems

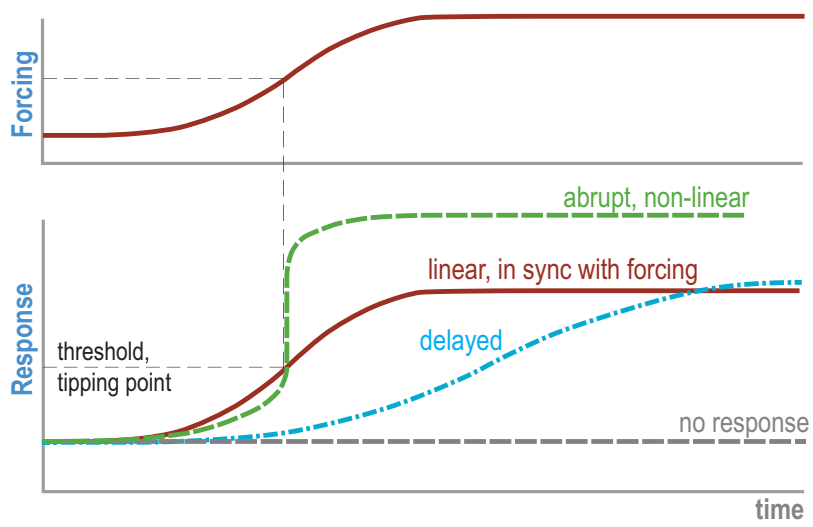

(c) Tipping Points

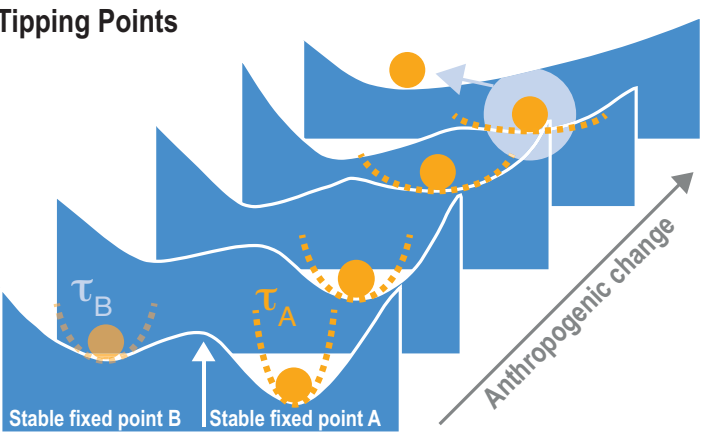

Tipping point

$(\tau)=$ system's response time

(e) Cascading Effects

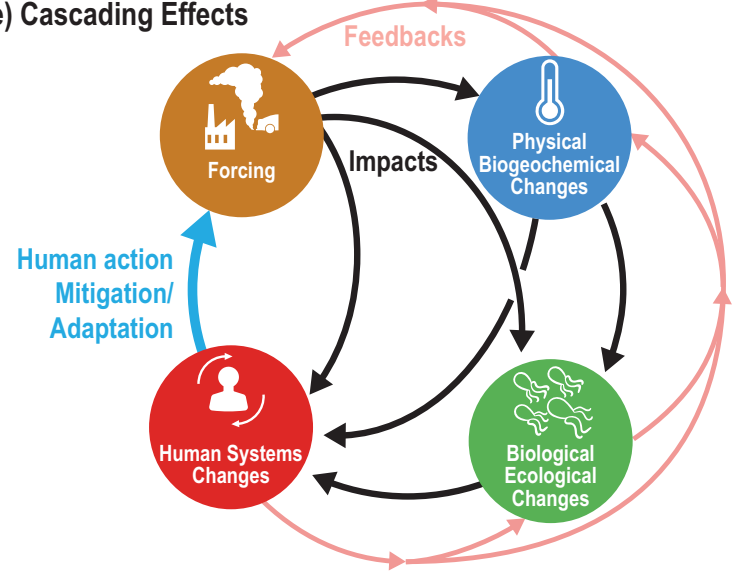

(b) Variability, Time of Emergence and Extremes

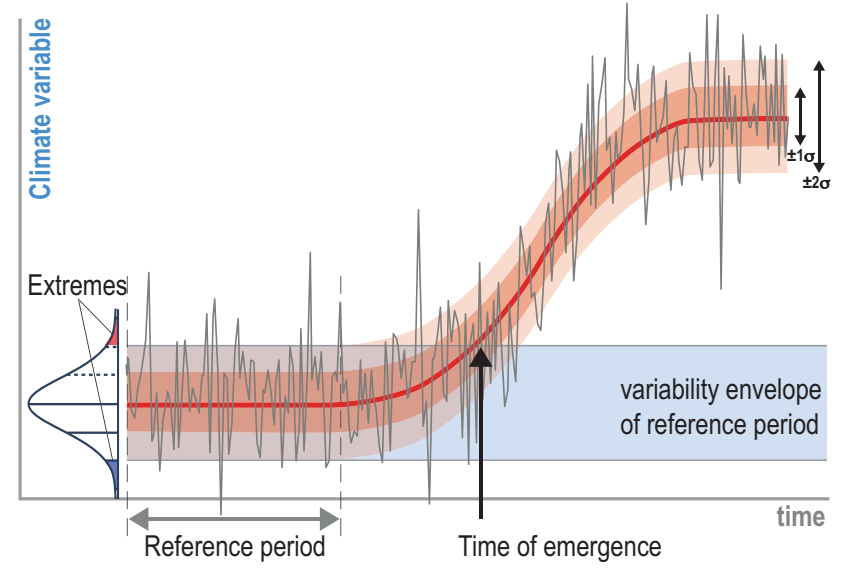

(d) Detection and Attribution

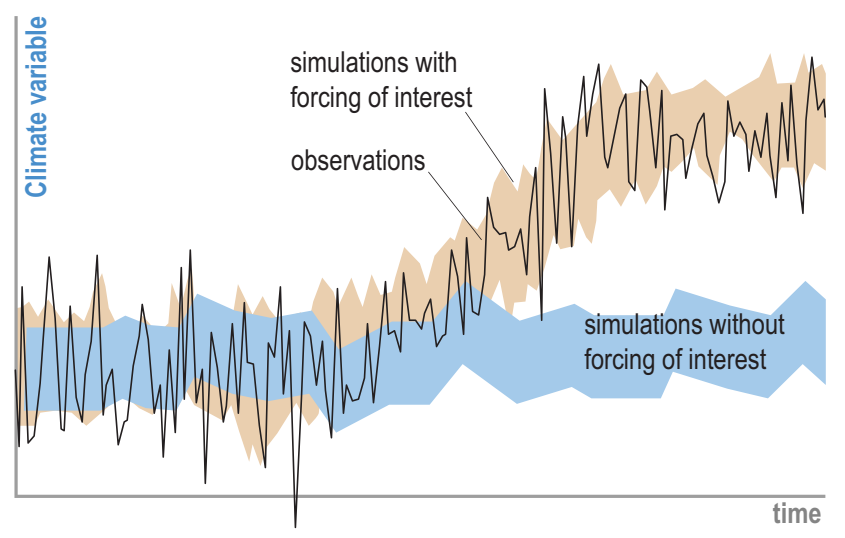

\section{(f) Event Attribution}

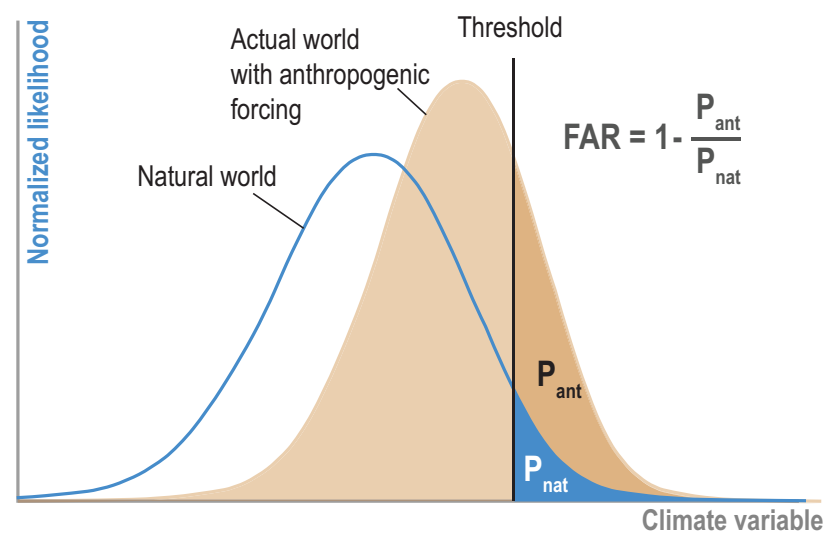

Figure 1.1 Schematic of key concepts associated with changes in the ocean and cryosphere. (a) Differing responses of systems to gradual forcing (e.g., linear, delayed, abrupt, nonlinear). (b) Evolution of a dynamical system in time, revealing both natural (unforced) variability and a response to a new (e.g., anthropogenic) forcing. Key concepts include (i) the time of emergence and (ii) extreme events near or beyond the observed range of variability. (c) Tipping points and the change of their behaviour through time in response to, for example, anthropogenic change (adapted from Lenton et al., 2008). The two minima represent two stable fixed points, separated by a maximum representing an unstable fixed point, acting as a tipping point. The ball represents the state of the system with the red dash line indicating the stability of the fixed point and the system's response time to small perturbations. (d) Detection and attribution, i.e., the statistical framework used to determine whether a change occurs or not (detection), and whether this detected change is caused by a particular set of forcings (e.g., greenhouse gases) (attribution). (e) Cascading effects, where changes in one part of a system inevitably affect the state in another, and so forth, ultimately affecting the state of the entire system. These cascading effects can also trigger feedbacks, altering the forcing. (f) Event attribution and fraction of attributable risk. The blue (orange) probability density function shows the likelihood of the occurrence of a particular value of a climate variable of interest under natural (present = including anthropogenic forcing) conditions. The corresponding areas above the threshold indicate the probabilities $P_{n a t}$ and $P_{\text {ant }}$ of exceedance of this threshold. The fraction of attributable risk (given by $F A R=1-P_{\text {ant }} / P_{\text {nat }}$ ) indicates the likelihood that a particular event has occurred as a consequence of anthropogenic change (adapted from Stott et al., 2016). 


\subsection{Changes in the Ocean and Cryosphere}

Earth's climate, ocean and cryosphere vary across a wide range of time scales. This includes the seasonal growth and melting of sea ice, interannual variation of ocean temperature caused by the El Niño-Southern Oscillation and ice age cycles across tens to hundreds of thousands of years.

Climate variability can arise from internally generated (i.e., unforced) fluctuations in the climate system. Variability can also occur in response to external forcings, including volcanic eruptions, changes in the Earth's orbit around the Sun, oscillations in solar activity and changing atmospheric greenhouse gas concentrations.

Since the onset of the industrial revolution, human activities have had a strong impact on the climate system, including the ocean and cryosphere. Human activities have altered the external forcings acting on Earth's climate (Myhre et al., 2013) by changes in land use (albedo), and changes in atmospheric aerosols (e.g., soot) from the burning of biomass and fossil fuels. Most significantly, human activities have led to an accumulation of greenhouse gases (including $\mathrm{CO}_{2}$ ) in the atmosphere as a result of the burning of fossil fuels, cement production, agriculture and land use change. In 2016, the global average atmospheric $\mathrm{CO}_{2}$ concentration crossed 400 parts per million, a level Earth's atmosphere did not experience for at least the past 800,000 years and possibly much longer (Lüthi et al., 2008; Fischer et al., 2018). These anthropogenic forcings have not only warmed the ocean and begun to melt the cryosphere, but have also led to widespread biogeochemical changes driven by the oceanic uptake of anthropogenic $\mathrm{CO}_{2}$ from the atmosphere (IPCC, 2013).

It is now nearly three decades since the first assessment report of the IPCC, and over that time evidence and confidence in observed and projected ocean and cryosphere changes have grown (very high confidence; Table SM1.1). Confidence in climate warming and its anthropogenic causes has increased across assessment cycles; robust detection was not yet possible in 1990, but has been characterised as unequivocal since AR4 in 2007. Projections of near-term warming rates in early reports have been realised over the subsequent decades, while projections have tended to err on the side of caution for sea level rise and ocean heat uptake that have developed faster than predicted (Brysse et al., 2013; Section 4.2, 5.2). Areas of concern in early reports which were expected but not observable are now emerging. The expected acceleration of sea level rise is now observed with high confidence (Section 4.2). There is emerging evidence in sustained observations and from long-term palaeoclimate reconstructions for the expected slow-down of AMOC (medium confidence), although this remains to be properly attributed (Section 6.7). Significant sea level rise contributions from Antarctic ice sheet mass loss (very high confidence), which earlier reports did not expect to manifest this century, are already being observed (Section 3.3.1). Other newly emergent characteristics of ocean and cryosphere change (e.g., marine heat waves; Section 6.4) are assessed for the first time in SROCC.
AR5 (IPCC, 2013; IPCC, 2014) provides ample evidence of profound and pervasive changes in the ocean and cryosphere (Sections 1.4.1, 1.4.2), and along with the recent SR15 report (IPCC, 2018), is the point of departure for the updated assessments made in SROCC.

\subsubsection{Observed and Projected Changes in the Ocean}

Increasing greenhouse gases in the atmosphere cause heat uptake in the Earth system (Section 1.2) and as reported since 1970, there is high confidence ${ }^{3}$ that the majority (more than $90 \%$ ) of the extra thermal energy in the Earth's system is stored in the global ocean (IPCC, 2013). Mean ocean surface temperature has increased since the 1970 s at a rate of $0.11(0.09-0.13)^{\circ} \mathrm{C}$ per decade (high confidence), and forms part of a long-term warming of the surface ocean since the mid-19th century. The upper ocean (0-700 m, virtually certain) and intermediate ocean (700 to 2,000 m, likely) have warmed since the 1970s. Ocean heat uptake has continued unabated since AR5 (Sections 3.2.1.2.1, 5.2), increasing the risk of marine heat waves and other extreme events (Section 6.4). During the 21st century, ocean warming is projected to continue even if anthropogenic greenhouse gas emissions cease (Sections 1.3, 5.2). The global water cycle has been altered, resulting in substantial regional changes in sea surface salinity (high confidence; Rhein et al., 2013), which is expected to continue in the future (Sections 5.2.2, 6.3, 6.5).

The rate of sea level rise since the mid-19th century has been larger than the mean rate of the previous two millennia (high confidence). Over the period 1901 to 2010, global mean sea level rose by 0.19 (0.17-0.21) m (high confidence) (Church et al., 2013; Table SM1.1). Sea level rise continues due to freshwater added to the ocean by melting of glaciers and ice sheets, and as a result of ocean expansion due to continuous ocean warming, with a projected acceleration and century to millennial-scale commitments for ongoing rise (Section 4.2.3). In SROCC, recent developments of ice sheet modelling are assessed (Sections 1.8, 4.3, Cross-Chapter Box 8 in Chapter 3) and the projected sea level rise at the end of 21 st century is higher than reported in AR5 but with a larger uncertainty range (Sections 4.2.3.2, 4.2.3.3).

By 2011 , the ocean had taken up about $30 \pm 7 \%$ of the anthropogenic $\mathrm{CO}_{2}$ that had been released to the atmosphere since the industrial revolution (Ciais et al., 2013; Section 5.2). In response, ocean pH decreased by 0.1 since the beginning of the industrial era (high confidence), corresponding to an increase in acidity of $26 \%$ (Table SM1.1) and leading to both positive and negative biological and ecological impacts (high confidence) (Gattuso et al., 2014). Evidence is increasing that the ocean's oxygen content is declining (Oschlies et al., 2018). AR5 did not come to a final conclusion with regard to potential long-term changes in ocean productivity due to short observational records and divergent scientific evidence (Boyd et al., 2014; Section 5.2.2). Ocean acidification and deoxygenation are projected to continue over the next century with high confidence (Sections 3.2.2.3, 5.2.2).

3 Confidence/likelihood statements in Sections 1.4.1 and 1.4.2 derived from AR5 and SR 15, unless otherwise specified. 


\subsubsection{Observed and Projected Changes in the Cryosphere}

Changes in the cryosphere documented in AR5 included the widespread retreat of glaciers (high confidence), mass loss from the Greenland and Antarctic ice sheets (high confidence) and declining extents of Arctic sea ice (very high confidence) and Northern Hemisphere spring snow cover (very high confidence; IPCC, 2013; Vaughan et al., 2013).

A particularly rapid change in Earth's cryosphere has been the decrease in Arctic sea ice extent in all seasons (Section 3.2.1.1). AR5 assessed that there was medium confidence that a nearly ice-free summer Arctic Ocean is likely to occur before mid-century under a high emissions future (IPCC, 2013), and SR15 assessed that ice-free summers are projected to occur at least once per century at $1.5^{\circ} \mathrm{C}$ of warming, and at least once per decade at $2{ }^{\circ} \mathrm{C}$ of warming above pre-industrial levels (IPCC, 2018). Sea ice thickness is decreasing further in the Northern Hemisphere and older ice that has survived multiple summers is rapidly disappearing; most sea ice in the Arctic is now 'first year' ice that grows in the autumn and winter but melts during the spring and summer (AMAP, 2017).

AR5 assessed that the annual mean loss from the Greenland ice sheet very likely substantially increased from $34(-6-74) \mathrm{Gt}_{\mathrm{yr}}{ }^{-1}$ (billion tonnes $\mathrm{yr}^{-1}$ ) over the period 1992-2001, to 215 (157-274) Gt yr ${ }^{-1}$ over the period 2002-2011 (IPCC, 2013). The average rate of ice loss from the Antarctic ice sheet also likely increased from 30 (-37-97) Gt $\mathrm{yr}^{-1}$ over the period 1992-2001, to 147 (72-221) Gt yr ${ }^{-1}$ over the period 2002-2011 (IPCC, 2013). The average rate of ice loss from glaciers around the world (excluding glaciers on the periphery of the ice sheets), was very likely 226 (91-361) Gt yr ${ }^{-1}$ over the period 19712009, and 275 (140-410) Gt yr-1 over the period 1993-2009 (IPCC, 2013). The Greenland and Antarctic ice sheets are continuing to lose mass at an accelerating rate (Section 3.3) and glaciers are continuing to lose mass worldwide (Section 2.2.3, Cross-Chapter Box 6 in Chapter 2). Confidence in the quantification of glacier and ice sheet mass loss has increased across successive IPCC reports (Table SM1.1) due to the development of remote sensing observational methods (Section 1.8.1).

Changes in seasonal snow are best documented for the Northern Hemisphere. AR5 reported that the extent of snow cover has decreased since the mid-20th century (very high confidence). Negative trends in both snow depth and duration are also detected with station observations (medium confidence), although results depend on elevation and observational period (Section 2.2.2). AR5 assessed that permafrost temperatures have increased in most regions since the early 1980s (high confidence), and the rate of increase has varied regionally (IPCC, 2013). Methane and carbon dioxide release from soil organic carbon is projected to continue in high mountain and polar regions (Box 2.2), and SROCC has used multiple lines of evidence to reduce uncertainty in permafrost change assessments (Cross-Chapter Box 5 in Chapter 1, Section 3.4.3.1.1).

\section{Cross-Chapter Box 1 | Scenarios, Pathways and Reference Periods}

Authors: Nerilie Abram (Australia), William Cheung (Canada), Lijing Cheng (China), Thomas Frölicher (Switzerland), Mathias Hauser (Switzerland), Shengping He (Norway/China), Anne Hollowed (USA), Ben Marzeion (Germany), Samuel Morin (France), Anna Pirani (Italy), Didier Swingedouw (France)

\section{Introduction}

Assessing the future risks and opportunities that climate change will bring for the ocean and cryosphere, and for their dependent ecosystems and human communities, is a main objective of this report. However, the future is inherently uncertain. A well-established methodological approach that the Special Report on the Ocean and Cryosphere in a Changing Climate (SROCC) report uses to assess the future under these uncertainties is through scenario analysis (Kainuma et al., 2018). The ultimate physical driver of the ocean and cryosphere changes that SROCC assesses are greenhouse gas emissions, while the exposure to hazards and the future risks to natural and human systems are also shaped social, economic and governance factors (Cross-Chapter Box 2 in Chapter 1; Section 1.5). This Cross-Chapter Box introduces the main scenarios that are used in the SROCC assessment. Examples of key climate change indicators in the atmosphere and ocean projected under future greenhouse gas emission scenarios are also provided (Table CB1.1).

\section{Scenarios and pathways}

Scenarios are a plausible description of how the future may develop based on a coherent and internally consistent set of assumptions about key driving forces and relationships. Pathways refer to the temporal evolution of natural and/or human systems towards a future state. In SROCC, assessments of future change frequently use climate model projections forced by pathways of future radiative forcing changes related to different socioeconomic scenarios.

Representative Concentration Pathways (RCPs) are a set of time series of plausible future concentrations of greenhouse gases, aerosols and chemically active gases, as well as land use changes (Moss et al., 2008; Moss et al., 2010; van Vuuren et al., 2011a; Figure SM1.1). The word representative signifies that each RCP provides only one of many possible pathways that would lead to the specific radiative forcing characteristics. The term pathway emphasises the fact that not only the long-term concentration levels, but also the trajectory taken over time to reach that outcome are of interest. 
Four RCPs were used for projections of the future climate in the Coupled Model Intercomparison Project Phase 5 (CMIP5) (Taylor et al., 2012). They are identified by their approximate anthropogenic radiative forcing (in $\mathrm{W} \mathrm{m}^{-2}$, relative to 1750) by 2100: RCP2.6, RCP4.5, RCP6.0, and RCP8.5 (Figure SM1.1). RCP8.5 is a high greenhouse gas emission scenario without effective climate change mitigation policies, leading to continued and sustained growth in atmospheric greenhouse gas concentrations (Riahi et al., 2011). RCP2.6 represents a low greenhouse gas emission, high mitigation future that gives a two in three chance of limiting global atmospheric surface warming to below $2^{\circ} \mathrm{C}$ by the end of the century (van Vuuren et al., 2011b; Collins et al., 2013; Allen et al., 2018). Achieving the RCP2.6 pathway would require implementation of negative emissions technologies at a not-yet-proven scale to remove greenhouse gases from the air, in addition to other mitigation strategies such as energy from sustainable sources and existing nature-based strategies (Gasser et al., 2015; Sanderson et al., 2016; Royal Society, 2018; National Academies of Sciences, 2019). An even more stringent RCP1.9 pathway is considered most compatible with limiting global warming to below $1.5^{\circ} \mathrm{C}$, called a $1.5^{\circ} \mathrm{C}$-consistent pathway in the Special Report on Global Warming of $1.5^{\circ} \mathrm{C}$ (SR15; 0'Neill et al., 2016; IPCC, 2018), and will be assessed in the IPCC 6th Assessment Report (AR6) using projections of Phase 6 of the Coupled Model Intercomparison Project (CMIP6). Global fossil $\mathrm{CO}_{2}$ emissions rose more than $2 \%$ in 2018 and $1.6 \%$ in 2017, after a temporary slowdown in emissions from 2014-2016. Current emissions continue to grow in line with the RCP8.5 trajectory (Peters et al., 2012; Le Quéré et al., 2018).

In SROCC, the CMIP5 simulations forced with RCPs are used extensively to assess future ocean and cryosphere changes. In particular, RCP2.6 and RCP8.5 are used to contrast the possible outcomes of low-emission versus high-emission futures, respectively (Table CB1.1). In some cases the SROCC assessments use literature that is based on the earlier Special Report on Emission Scenarios (SRES; IPCC, 2000), and details of these and their approximate RCP equivalents are provided in Tables SM1.3 and SM1.4.

Shared Socioeconomic Pathways (SSPs) complement the RCPs with varying socioeconomic challenges to adaptation and mitigation (e.g., population, economic growth, education, urbanisation and the rate of technological development; O'Neill et al., 2017). The SSPS describe five alternative socioeconomic futures comprising: sustainable development (SSP1), middle-of-the-road development (SSP2), regional rivalry (SSP3), inequality (SSP4), and fossil-fuelled development (SSP5; Figure SM1.1; Kriegler et al., 2016; Riahi et al., 2017). The RCPs set plausible pathways for greenhouse gas concentrations and the climate changes that could occur, and the SSPs set the stage on which reductions in emissions will or will not be achieved within the context of the underlying socioeconomic characteristics and shared policy assumptions of that world. The combination of SSP-based socioeconomic scenarios and RCP-based climate projections provides an integrative frame for climate impact and policy analysis. The SSPs will be included in the CMIP6 simulations to be assessed in AR6 (O'Neill et al., 2016). In SROCC, the SSPs are used only for contextualising estimates from the literature on varying future populations in regions exposed to ocean and cryosphere changes.

\section{Baselines and reference intervals}

A baseline provides a reference period from which changes can be evaluated. In the context of anthropogenic climate change, the baseline should ideally approximate the 'pre-industrial' conditions before significant human influences on the climate began. The IPCC 5th Assessment Report (AR5) and SR15 (Allen et al., 2018) use 1850-1900 as the pre-industrial baseline for assessing historical and future climate change. Atmospheric greenhouse gas concentrations and global surface temperatures had already begun to rise in this interval from early industrialisation (Abram et al., 2016; Hawkins et al., 2017; Schurer et al., 2017). However, the scarcity of reliable climate observations represents a major challenge for quantifying earlier pre-industrial states (Hawkins et al., 2017). To maintain consistency across IPCC reports, the 1850-1900 pre-industrial baseline is used wherever possible in SROCC, recognising that this is a compromise between data coverage and representativeness of typical pre-industrial conditions.

In SROCC, the 1986-2005 reference interval used in AR5 is referred to as the recent past, and a 2006-2015 reference is used for present day, consistent with SR15 (Allen et al., 2018). The 2006-2015 reference interval incorporates near-global upper ocean data coverage and reasonably comprehensive remote-sensing cryosphere data (Section 1.8.1), and aligns this report with a more current reference than the 1986-2005 reference adopted by AR5. This 10-year present day period is short relative to natural variability. However, at this decadal scale the bias in the present day interval due to natural variability is generally small compared to differences between present day conditions and the pre-industrial baseline. There is also no indication of global average surface temperature in either 1986-2005 or 2006-2015 being substantially biased by short-term variability (Allen et al., 2018), consistent with the AR5 finding that each of the last three decades has been successively warmer at the Earth's surface than any preceding decade since 1850 (IPCC, 2013).

SROCC commonly provides future change assessments for two key intervals: A near term interval of 2031-2050 is comparable to a single generation time scale from present day, and incorporates the interval when global warming is likely to reach $1.5^{\circ} \mathrm{C}$ if warming continues at the current rate (IPCC, 2018). An end-of-century interval of 2081-2100 represents the average climate 
Table CB1.1 | Projected change in global mean surface air temperature and key ocean variables for the near term (2031-2050) and end-of-century (2081-2100) relative to the recent past (1986-2005) reference period from CMIP5. See Table SM1.2 for the list of CMIP5 models and ensemble member used for calculating these projections. Small differences in the projections given here compared with AR5 (e.g., Table 12.2 in Collins et al., 2013) reflect differences in the number of models available now compared to at the time of the AR5 assessment (Table SM1.2).

\begin{tabular}{|c|c|c|c|c|c|}
\hline & \multirow[b]{2}{*}{ Scenario } & \multicolumn{2}{|c|}{ Near term: 2031-2050 } & \multicolumn{2}{|c|}{ End-of-century: 2081-2100 } \\
\hline & & Mean & $5-95 \%$ range & Mean & $5-95 \%$ range \\
\hline \multirow{4}{*}{$\begin{array}{l}\text { Global Mean Surface } \\
\text { Air temperature }\left({ }^{\circ} \mathrm{C}\right)^{\text {a }}\end{array}$} & $\mathrm{RCP} 2.6$ & 0.9 & 0.5 to 1.4 & 1.0 & 0.3 to 1.7 \\
\hline & RCP4.5 & 1.1 & 0.7 to 1.5 & 1.8 & 1.0 to 2.6 \\
\hline & RCP6.0 & 1.0 & 0.5 to 1.4 & 2.3 & 1.4 to 3.2 \\
\hline & RCP8.5 & 1.4 & 0.9 to 1.8 & 3.7 & 2.6 to 4.8 \\
\hline \multirow{2}{*}{$\begin{array}{l}\text { Global Mean Sea } \\
\text { Surface Temperature }\left({ }^{\circ} \mathrm{C}\right)^{\mathrm{b}} \\
(\text { Section 5.2.5) }\end{array}$} & RCP2.6 & 0.64 & 0.33 to 0.96 & 0.73 & 0.20 to 1.27 \\
\hline & RCP8.5 & 0.95 & 0.60 to 1.29 & 2.58 & 1.64 to 3.51 \\
\hline \multirow{2}{*}{ 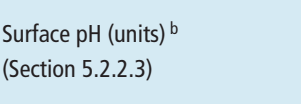 } & RCP2.6 & -0.072 & -0.072 to -0.072 & -0.065 & -0.065 to -0.066 \\
\hline & RCP8.5 & -0.108 & -0.106 to -0.110 & -0.315 & -0.313 to -0.317 \\
\hline \multirow{2}{*}{$\begin{array}{l}\text { Dissolved Oxygen }(100-600 \mathrm{~m}) \\
(\% \text { change) } \\
(\text { Section } 5.2 .2 .4)^{\mathrm{b}}\end{array}$} & $\mathrm{RCP} 2.6$ & -0.9 & -0.3 to -1.5 & -0.6 & 0.0 to -1.2 \\
\hline & RCP8.5 & -1.4 & -1.0 to -1.8 & -3.9 & -2.9 to -5.0 \\
\hline
\end{tabular}

Notes:

${ }^{a}$ Calculated following the same procedure as the IPCC 5th Assessment Report (AR5) (Table 12.2 in Collins et al., 2013). The 5-95\% model range of global mean surface air temperature across CMIP5 projections was assessed in AR5 as the likely range, after accounting for additional uncertainties or different levels of confidence in models.

${ }^{\mathrm{b}}$ The $5-95 \%$ model range for global mean sea surface temperature, surface $\mathrm{pH}$ and dissolved oxygen (100-600 $\mathrm{m}$ ) as referred to in the SROCC assessment as the very likely range (Section 1.9.2, Figure 1.4).

conditions reached at the end of the standard CMIP5 future climate simulations and is relevant to long-term infrastructure planning and climate-resilient development pathways (CRDPs) (Cross-Chapter Box 2 in Chapter 1). In some cases where committed changes exist over multi-century time scales, such as the assessment of future sea level rise (Section 4.3.2) or deep ocean oxygen changes (Section 5.2.4.2, Table 5.5), SROCC also considers model evidence for long-term changes beyond the end of the current century.

\section{Key indicators of future ocean and cryosphere change}

Table CB1.1 compiles information on key indicators of climate change in the atmosphere and ocean. This information is given for different RCPs and for changes in the near term and end-of-century assessment intervals, relative to the recent past, noting that this does not capture changes that have already taken place since the pre-industrial baseline. SR15 assessed that global mean surface warming from the pre-industrial (1850-1900) to the recent past $(1986-2005)$ reference period was $0.63^{\circ} \mathrm{C}$ (likely range of $0.57^{\circ} \mathrm{C}-$ $0.69^{\circ} \mathrm{C}$ ), and during the present day interval $(2006-2015)$ was $0.87^{\circ} \mathrm{C}$ (likely range of $0.75^{\circ} \mathrm{C}-0.99^{\circ} \mathrm{C}$ ) higher than the average over the 1850-1900 pre-industrial period (very high confidence; IPCC, 2018).

These key climate and ocean change indicators allow for some harmonisation of the risk assessments in the chapters of SROCC. Projections of future change across a wider range of ocean and cryosphere components is also provided in Figure 1.5. Ocean and cryosphere changes and risks by the end-of-century (2081-2100) are expected to be larger under high greenhouse gas emission scenarios, compared with low greenhouse gas emission scenarios (very high confidence) (Table CB1.1, Figure 1.5). 


\subsection{Risk and Impacts Related to Ocean and Cryosphere Change}

SROCC assesses the risks (i.e., potential for adverse consequences) and impacts (i.e., manifested risk) resulting from climate-related changes in the ocean and cryosphere. Knowledge on risk is essential for conceiving and implementing adequate responses. Cross-Chapter Box 2 in Chapter 1 introduces key concepts of risk, adaptation, resilience and transformation, and explains why and how they matter for this report.

In SROCC, the term 'natural system' describes the biological and physical components of the environment, independent of human involvement but potentially affected by human activities. 'Natural systems' may refer to portions of the total system without necessarily considering all its components (e.g., an ocean upwelling system). Throughout the assessment usage of 'natural system' does not imply a system unaltered by human activities.

'Human systems' include physiological, health, socio-cultural, belief, technological, economic, food, political, and legal systems, among others. Humans have depended upon the Earth's ocean (WOA, 2016; IPBES, 2018b) and cryosphere (AMAP, 2011; Hovelsrud et al., 2011; Watt-Cloutier, 2018) for many millennia (Redman, 1999). Contemporary human populations still depend directly on elements of the ocean and cryosphere, and the ecosystem services they provide, but at a much larger scale and with greater environmental impact than in pre-industrial times (Inniss and Simcock, 2017).

An ecosystem is a functional unit consisting of living organisms, their non-living environment, and the interactions within and between them. Ecosystems can be nested within other ecosystems and their scale can range from very small to the entire biosphere. Today, most ecosystems either contain humans as key organisms, or are influenced by the effects of human activities in their environment. In SROCC, a social-ecological system describes the combined system and all of its sub-components and refers specifically to the interaction of natural and human systems.

The ocean and cryosphere are unique systems that have intrinsic value, including the ecosystems and biodiversity they support. Frameworks of Ecosystem Services and Nature's Contributions to People are both used within SROCC to assess the impacts of changes in the ocean and cryosphere on humans directly, and through changes to the ecosystems that support human life and civilisations (Sections 2.3, 3.4.3.2, 4.3.3.5, 5.4, 6.4, 6.5, 6.8). The Millennium Ecosystem Assessment (MEA, 2005) established a conceptual Ecosystem Services framework between biodiversity, human well-being, and drivers of change. This framework highlights that natural systems provide vital life-support services to humans and the planet, including direct material services (e.g., food, timber), non-material services (e.g., cultural continuity, health), and many services that regulate environmental status (e.g., soil formation, water purification). This framework supports decision-making by quantifying benefits for valuation and trade-off analyses. The Ecosystem Services framework has been challenged as monetising the relationships of people with nature, and undervaluing small-scale livelihoods, cultural values and other considerations that contribute little to global commerce (Díaz et al., 2018). More recent frameworks, such as Nature's Contributions to People (Díaz et al., 2018), used in the Intergovernmental Platform on Biodiversity and Ecosystem Services assessments (IPBES), aim to better encompass the non-commercial ways that nature contributes to human quality of life.

\section{Cross-Chapter Box 2 | Key Concepts of Risk, Adaptation, Resilience and Transformation}

Authors: Matthias Garschagen (Germany), Carolina Adler (Switzerland/Australia), Susie Crate (USA), Hélène Jacot Des Combes (Fiji/France), Bruce Glavovic (New Zealand/South Africa), Sherilee Harper (Canada), Elisabeth Holland (Fij//USA), Gary Kofinas (USA), Sean O'Donoghue (South Africa), Ben Orlove (USA), Zita Sebesvari (Hungary/Germany), Martin Sommerkorn (Norway/Germany)

This box introduces key concepts used in the Special Report on the Ocean and Cryosphere in a Changing Climate (SROCC) in relation to risk, adaptation, resilience, and transformation. Building on an assessment of the current literature, it provides a conceptual framing for the report and for the assessments within its chapters. Full definitions of key terms are provided in the SROCC Annex I: Glossary.

\section{Risk and adaptation}

SROCC considers risk from climate change-related effects on the ocean and cryosphere as the result of the interaction between: (1) environmental hazards triggered by climate change, (2) exposure of humans, infrastructure and ecosystems to those hazards, and (3) systems' vulnerabilities. Risk refers to the potential for adverse consequences, and impacts refer to materialised effects of climate change. Next to assessing risk and impacts specifically resulting from climate change-related effects on the ocean, coast and cryosphere, SROCC is also concerned with the options to reduce climate-related risk.

Beyond mitigation, adaptation is a key avenue to reduce risk (Section 1.6). Adaptation can also include exploiting new opportunities; however, this box focuses on risk, and thus, the latter is not discussed in detail here. Adaptation efforts link into the causal fabric of risk by reducing existing and future vulnerability, exposure, and/or (where possible) hazards (Figure CB2.1). Addressing the different risk components (hazards, exposure and vulnerability) involves assessing and selecting options for policy. 
Cross-Chapter Box 2 (continued)

\section{Actions to reduce Hazards}

Examples include:

- Ecosystem-based measures to reduce coastal flooding

- Mangroves to alleviate coastal storm energy

-Water reservoirs to buffer low-flows and water scarcity

\section{Limits to Adaptation}

-E.g. physical, ecological, technological, economic, political, institutional, psychological, and/or socio-cultural

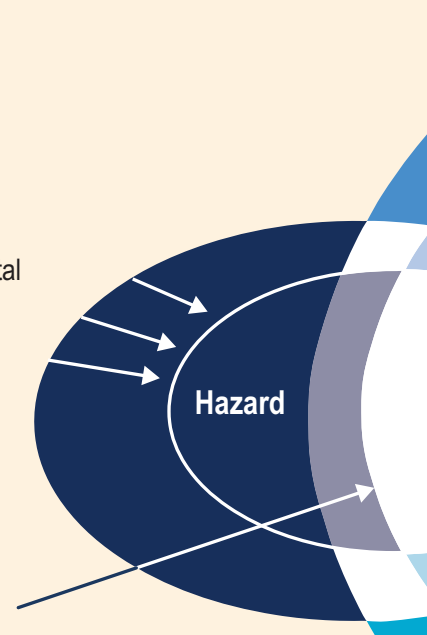

(-)

(-

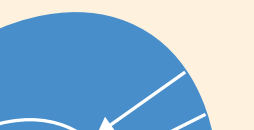

Vulnerability

4
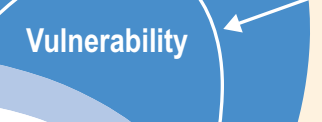

$\rightarrow$

Risk

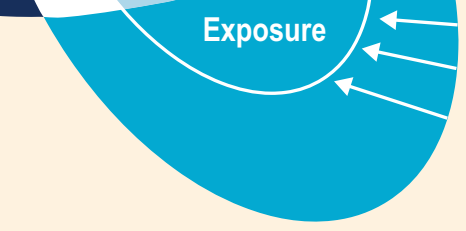

\section{Actions to reduce}

Vulnerability

Examples include:

- Social protection

- Livelihood diversification

- Insurance solutions

- Hazard-proof housing

and infrastructure

\section{Actions to reduce}

Exposure

Examples include:

- Coastal retreat and resettlement

- Risk sensitive land use planning

- Early warning systems and evacuations

Figure CB2.1 | There are options for risk reduction through adaptation. Adaptation can reduce risk by addressing one or more of the three risk factors: vulnerability, exposure, and/or hazard. The reduction of vulnerability, exposure, and/or hazard potential can be achieved through different policy and action choices over time until limits to adaptation might be reached. The figure builds on the conceptual framework of risk used in the IPCC 5th Assessment Report (AR5) (Oppenheimer et al., 2014).

and action. Such decision-making entails evaluation of the effectiveness, efficiency, efficacy and acceptance of actions. Adaptation responses are more effective when they promote resilience to climate change, consider plausible futures and unexpected events, strengthen essential or desired characteristics as well as values of the responding system and/or make adjustments to avoid unsustainable pathways (high agreement, medium evidence; Section 2.3; Box 2.4; 4.4.4; 4.4.5).

Adaptation requires adaptive capacity, which for human systems includes assets (financial, physical, and/or ecological), capital (social and institutional), knowledge and technical know-how (Klein et al., 2014). The extent of adaptive capacity determines adaptation potential, but does not necessarily translate into effective adaptation if awareness of the need to act, the willingness to act and/or the cooperation needed to act is lacking (high confidence; Sections 2.3; Box 2.4; 4.3.2.6.3; 5.5.2.4).

There are limits to adaptation, which include, for example, physical, ecological, technological, economic, political, institutional, psychological and/or socio-cultural aspects (medium evidence, high agreement) (Dow et al., 2013; Barnett et al., 2014; Klein et al., 2014). For example, the ability to adapt to sea level rise depends, in part, on the elevation of the low-lying islands and coasts in question, but also on the capacity to successfully negotiate protection or relocation measures socially and politically (Cross-Chapter Box 9, also see Section 6.4.3 for a wider overview). Limits to adaptation are sometimes considered as something different from barriers to adaptation. Barriers can in principle be overcome if adaptive capacity is available (e.g., where funding is made available), even though overcoming barriers is often hard in reality, particularly for resource-poor communities and countries (high confidence; Section 4.4.3). Limits to adaptation are reached when adaptation no longer allows an actor or ecosystem to secure valued objectives or key functions from intolerable risks (Section 4.4.2; Dow et al., 2013). Defining tolerable risks and key system functions is, therefore, of central importance for the assessment of limits to adaptation.

Residual risks (i.e., the risk that endures following adaptation and risk reduction efforts) remain even where adaptation is possible (very high confidence; Chapters 2-6; Section 6.3.2; Table 6.2). Residual risks have bearing on the emerging debate about loss and damage (Huq et al., 2013; Warner and van der Geest, 2013; Boyd et al., 2017; Djalante et al., 2018; Mechler et al., 2018; Roy et al., 2018). This report addresses loss and damage in relation to slow onset processes, including ocean changes (Section 5.4.2.3), sea level rise (Section 4.3), and glacier retreat (Section 2.3.6), and polar cryosphere changes (Section 3.4.3.3.4), as well as rapid onset hazards such as tropical cyclones (Chapter 6). The assessment encompasses non-economic losses, including the impacts on intrinsic and 


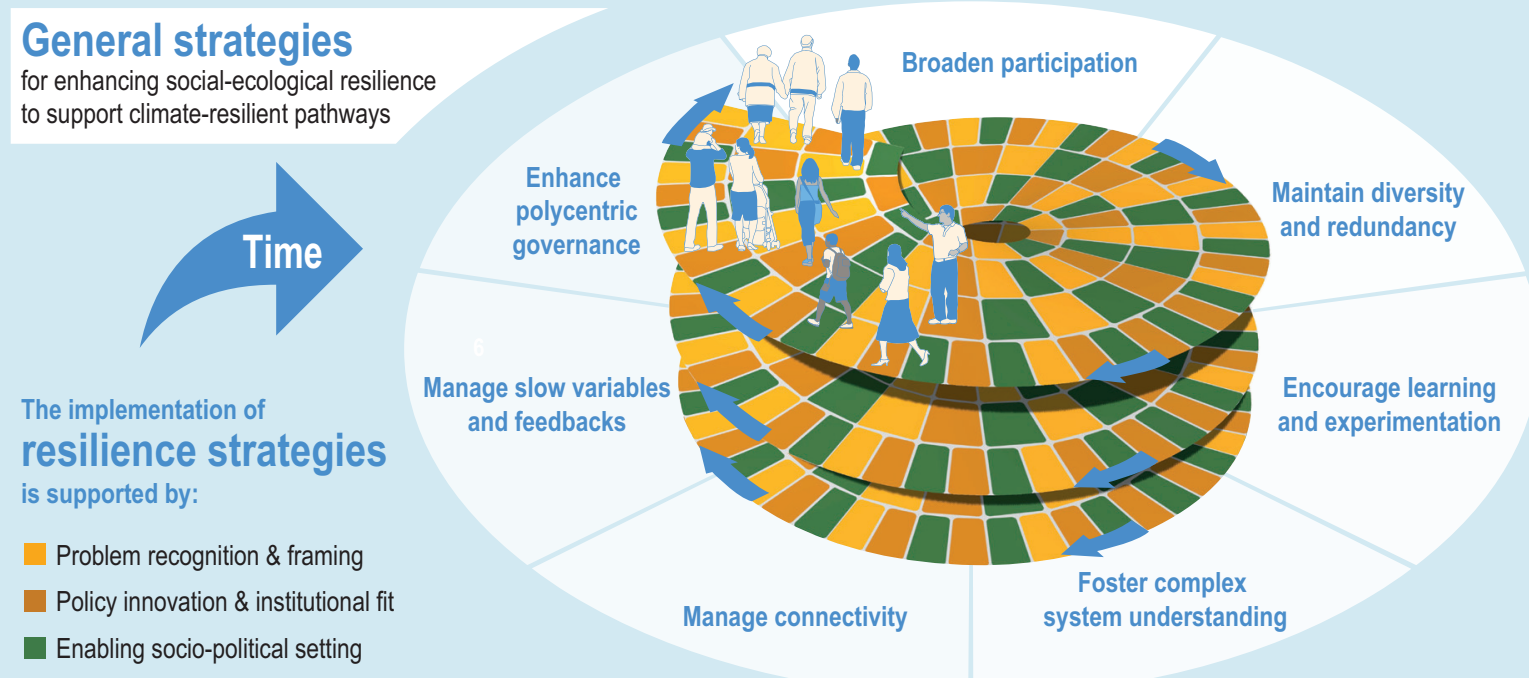

Figure CB2.2 | General strategies for enhancing social-ecological resilience to support climate-resilient pathways have been identified. The seven strategies are adapted from synthesis papers by Biggs et al. (2012) and Quinlan et al. (2016), the illustration of the climate-resilient development pathway (CRDP) builds on Figure SPM9 in the IPCC 5th Assessment Report (AR5) (IPCC, 2014).

spiritual attributes with which high mountain societies value their landscapes (Section 2.3.5); the interconnected relationship with, and reliance upon, the land, water and ice for culture, livelihoods and wellbeing in the Arctic (Section 3.4.3.3); and cultural heritage and displacement addressed in the Cross-Chapter Box on low-lying islands and coasts (Cross-Chapter Box 9; Burkett, 2016; Markham et al., 2016; Tschakert et al., 2017; Huggel et al., 2018).

\section{Building resilience}

Addressing climate change-related risk, impacts (including extreme events and shocks) and trade-offs together with shaping the trajectories of social and ecological systems is facilitated by considering resilience (Biggs et al., 2012; Quinlan et al., 2016). In SROCC, resilience is understood as the capacity of interconnected social, economic and ecological systems to cope with disturbances by reorganising in ways that maintain their essential function, structure, and identity (Walker et al., 2004). Resilience may be considered as a positive attribute of a system and an aspirational goal when it contributes to the capacity for adaptation and learning without changing the structure, function, and identity of the system (Walker et al., 2004; Steiner, 2015). Alternately, resilience may be used descriptively as a system property that is neither good nor bad (Walker et al., 2004; Chapin et al., 2009; Weichselgartner and Kelman, 2014). For example, a system can be highly resilient in keeping its unfavoured attributes, such as poverty or institutional rigidity (Carpenter and Brock, 2008). Critics of the resilience concept warn that the application of resilience to social systems is problematic when the responsibility for resilience building is shifted onto the shoulders of vulnerable and resource-poor populations (e.g., Chandler, 2013; Reid, 2013; Rigg and Oven, 2015; Tierney, 2015; Olsson et al., 2017).

Applying the concept of resilience in mitigation and adaptation planning builds the capacity of a social-ecological system to navigate anticipated changes and unexpected events (Biggs et al., 2012; Varma et al., 2014; Sud et al., 2015). Resilience also emphasises social-ecological system dynamics, including the possibility of crossing critical thresholds and experiencing a regime shift (i.e., state change). Seven general strategies for building social-ecological resilience have been identified (Figure CB2.2; Ostrom, 2010; Biggs et al., 2012; Quinlan et al., 2016). The concept of resilience also allows analysts, accessors of risk and decision makers to recognise how climate-change related risks often cannot be fully avoided or alleviated despite adaptation. For SROCC, this is especially relevant along low-lying coasts, high mountain areas and the polar regions (medium evidence, high agreement; Sections 2.3; 2.4; 3.5, 6.8, 6.9).

Many efforts are underway to apply resilience thinking in assessments, management practices, policy making and the day-today practices of affected sectors and local communities. For example, leaders of the Pacific small island developing states use the Framework for Resilient Development in the Pacific, which integrates climate change and disaster risk management (Pacific Community, 2016; Cross-Chapter Box 9). In the Philippines, a new framework has been developed to conduct full inventories of 
Cross-Chapter Box 2 (continued)

actual and projected loss and damage due to climate change and associated disasters such as from cyclones. Creating such an inventory is difficult due to the disconnect between tools for climate change assessment and those for post disaster assessment (Florano, 2018). In Arctic Alaska, evaluative frameworks are being applied to determine needs, responsibilities, and alternative actions associated with coastal village relocations (Bronen, 2015; Cross-Chapter Box 9). In all these initiatives, resilience is a key consideration for enabling CRDPs.

Climate-resilient development pathways (CRDPs)

CRDPs are a relatively new concept to describe climate change mitigation and adaptation trajectories that strengthen sustainable development and efforts to eradicate poverty and reduce inequalities while promoting fair and cross-scalar adaptation to, and resilience in, a changing climate (Kainuma et al., 2018; Roy et al., 2018). CRDPs are increasingly being explored as an approach for combining scientific assessments, stakeholder participation, and forward-looking development planning, acknowledging that pursuing CRDP is not only a technical challenge of risk management but also a social and political process (Roy et al., 2018). Adaptive decision-making over time is key to CRDPs (Haasnoot et al., 2013; Wise et al., 2014; Fazey et al., 2016; Ramm et al., 2017; Bloemen et al., 2018; Lawrence et al., 2018). CRDPs accommodate both the interacting cultural, social, and ecosystem factors that influence multi-stakeholder decision making processes, and the overall sustainability of adaptation measures.

Adequate climate change mitigation and adaptation allows for opportunities for sustainable development pathways and the options for resilience building. CRDPs involve series of mitigation and adaptation choices over time, balancing short-term and long-term goals and accommodating newly available knowledge (Denton et al., 2014). The CRDPs approach has been successfully used, for example, in urban, remote and disadvantaged communities, and can showcase the potential to counter maladaptive choices (e.g., Barnett et al., 2014; Butler et al., 2014; Maru et al., 2014). CRDPs aim to establish narratives of hope and opportunity that can extend beyond risk reduction and coping (Amundsen et al., 2018). Although climate change impacts on the ocean and cryosphere elicit many emotions, including fear, anger, despair and apathy (Cunsolo Willox et al., 2013; Cunsolo and Landman, 2017; Cunsolo and Ellis, 2018), narratives of hope are critical in provoking motivation, creative thinking and behavioural changes in response to climate change (Myers et al., 2012; Smith and Leiserowitz, 2014; Feldman and Hart, 2016; Feldman and Hart, 2018; Prescott and Logan, 2018; Section 1.8.3).

Much of the adaptation and resilience literature published since AR5 highlights the need for transformations that enable effective climate change mitigation (most notably, to decarbonise the economy) (Riahi et al., 2017), and support adaptation (e.g., Pelling et al., 2015; Few et al., 2017). Transformation becomes particularly relevant when existing mitigation and adaptation practices cannot reduce risks and impacts to an acceptable level. Transformative adaptation, therefore, involves fundamental modifications of policies, policy making processes, institutions, human behaviour and cultural values (Pelling et al., 2015; Solecki et al., 2017). Successful transformation requires attention to conditions that allow for such changes, including timing (e.g., windows of opportunity), social readiness (e.g., some level of willingness) and resources to act (e.g., trust, human skill and financial resources; Kofinas et al., 2013; Moore et al., 2014). Examples related to SROCC include shifting from a paradigm of protection reliant on seawalls to living with saltwater as a response to coastal flooding in rural areas (Renaud et al., 2015) or involving fundamental risk management changes in coastal megacities, including retreat (Solecki et al., 2017). Transformation in changing ocean and cryosphere contexts can be fostered by transdisciplinary collaboration between actors in science, government, the private sector, civil society and affected communities (Padmanabhan, 2017; Cross-Chapter Box 3 in Chapter 1; Cross-Chapter Box 4 in Chapter 1).

\subsubsection{Hazards and Opportunities for Natural Systems, Ecosystems, and Human Systems}

Hazards faced by marine and coastal organisms, and the ecosystem services they provide are generally dependent on future greenhouse gas emission pathways, with moderate likelihood under a low-emission future, but high to very high likelihood under higher emission scenarios (very high confidence) (Mora et al., 2013; Gattuso et al., 2015). Hazards to marine ecosystems assessed in AR5 (IPCC, 2014) included degradation of coral reefs (high confidence), ocean deoxygenation (medium confidence) and ocean acidification (high confidence). Shifts in the ranges of plankton and fish were identified with high confidence regionally, but with uncertain trends globally.
SROCC provides more evidence for global shifts in the distribution of marine organisms, and in how the phenology of animals is responding to ocean change (Sections 3.2.3, 5.2). The signature of climate change is now detected in almost all marine ecosystems. Similar trends of changing habitat due to climate change are reported for the cryosphere (Sections 2.2, 3.4.3.2). The risk of irreversible loss of many marine and coastal ecosystems increases with global warming, especially at $2^{\circ} \mathrm{C}$ or more (high confidence; IPCC, 2018). Risk also increases for habitat displacements, both poleward (Section 3.2.4) and to greater ocean depths (Section 5.2.4), or habitat reductions, such as that caused by glacier retreat (Section 2.2.3). 
Changes in the ocean and cryosphere bring hazards that affect the health, wellbeing, safety and security of populations in coastal, mountain and polar environments (Section 2.3.5, 3.4.3, 4.3.2). Some impacts are direct, such as sea level rise or coastal erosion that can displace coastal residents $(4.3 .2 .3,4.4 .2 .6$, Box 4.1). Other effects are indirect; for example, rising ocean temperatures have led to increases in maximum wind speed and rainfall rates in tropical cyclones (Section 6.3), creating hazards with severe consequences for natural and human systems (Sections 4.3, 6.2, 6.3, 6.8). The multiple category 4 and 5 Atlantic hurricanes in 2017 caused the loss of over 3300 lives and more than 350 billion USD in economic damages (Cross-Chapter Box 9; Andrade et al., 2018; Murakami et al., 2018; NOAA, 2018). In mountain regions, glacial lake outburst floods have caused severe impacts on lives, livelihoods and infrastructure that often extend beyond the directly affected areas (Section 2.3.2 and 6.2.2). Some hazards related to ocean and cryosphere change involve abrupt and irreversible changes (Section 1.3), which generate sometimes unpredictable risks, and multiple hazards can coincide to greatly elevate the total risk (Section 6.8.2). For example, combinations of thawing permafrost, sea level rise, loss of sea ice, ocean surface waves and extreme weather events (Thomson and Rogers, 2014; Ford et al., 2017) have damaged Arctic infrastructure (e.g., buildings, roads) (AMAP, 2015; AMAP, 2017), impacted reindeer husbandry livelihoods for Sami and other Arctic Indigenous peoples and impeded access to hunting grounds, other communities and travel routes fundamental to the livelihoods, food security and wellbeing of Inuit and other Northern cultures (Section 3.4.3). In some Arctic regions, tipping points may have already been reached such that adaptive practices can no longer work (Section 3.5).

Climate change impacts on the ocean and cryosphere can also present opportunities, in at least the near- and medium-term. For example, in Nepal warming of high mountain environments and accelerated melting of snow and ice have extended the growing season and crop yields in some regions (Section 2.3; Gaire et al., 2015; Merrey et al., 2018), while tourism and shipping has increased in the Arctic with loss of sea ice (Section 3.2.4). Moreover, rising ocean temperatures redistribute the global fish population, allowing new fishing opportunities while reducing some established fisheries (Bell et al., 2011; Fenichel et al., 2016; Section 5.4). To gain from new opportunities, while also avoiding or mitigating new or increasing hazards, it is necessary to be aware of trade-offs between risks and benefits to understand who is and is not benefiting. For example, opportunities can involve trade-offs with mitigation and/or SDGs (Section 3.5.2), and the balance of economic costs and benefits may differ substantially between the near-term and long-term future (Section 5.4.2.2).

\subsubsection{Exposure of Natural Systems, Ecosystems, and Human Systems}

Exposure to hazards in cryosphere systems occur in the immediate vicinity of cryosphere components, and at regional to global scales where cryosphere changes link to other natural systems. For example, decreasing Arctic sea ice increases exposure for organisms that depend upon habitats provided by sea ice, but also has far-reaching impacts through the resulting direct albedo feedback and amplification of Arctic climate warming (e.g., Pistone et al., 2014) that then locally increases surface melting of the Greenland ice sheet (Liu et al., 2016; Stroeve et al., 2017). Additionally, ice loss from ice sheets contribute to the global-scale exposure of sea level rise, and more local-scale modifications and losses of coastal habitats and ecosystems (Sections 3.2.3 and 4.3.3.5). Interactions within and between natural systems also influence the spatial reach of risks associated with cryosphere change. Permafrost degradation, for example, interacts with ecosystems and climate on various spatial and temporal scales, and feedbacks from these interactions range from local impacts on topography, hydrology and biology, to global-scale impacts via biogeochemical cycling (e.g., methane release) on climate (Sections 2.2, 2.3, 3.4; Kokelj et al., 2015; Grosse et al., 2016).

Exposure to climate change risk exists for virtually all coastal organisms, habitats and ecosystems (Section 5.2), through processes such as inundation and salinisation (Section 4.3), ocean acidification and deoxygenation (Sections 3.2.3, 5.2.3), increasing marine heatwaves (Section 6.4.1.2), and increases in harmful algal blooms and invasive species (Glibert et al., 2014; Gobler et al., 2017; Townhill et al., 2017; Box 5.3). Aggregate impacts of multiple drivers are dramatically altering ecosystem structure and function in the coastal and open ocean (Boyd et al., 2015; Deutsch et al., 2015; Przeslawski et al., 2015), such as coral reefs under increasing pressure from both rising ocean temperature and acidification (Section 5.3.4). Increasing exposure to climate change hazards in open ocean natural systems includes ocean acidification (O'Neill et al., 2017; Section 5.2.3), changes in ocean ventilation, deoxygenation (Shepherd et al., 2017; Breitburg et al., 2018; Section 5.2.2.4), increased cyclone and flood risk (Section 6.3.3) and an increase in extreme El Niño and La Niña events (Section. 6.5.1). Heat content is rapidly increasing within the ocean (Section 5.2.2) and marine heat waves are becoming more frequent across the world ocean (Section 6.4.1).

People who live close to the ocean and/or cryosphere, or depend directly on their resources for livelihoods, are particularly exposed to climate change impacts and hazards (very high confidence) (Barange et al., 2014; Romero-Lankao et al., 2014; AMAP, 2015). These exposures can result in infrastructure damage and failure (Sections 2.3.1.3, 3.4.3, 3.5., 4.3.2), loss of habitability (Sections 2.3.7, 3.4.3, $3.5,4.3 .3)$, changes in air quality (Section 6.5.2), proliferation of disease vectors (Sections 3.4.3.2.2, 5.4.2.1.1), increased morbidity and mortality due to injury, infectious disease, heat stress, and mental health and wellness challenges (Section 3.4.3.3), compromised food and water security (Sections 2.3.1, 3.4.3.3, 4.3.3.6, 5.4.2.1, 6.8.4), degradation of ecosystem services (Sections 2.3.1.2, 2.3.3.4, 4.3.3, 5.4.1, 6.4.2.3), economic and non-economic impacts due to reduced production and social network system disruption (Section 2.3.7), conflict (Sections 2.3.1.14, 3.5) and widespread human migration (Sections 2.3.7, 4.4.3.5; Oppenheimer et al., 2014; van Ruijven et al., 2014; AMAP, 2015; Cunsolo and Ellis, 2018).

This report documents how people residing in coastal and cryosphere regions are already exposed to climate change hazards, and which of these hazards are projected to increase in the future. For example, mountain communities have been exposed to increased rockfall, rock 
avalanches and landslides due to permafrost degradation and glacier shrinkage, and to changes in snow avalanche type and seasonal timing (Section 2.3.1). Cryosphere changes that can impact water availability in mountain regions and for downstream populations (Sections 2.3.1, 2.3.4, 2.3.5) have implications for drinking water, irrigation, livestock grazing, hydropower production and tourism (Section 2.3). Some declining mountain glaciers hold sacred and symbolic meanings for local communities who will experience spiritual losses (Section 2.3.4, 2.3.5, and 2.3.6). Exposures to extreme warming, and continued sea ice and permafrost loss in the Arctic, challenge Indigenous communities with close interdependent relationships of economy, lifestyles, cultural identity, self-sufficiency, Indigenous knowledge, health and wellbeing with the Arctic cryosphere (Section 3.4.3, 3.5).

The population living in low elevation coastal zones (land less than $10 \mathrm{~m}$ above sea level) is projected to increase to more than one billion by 2050 (Section 4.3.2.2). These people and communities are particularly exposed to future sea level rise, rising ocean temperature (including marine heat waves; Section 6.4), enhanced coastal erosion, increasing wind, wave height, storm intensity and ocean acidification (Section 4.3.4). These exposures bring associated risks for livelihoods linked to fisheries, tourism and trade, as well as loss of life, damaged assets, and disruption of basic services including safe water supplies, sanitation, energy and transportation networks (Chapters 4, 5, and 6; Cross-Chapter Box 9).

\subsubsection{Vulnerabilities in Natural Systems, Ecosystems, and Human Systems}

Direct and indirectrisks to natural systems are influenced by vulnerability to climate change as well as deterioration of ecosystem services. For example, about half of species assessed on the northeast United States continental shelf exhibited high to very high climate vulnerability due to temperature preferences and changes in habitat space (Hare et al., 2016), with corresponding northward range shifts for many species (Kleisner et al., 2017) and increased vulnerability for organisms or ecosystems unable to migrate or evolve at the rate required to adapt to ocean and cryosphere changes (Miller et al., 2018). Non-climatic pressures also magnify the vulnerability of ocean and cryosphere ecosystems to climate-related changes, such as overfishing, coastal development, and pollution, including plastic pollution (Halpern et al., 2008; Halpern et al., 2015; IPBES, 2018a; IPBES, 2018b; IPBES, 2018c; IPBES, 2018d). Conventional (fossil fuel-based) plastics produced in 2015 accounted for $3.8 \%$ of global $\mathrm{CO}_{2}$ emissions and could reach up to $15 \%$ by 2050 (Zheng and Suh, 2019).

The vulnerability of mountain, Arctic and coastal communities is affected by social, political, historical, cultural, economic, institutional, environmental, geographical and/or demographic factors such as gender, age, race, class, caste, Indigeneity and disability (Thomas et al., 2019; Sections 2.3.6 and 3.5; Cross-Chapter Box 9). Disparities and inequities in such factors may result in social exclusion, inequalities and non-climatic challenges to health and wellbeing, economic development and basic human rights (Adger et al., 2014; Olsson et al., 2014; Smith et al., 2014). Those less advantaged often also have reduced access to and control over the social, financial, technological and environmental resources that are required for adaptation and transformation (Oppenheimer et al., 2014; AMAP, 2015), thus limiting options for coping and adapting to change (Hijioka et al., 2014). However, even populations with greater wealth and privilege can be vulnerable to some climate change risks (Cardona et al., 2012; Smith et al., 2014), especially if sources of wealth and wellbeing depend upon established infrastructure that is poorly suited to ocean or cryosphere change.

Institutions and governance can shape vulnerability and adaptive capacity, and it can be challenging for weak governance structures to respond effectively to extreme or persistent climate change hazards (Sections 6.4 and 6.9; Cross-Chapter Box 3 in Chapter 1; Berrang-Ford et al., 2014; Hijioka et al., 2014). Furthermore, populations can be negatively impacted by inappropriate climate change mitigation and/or adaptation policies, particularly ones that further marginalise their knowledge, culture, values and livelihoods (Field et al., 2014; Cross-Chapter Box 4 in Chapter 1).

Vulnerability is not static in place and time, nor homogeneously experienced. The vulnerabilities of individuals, groups, and populations to climate change is dynamic and diverse, and reflects changing societal and environmental conditions (Thomas et al., 2019). SROCC examines vulnerability following the conceptual definition presented in Cross-Chapter Box 2 in Chapter 1, and vulnerability in human systems is treated in relative rather than absolute terms.

\subsection{Addressing the Causes and Consequences of Climate Change for the Ocean and Cryosphere}

Effective and ambitious mitigation of climate change would be required to meet the temperature goal of the Paris Agreement (UNFCCC, 2015; IPCC, 2018). Similarly, effective and ambitious adaptation to climate change impacts on the ocean and cryosphere is necessary to enable CRDPs that minimise residual risk, and loss and damage (very high confidence; Cross-Chapter Box 2 in Chapter 1; IPCC, 2018). Mitigation refers to human actions to limit climate change by reducing the emissions and enhancing the sinks of greenhouse gases. Adaptation refers to processes of adjustment by natural or human systems to actual or expected climate and its effects, intended to moderate harm or exploit beneficial opportunities. The presidency of the 23rd Conference Of the Parties of United Nations Framework Convention on Climate Change (UNFCCC) introduced the oceans pathway into the climate solution space, acknowledging both the importance of the ocean in the climate system and that ocean commitments for adaptation and mitigation are available through Nationally Determined Contributions under the UNFCCC (Gallo et al., 2017).

\subsubsection{Mitigation and Adaptation Options in the Ocean and Cryosphere}

Mitigation and adaptation pathways to avoid dangerous anthropogenic interference with the climate system (United Nations, 


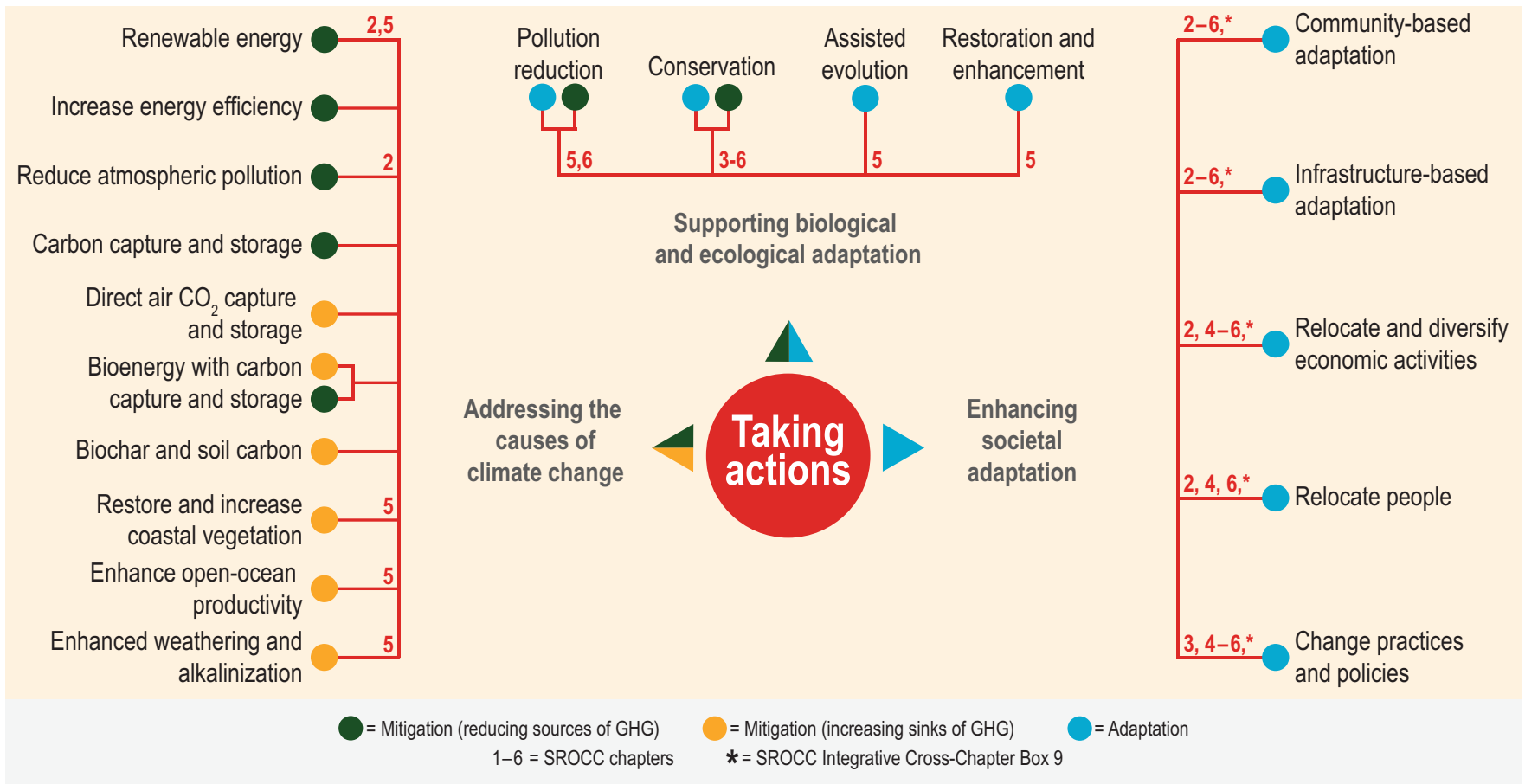

Figure 1.2 Overview of the main ocean-cryosphere mitigation and adaptation measures to observed and expected changes in the context of this report. A longer description of these measures are given in SM1.3. Solar radiation management techniques are omitted because they are covered in other IPCC 6th Assessment Report (AR6) products. Governance and enabling conditions are implicitly embedded in all mitigation and adaptation measures. Some governance-based measures (e.g., institutional arrangements) are not included in this figure but are covered in Cross-Chapter Box 3 in Chapter 1 and in Chapters 2 to 6. GHG: greenhouse gases. Modified from Gattuso et al. (2018).

1992) are considered in SR15 (IPCC, 2018). SROCC assesses several ocean and cryosphere-specific measures for mitigation and adaptation including options for to address the causes of climate change, support biological and ecological adaptation, and enhance societal adaptation (Figure 1.2). Other measures have been proposed, including solar radiation management and several other forms of carbon dioxide removal, but these are not addressed in SROCC as they are covered in other products of the IPCC Sixth Assessment Cycle (SR15 and AR6 Working Group III) and are outside the scope of SROCC. SROCC does assess indirect mitigation measures that involve the ocean and the cryosphere (Figure 1.2) by supporting biological and ecological adaptation, such as through reducing nutrient and organic carbon pollution (which moderates ocean acidification in eutrophied areas) and conservation (which preserves biodiversity and habitats) in coastal regions (Billé et al., 2013).

A literature-based expert assessment shows that ocean-related mitigation measures have trade-offs, with the greatest benefits derived by combining global and local measures (high confidence; Gattuso et al., 2018). Local measures, such as pollution reduction and conservation, provide significant co-benefits and few adverse side effects (high confidence; Sections 5.5.1, 5.5.2). They can be relatively rapidly implemented, but are generally less effective in addressing the global problem (high confidence; Sections 5.5.1, 5.5.2). Likewise, local efforts to decrease air pollution near mountain glaciers and other cryosphere components, for example reducing black carbon emissions, can bring regional-scale benefits for health and in reducing snow and ice-melt (Shindell et al., 2012; Box 2.2).
Well-chosen human interventions can enhance the adaptive capacity of natural systems to climate change. Such interventions through manipulating an ecosystem's structural or functional properties (e.g., restoration of mangroves) may minimise climate change pressures, enhance natural resilience and/or re-direct ecosystem responses to reduce cascading risks on societies. In human systems, adaptation can involve both infrastructure (e.g., enhanced sea defences) and community-based action (e.g., changes in policies and practices). Adaptation options to ongoing climate change are most effective when considered together with mitigation strategies because there are limits to effective adaptation, mitigation actions can make adaptation more difficult, and some adaptation measures may increase greenhouse gas emissions.

Adaptation and mitigation decisions are connected with economic concerns. In SROCC, two main economic approaches are used. The first comprises the Total Economic Value method and the valuation of ecosystem services. SROCC considers the paradigm of sustainable development, and the linkages between climate impacts on ecosystem services (Section 5.4.1) and the consequences on SDGs including food security or poverty eradication (Section 5.4.2). The second economic approach used are formal decision analysis methods, which help to identify options (also called alternatives) that perform best or well with regards to given objectives. These methods include cost-benefit analysis, multi-criteria analysis and robust decision-making and are specifically relevant for appraising long-term investment decisions in the context of coastal adaptation (Section 4.4.4.6). 


\subsubsection{Adaptation in Natural Systems, Ecosystems, and Human Systems}

In AR5, a range of changes in ocean and cryosphere natural systems were linked with medium to high confidence to pressures associated with climate change (Cramer et al., 2014). Climate change impacts on natural ecosystems are variable in space and time. The multiplicity of pressures these natural systems experience impedes attribution of population or ecosystem responses to a specific ocean and/or cryosphere change. Moreover, the interconnectivity of populations within ecosystems means that a single 'adaptive response' of a population, or the aggregate response of an ecosystem (the adaptive responses of the interconnected populations), is influenced not just by direct pressures of climate change, but occurs in concert with the adaptive responses of other species in the ecosystem, further complicating efforts to disentangle specific patterns of adaptation.

Notwithstanding the network of pressures and adaptations, much effort has gone into resolving the mechanisms, interactions and feedbacks of natural systems associated with the ocean and cryosphere. Chapters 4, 5 and 6, as well as Cross-Chapter Box 9, assess new knowledge on the adaptive responses of wetlands, coral reefs, other coastal habitats, and the populations of marine organisms encountering ocean-based risks, including. Likewise, Chapters 2 and 3 describe emerging knowledge on how ecosystems in high-mountain and polar areas are adapting to cryosphere decline.

AR5 and SR15 have highlighted the importance of evolutionary adaptation as a component of how populations adapt to climate change pressures (e.g., Pörtner et al., 2014; Hoegh-Guldberg et al., 2018). Acclimatisation (variation in morphology, physiology or behaviour) can result from changes in gene expression but does not involve change in the underlying DNA sequence. Responses related to acclimatisation can occur both within single generations and over several generations. In contrast, evolution requires changes in the genetic composition of a population over multiple generations; for example, by differential survival or fecundity of different genotypes (Sunday et al., 2014). Adaptive evolution is the subset of evolution attributable to natural selection, and natural selection may lead to populations becoming more fit (Sunday et al., 2014) or extend the range of environments where populations persist (van Oppen et al., 2015). The efficacy of natural selection is affected by population size (Charlesworth, 2009), standing genetic variation, the ability of a population to generate novel genetic variation, migration rates and the frequency of genetic recombination (Rice, 2002). Many studies have shown evolution of traits within and across life stages of populations (Pespeni et al., 2013; Hinners et al., 2017), but there are fewer studies on how evolutionary change can impact ecosystem or community function, and whether trait evolution is stable (Schaum and Collins, 2014). Although acclimatisation and evolutionary adaptation are separate processes, they influence each other, and both adaptive and maladaptive variation of traits can facilitate evolution (Schaum and Collins, 2014; Ghalambor et al., 2015). Natural evolutionary adaptation may be challenged by the speed and magnitude of current ocean and cryosphere changes, but emerging studies investigate how human actions may assist evolutionary adaptation and thereby possibly enhance the resilience of natural systems to climate change pressures (e.g., Box 5.4 in Section 5.5.2). Through acclimatisation and evolutionary adaptation to the pressures from climate change (and all other persistent pressures), populations, species and ecosystems present a constantly changing context for the adaptation of human systems to climate change.

There are several human adaptation options for climate change impacts on the ocean and cryosphere. Adaptive responses include nature-and ecosystem-based approaches (Renaud etal., 2016; Serpetti et al., 2017). Additionally, more social-based approaches for human adaptation range from community-based and infrastructure-based approaches to managed retreat, along with other forms of internal migration (Black et al., 2011; Hino et al., 2017). Building on AR5 (Wong et al., 2014), Chapter 4 describes four main modes of adaptation to mean and extreme sea level rise: protect, advance, accommodate, and retreat. This report demonstrates that all modes of adaptation include mixes of institutional, individual, socio-cultural, engineering, behavioural and/or ecosystem-based measures (e.g., Section 4.4.2).

The effectiveness and performance of different adaptation options across spatial and social scales is influenced by their social acceptance, political feasibility, cost-efficiency, co-benefits and trade-offs (Jones et al., 2012; Adger et al., 2013; Eriksen et al., 2015). Scientific evaluation of past successes and future options, including understanding barriers, limits, risks and opportunities, are complex and inadequately researched (Magnan and Ribera, 2016). In the end, adaptation priorities will depend on multiple parameters including the extent and rate of climate change, the risk attitudes and social preferences of individuals and institutions (and the returns they may gain) (Adger et al., 2009; Brügger et al., 2015; Evans et al., 2016; Neef et al., 2018) and access to finances, technology, capacity and other resources (Berrang-Ford et al., 2014; Eisenack et al., 2014).

Since AR5, transformational adaptation (i.e., the need for fundamental changes in private and public institutions and flexible decision-making processes to face climate change consequences) has been increasingly studied (Cross-Chapter Box 2 in Chapter 1). The recent literature documents how societies, institutions, and/or individuals increasingly assume a readiness to engage in transformative change, via their acceptance and promotion of fundamental alterations in natural or human systems (Klinsky et al., 2016). People living in and near coastal, mountain and polar environments often pioneer these types of transformations, since they are at the forefront of ocean and cryosphere change (e.g., Solecki et al., 2017). Community led and indigenous led adaptation research continues to burgeon (Ayers and Forsyth, 2009; David-Chavez and Gavin, 2018), especially in many mountain (Section 2.3.2.3), Arctic (Section 3.5), and coastal (Section 4.4.4.4, 4.4.5.4, Cross-Chapter Box 9) areas, and demonstrate potential for enabling transformational adaptation (Dodman and Mitlin, 2013; Chung Tiam Fook, 2017). Similarly, the concepts of scenario planning and 'adaptation pathway' design have expanded since AR5, especially in the context of development planning for coastal and delta regions (Section 4.4, Cross-Chapter Box 9; Wise et al., 2014; Maier et al., 2016; Bloemen et al., 2018; Flynn et al., 2018; Frame et al., 2018; Lawrence et al., 2018). 


\subsection{Governance and Institutions}

SROCC conceptualises governance as deciding, managing, implementing and monitoring policies in the context of ocean and cryosphere change. Institutions are defined as formal and informal social rules that shape human behaviour (Roggero et al., 2017). Governance guides how different actors negotiate, mediate their interests and share their rights and responsibilities (Forino et al., 2015; See SROCC Annex I: Glossary and Cross-Chapter Box 3 in Chapter 1 for definition). Governance and institutions interface with climate and social-ecological change process across local, regional and global scales (Fischer et al., 2015; Pahl-Wostl, 2019).

SROCC explores how the interlinked social-ecological systems affect challenge current governance systems in the context of ocean and cryosphere change. These challenges include three aspects. First, the scale of changes to ocean and cryosphere properties driven by global warming, and in the ecosystems they support and services they provide, are poorly matched to existing scales of governance (Sections 2.2.2.1; 2.3.1.3; 3.2.1; 3.5.3). Second, the nature of changes in ecosystem services resulting from changes in ocean and cryosphere properties, including services provided to humans living far from the mountains and coasts, are poorly matched to existing institutions and processes of governance (Section 4.4.4). Third, many possible governance responses to these challenges could be of limited or diminished effectiveness unless they are coordinated on scales beyond that of currently available governance options (Section 6.9.2; Box 5.5).

Hydrological processes in the high mountain cryosphere connect through upstream and downstream areas of river basins (Molden et al., 2016; Chen et al., 2018), including floodplains and deltaic regions (Kilroy, 2015; Cross-Chapter Box 3 in Chapter 1). These cross boundary linkages challenge local-scale governance and institutions that determine how the river-based ecosystem services that sustain food, water and energy are used and distributed (Rasul, 2014; Warner, 2016; Lele et al., 2018; Pahl-Wostl et al., 2018). Small Island States face rising seas that threaten habitability of their homeland and the possibility of losing their nation-state, cultural identity and voices in international governance (Gerrard and Wannier, 2013; Philip, 2018; Section 1.4, Cross-Chapter Box 9), highlighting the need for transboundary components to governance.

These governance challenges cannot be met without working across multiple organisations and institutions, bringing varying capacities, frameworks and spatial extents (Cross-Chapter Box 3 in Chapter 1). Progress in governance for ocean and cryosphere change will require filling gaps in legal frameworks (Amsler, 2016), aligning spatial mismatches (Eriksen et al., 2015; Young, 2016; Cosens et al., 2018), improving the ability for nations to cooperate effectively (Downie and Williams, 2018; Hall and Persson, 2018) and integrating across divided policy domains, most notably of climate change adaptation and disaster risk reduction (e.g., where slow sea level change also alters the implications for civil defense planning and the management of extreme events; Mysiak et al., 2018).

Harmonising local, regional and global governance structures would provide an overarching policy framework for action and allocation of necessary resources for adaptation. Coordinating the top-down and bottom-up governance processes (Bisaro and Hinkel, 2016; Sabel and Victor, 2017; Homsy et al., 2019) to increase effectiveness of responses, mobilise and equitably distribute adequate resources and access private and public sector capabilities requires a polycentric approach to governance (Ostrom, 2010; Jordan et al., 2015). Polycentric governance connotes a complex form of governance with multiple centres of decision making working with some degree of autonomy (Carlisle and Gruby, 2017; Baldwin et al., 2018; Mewhirter et al., 2018; Hamilton and Lubell, 2019).

\section{Cross-Chapter Box 3 | Governance of the Ocean, Coasts and the Cryosphere under Climate Change}

Authors: Anjal Prakash (Nepal/India), Sandra Cassotta (Denmark), Bruce Glavovic (New Zealand/South Africa), Jochen Hinkel (Germany), Elisabeth Holland (Fij/USA), Md Saiful Karim (Australia/Bangladesh), Ben Orlove (USA), Beate Ratter (Germany), Jake Rice (Canada), Evelia Rivera-Arriaga (Mexico), Catherine Sutherland (South Africa)

This Cross-Chapter Box outlines governance and associated institutional challenges and emerging solutions relevant to the ocean, coasts and cryosphere in a changing climate. It illustrates these through three cases: (Case 1) multi-level interactions in Ocean and Arctic governance; (Case 2) mountain governance; and (Case 3) coastal risk governance. Governance refers to how political, social, economic and environmental systems and their interactions are governed or 'steered' by establishing and modifying institutional and organisational arrangements which regulate social processes, mitigate conflicts and realise mutual gains (North, 1990; Pierre and Peters, 2000; Paavola, 2007). Institutions are formal and informal rules and norms, constructed and held in common by social actors that guide, constrain and shape human interactions (North, 1990; Ostrom, 2005). Formal institutions include constitutions, laws, policies and contracts, while informal institutions include customs, social norms and taboos. Both administrative or state government structures and indigenous or traditional governance structures govern the ocean, coasts and cryosphere.

\section{Understanding governance in a changing climate}

The Special Report on the Ocean and Cryosphere in a Changing Climate (SROCC), together with the Special Report on Global Warming of $1.5^{\circ} \mathrm{C}$ (SR15) (IPCC, 2018), highlights the critical role of governance in implementing effective climate adaptation. Chapter 2 explores local community institutions offering autonomous adaptation in the Alps, Andes, Himalayas and other mountain 
Cross-Chapter Box 3 (continued)

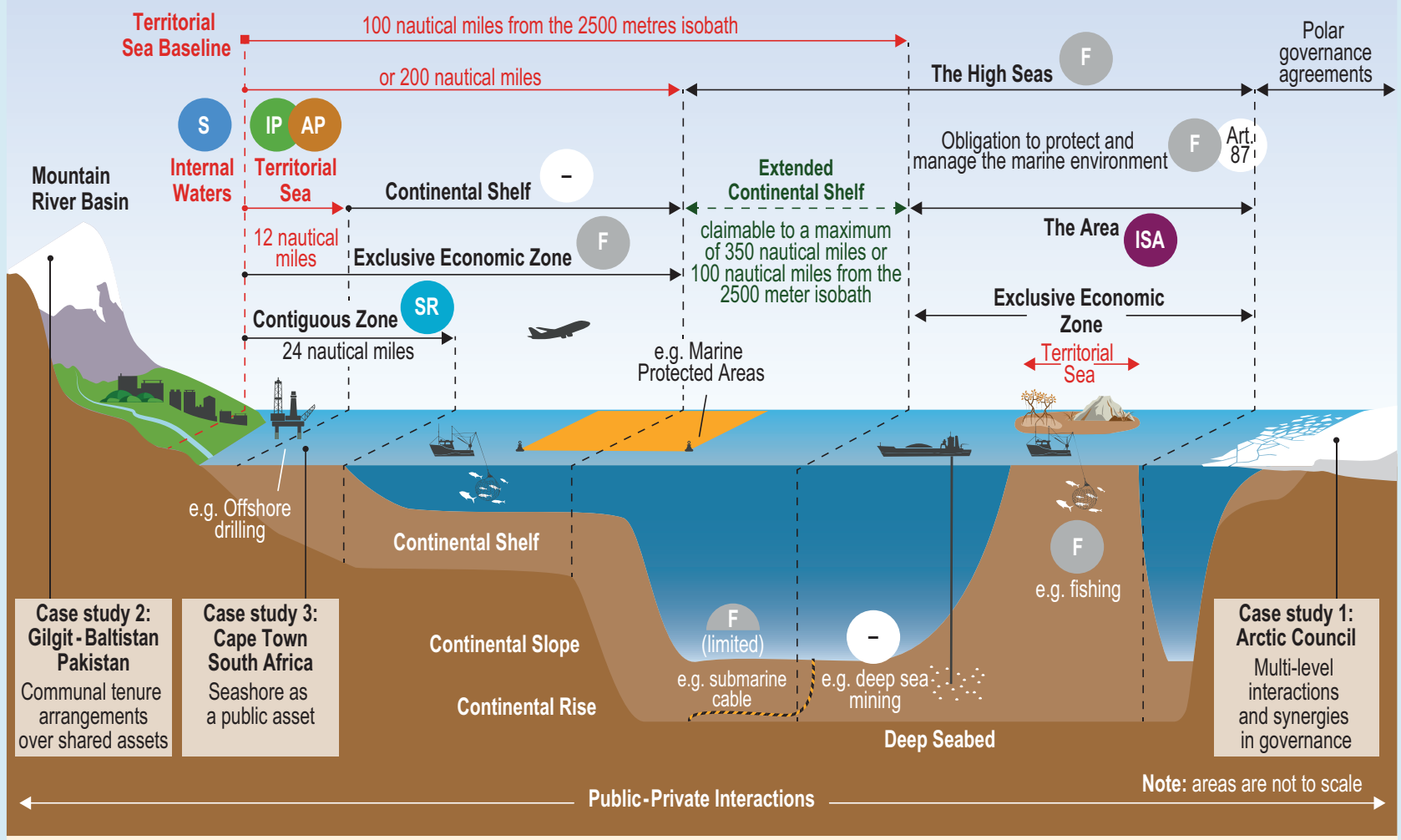

\section{Sovereignty sovereign rights and freedoms in marine zones}

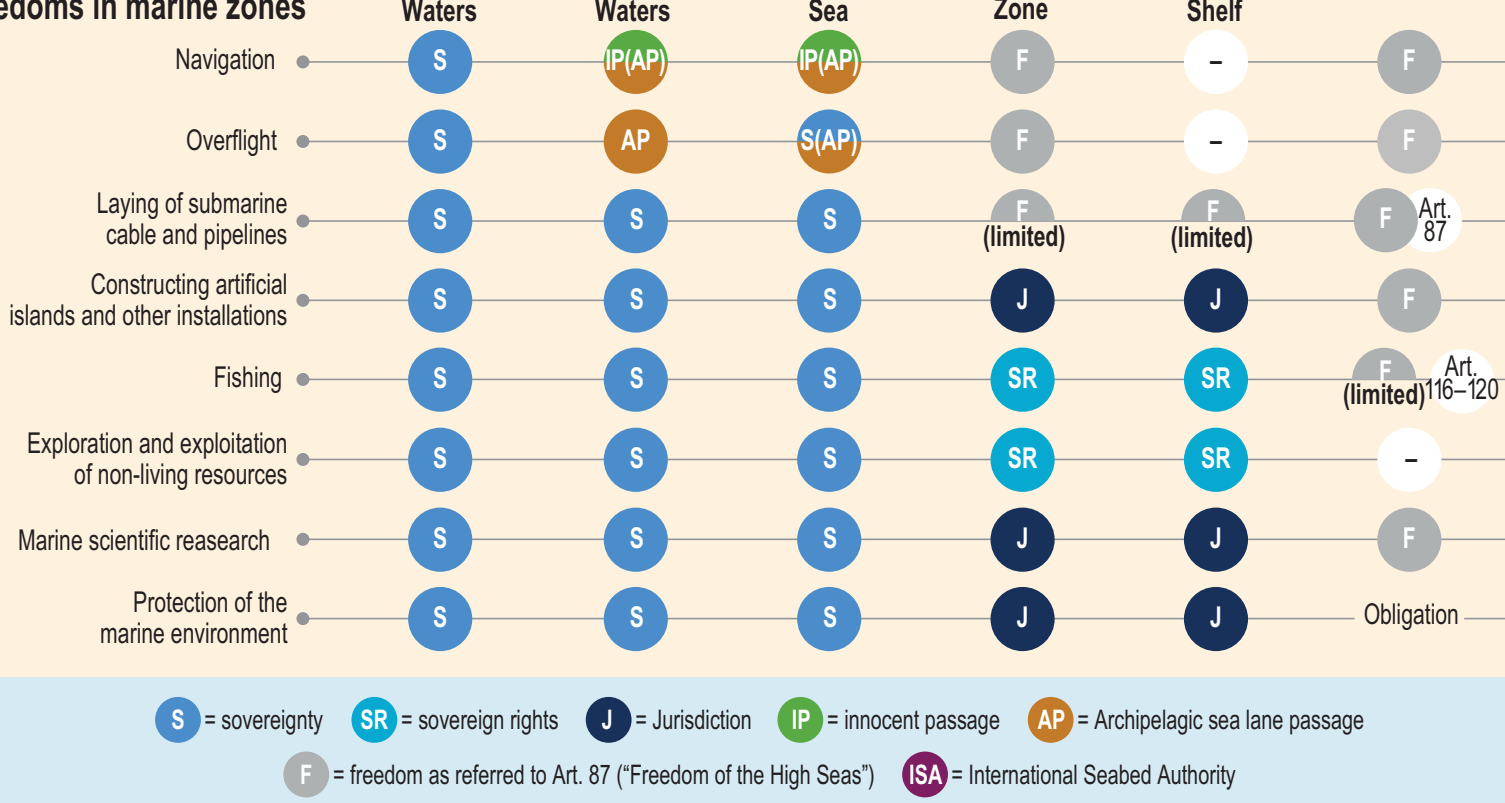

Figure CB3.1 | Spatial distribution of multi-faceted governance arrangements for the ocean, coasts and cryosphere (Panel A) sovereignty, sovereign rights, jurisdictions and freedoms defined for different ocean zones and sea by the United Nations Convention on the Law of the Sea (UNCLOS) (Panel B). Figure CB3.1 is designed to be illustrative and is not comprehensive of all governance arrangements for the ocean, coasts and cryosphere. 
regions (Section 2.4), focusing on the need for transboundary cooperation to support water governance and mitigate conflict. Chapter 3 explores how polar governance system facilitate building resilient pathways, knowledge co-production, social learning, adaptation and power-sharing with Indigenous Peoples at the regional level. This would help in increasing international cooperation in multi-level governance arenas to strengthen responses supporting adaptation in socio-ecological systems (Section 3.5.4). Chapter 4 illustrates how sea level rise governance attempts to address conflicting interests in coastal development, risk management and adaptation with a diversity of governance contexts and degrees of community participation, with a focus on equity concerns and inevitable trade-offs (Section 4.4). Chapter 5 includes a review of existing international legal regimes for addressing ocean warming, acidification and deoxygenation impacts on social-ecological systems and considers ways to facilitate appropriate responses to ocean change (Sections 5.4, 5.5). Chapter 6 explores the issues of credibility, trust, and reliability in government that arise from promoting 'paying the costs of preparedness and prevention' as an alternative to 'bearing the costs of loss and damage' (Section 6.9).

Climate change challenges existing governance arrangements in a variety of ways. First, there are complex interconnections between climate change and other processes that influence the ocean, coasts and cryosphere, making it difficult to untangle climate governance from other governance efforts. Second, the time frames for societal decision-making and government terms are mismatched with the long-term commitment of climate change. Third, governance choices have to be made in the face of uncertainty about the rate and scale of change that will occur in the medium to long-term (Cross-Chapter Box 5 in Chapter 1). Lastly, climate change progressively alters the environment and hence requires continual innovation and adjustment of governance arrangements (Bisaro and Hinkel, 2016; Roggero et al., 2018). Novel transboundary interactions and conflicts are emerging as well as new multi-level governance structures for international and regional cooperation, strengthening shared decision-making among States and other actors (Case 1). The prospects of 'disappearing states', glacier retreat and increasing water scarcity are resulting in States redefining complex water-sharing agreements (Case 2). Coastal risk is escalating, which may require participatory governance responses and the co-production of knowledge at the local scale (Case 3; see also Cross-Chapter Box 9).

Governance, exercised through legal, administrative and other social processes, is essential to prevent, mitigate and adapt to the challenges and risks posed by a changing climate. These governance processes determine roles in the exercising of power and hence decision-making (Graham et al., 2003). Governance may be an act of governments (e.g., passing laws or providing

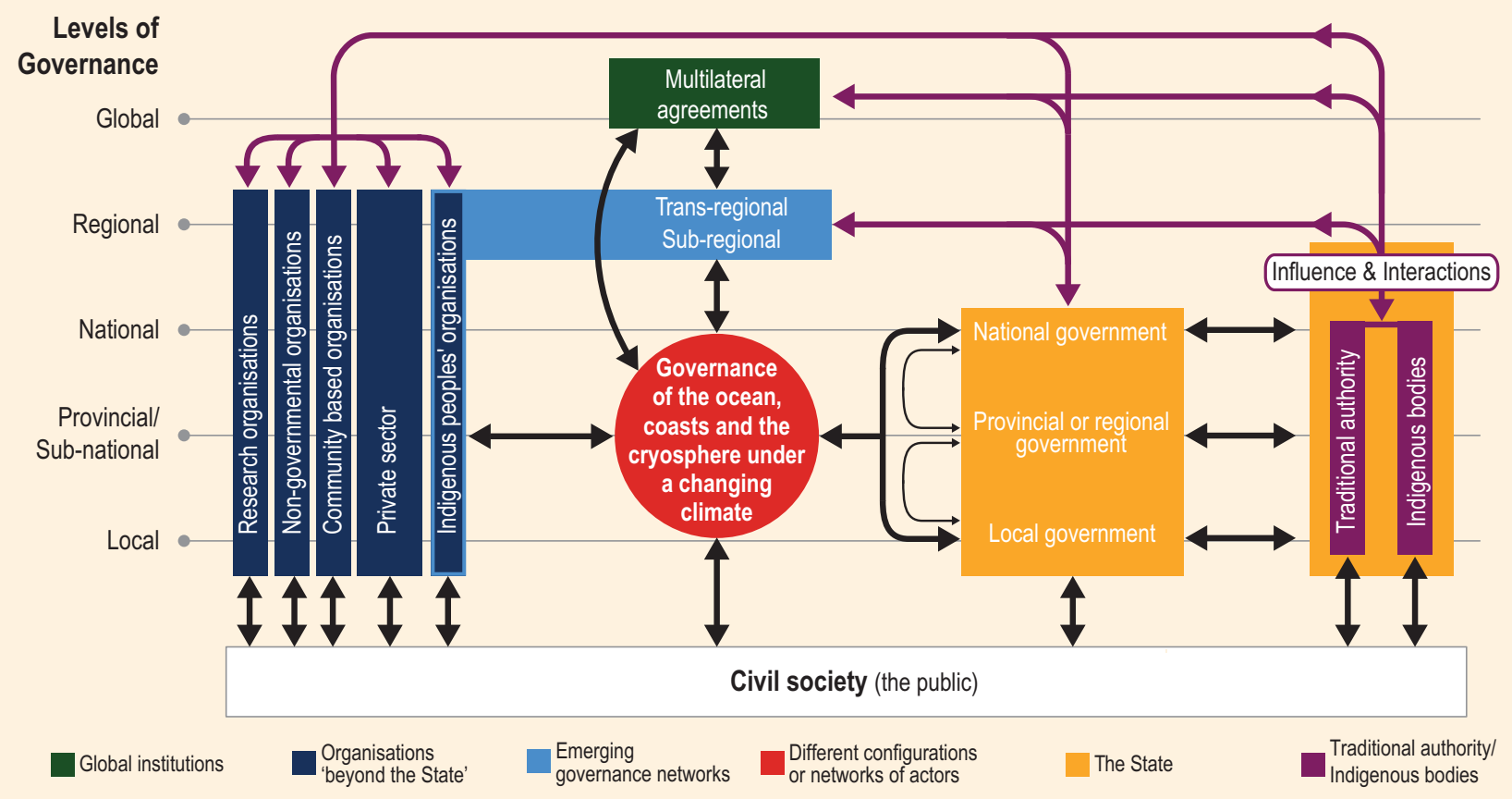

Figure CB3.2 I Interactions and emergence of network governance arrangements for the ocean, coasts and cryosphere across different scales. Adapted from Sommerkorn and Nilsson (2015). 
incentives or information such that citizens can respond more effectively to climate change); private sector actions (e.g., insurance); a co-operative effort among local actors governing themselves through customary law (e.g., by establishing entitlements or norms regulating the common use of scarce resources); a collaborative multi-level effort involving multiple actors (state, private and civil society; e.g., UNFCCC); or a multi-national effort (e.g., Antarctic Treaty; see Figure CB3.2). The complexities of governance arrangements in the ocean, coasts and cryosphere (Figure CB3.1), and the interactions and emergence of relationships between different governance actors in multiple configurations across various spatial scales (Figure CB3.2) are illustrated below.

Case Study 1 - Multi-level Interactions and Synergies in Governance. The UN Convention on the Law of the Sea (UNCLOS) and the changing Arctic: Climate-change induced sea level rise (Section 4.2), could shift the boundaries and territory of some coastal states, changing the areas where their coastal rights are applied under UNCLOS. In extreme cases, inundation from sea level rise might lead to loss of territory and sovereignty, the disappearance of islands and the loss of international maritime jurisdiction subject to maritime claim. These challenges have limited opportunities for recourse in international law and it remains unclear what adequate responses from an international law perspective would be (Vidas et al., 2015; Andreone, 2017; Mayer and Crépeau, 2017; Chircop et al., 2018). While specific legal arrangements and instruments of environmental protection are in place at a regional, sub-regional and national level, they are insufficient to address the new challenges sea level rise brings. Institutional responses to the geopolitical transformation caused by climate change, such as through the Arctic Council (AC) and the 'Law of the Sea' are still evolving. Similar to many international agreements, UNCLOS 'Law of the Sea' provisions for enforcement, compliance, monitoring and dispute settlement mechanisms are not comprehensive, and commonly depend on further, detailed law-making by state parties, acting through competent international organizations (Vidas, 2000; Karim, 2015; De Lucia, 2017; Grip, 2017). Shifts from traditional state-based practices of international law to multi-level and informal governance structures that involve state and non-state actors (including Indigenous Peoples) may address these challenges (medium confidence; Cassotta, 2012; Shadian, 2014; Young, 2016; Andreone, 2017). The AC is a regionally focused governance structure blending new forms of formal and informal multi-level regional cooperation (Young, 2016). The soft law mechanisms employed draw upon best available practice and standards from multiple knowledge systems (Cassotta and Mazza, 2015; Pincus and Ali, 2015) in an attempt to respond to the ocean's global, trans-regional and national climate challenges (Section 3.5.4.2). Reconfiguration and restructuring of the AC has been proposed in order to address emerging trans-regional and global problems (high confidence; Baker and Yeager, 2015; Pincus and Ali, 2015; Young, 2016). Within the existing scope, the AC has amplified the voice of Arctic people affected by the impacts of climate change and mobilized action (Koivurova, 2016). The influence of actors 'beyond the state' is emerging (Figure CB3.2). However, the state retains its importance in tackling the new challenges produced by climate change, as the role of international cooperation in UNCLOS and the Polar Regions demonstrates (Section 3.5.4.2). For example, Article 234 (Ice-covered areas) and Article 197 of the UNCLOS Convention in protecting the marine environment, states that 'States shall cooperate on a global basis and, as appropriate, on a regional basis [...] taking into account characteristic regional features'.

Case Study 2 - Mountain Governance: Water management in Gilgit-Baltistan, Pakistan. Gilgit-Baltistan is an arid territory in a mountainous region of northern Pakistan. Melt water fed streams supply irrigation water for rural livelihoods (Nüsser and Schmidt, 2017). The labour intensive work of constructing and maintaining gravity-fed irrigation canals is done by jirga, traditional community associations. As glaciers retreat due to climate change, water sources at the edge of glaciers have been impacted, reducing water available for irrigation. In response, villagers constructed new channels accessing more distant water for irrigation needs (Parveen et al., 2015). The Aga Khan Development Network supported this substantial task by providing funding and developing a new kind of cross-scale governance network, drawing on local residents for staff (Walter, 2014), and strengthening community resources, training and networks. Challenges remain, including the potential for increased rainfall causing landslides that could damage new canals, and possible expansion of Pakistan's hydropower infrastructure that would further diminish water resources and displace villages (Shaikh et al., 2015). On a geopolitical scale, decreased water supplies from the glaciers could exacerbate tensions over water resources in the region, impacting water management in many parts of the Indus watershed (Uprety and Salman, 2011; Jamir, 2016; see Section 2.3.1.4 for details).

Case Study 3 - Coastal Governance: Risk management for sea level changes in the City of Cape Town, South Africa. Sea level rise and coastal flooding are the focus of the City of Cape Town's coastal climate adaptation efforts. The Milnerton coastline High Water Mark, a non-static line marking the high tide, is creating a governance conflict by moving landwards (due to sea level rise) and intersecting with private property boundaries, threatening public beaches and the dune cordon and placing private property and municipal infrastructure at risk in storm conditions (Sowman et al., 2016). Private property owners are using a mixture of formal, ad hoc, and in some cases illegal, coastal barrier measures to protect their assets from sea level and storm risks, but these are creating additional erosion impacts on the coastline. Legally, the City of Cape Town is not responsible for remediating 
Cross-Chapter Box 3 (continued)

private land impacted by coastal erosion (Smith et al., 2016). However, city officials feel compelled to take action for the common good using a progressive, multi-stakeholder participatory approach. This involves opening up opportunities for dialogue and co-producing knowledge, instead of a purely legalistic and state-centric compliance approach (Colenbrander et al., 2015). The city's actions are both mindful of international frameworks on climate change and responsive to national and provincial legislation and policy. A major challenge that remains is how to navigate the power struggles that will be triggered by this consultative process, as different actors define and negotiate their interests, roles and responsibilities (see Section 4.4.3; Table 4.9).

\section{Conclusions}

These cases illustrate four important points. First, new governance challenges are emerging due to climate change, including: disruptions to long-established cultures, livelihoods and even territorial sovereignty (Case 1); changes in the accessibility and availability of vital resources (Case 2); and the blurring of public and private boundaries of risk and responsibility through accelerated coastal erosion (Case 3; Figure CB3.1). Second, new governance arrangements are emerging to address these challenges, including participatory and networked structures linking formal and informal networks, and involving state, private sector, indigenous and civil society actors in different configurations (Figure CB3.2). Third, climate governance is a complex, contested and unfolding process, with governance actors and networks having to learn from experience, to innovate and develop context-relevant arrangements that can be adjusted in the face of ongoing change. Lastly, there is no single climate governance panacea for the ocean, coasts and cryosphere. Empirical evidence on which governance arrangements work well in which context is still limited, but 'good governance' norms indicate the importance of inclusivity, fairness, deliberation, reflexivity, responsiveness, social learning, the co-production of knowledge and respect for ethnic and cultural diversity.

\subsection{Knowledge Systems for Understanding and Responding to Change}

Assessments of how climate change interacts with the planet and people are largely based on scientific knowledge from observations, theories, modelling and synthesis to understand physical and ecological systems (Section 1.8.1), societies (e.g., Cross-Chapter Box 2 in Chapter 1, Section 1.5) and institutions (e.g., Cross-Chapter Box 3 in Chapter 1). However, humans integrate information from multiple sources to observe and interact with their environment, respond to changes, and solve problems. Accordingly, SROCC also recognises the importance of Indigenous knowledge and local knowledge in understanding and responding to changes in the ocean and cryosphere (Sections 1.8.2, 1.8.3; Cross-Chapter Box 4 in Chapter 1).

\subsubsection{Scientific Knowledge}

\subsubsection{Ocean and Cryosphere Observations}

Long-term sustained observations are critical for detecting and understanding the processes of ocean and cryosphere change (Rhein et al., 2013; Vaughan et al., 2013). Scientific knowledge of the ocean and cryosphere has increased through time and geographical space (Figure 1.3). In situ ocean subsurface temperature and salinity observations have increased in spatial and temporal coverage since the middle of the 19th century (Abraham et al., 2013), and near global coverage $\left(60^{\circ} \mathrm{S}-60^{\circ} \mathrm{N}\right)$ of the upper $2,000 \mathrm{~m}$ has been achieved since 2007 due to the international Argo network (Riser et al., 2016; Figure 1.3). Improved data quality and data analysis techniques have reduced uncertainties in global ocean heat uptake estimates (Sections 1.4.1, 5.2.2). In addition to providing deep ocean measurements, repeated hydrographic physical and biogeochemical observations since AR5 have led to improved estimates of ocean carbon uptake and ocean deoxygenation (Sections 1.4.1, 5.2.2.3, 5.2.2.4). Targeted observational programmes have improved scientific knowledge for specific regions and physical processes of particular concern in a warming climate, including the Greenland and West Antarctic ice sheets (Section 3.3), and the AMOC (Section 6.7). Ocean and cryosphere mass changes and sea level studies have benefited from sustained or newly implemented satellite-based remote sensing technologies, complemented by in situ data such as tide gauges measurements (Sections 3.3, 4.2; Dowell et al., 2013; Raup et al., 2015; PSMSL, 2016). Glacier length measurements in some locations go back many centuries (Figure 1.3), but it is the systematic high resolution satellite monitoring of a large number of the world's glaciers since the late 1970s that has improved global assessments of glacier mass loss (Sections 2.2.3, 3.3.2).

Limitations in knowledge of ocean and cryosphere change remain, creating knowledge gaps for the SROCC assessment. Ocean and cryosphere datasets are frequently short, and do not always span the key IPCC assessment time intervals (Cross-Chapter Box 1 in Chapter 1), so for many parameters the full magnitude of changes since the pre-industrial period is not observed (Figure 1.3). The brevity of ocean and cryosphere measurements also means that some expected changes cannot yet be detected with confidence in direct observations (e.g., Antarctic sea ice loss in Section 3.2.1, AMOC weakening in Section 6.7.1), or other observed changes cannot yet be robustly attributed to anthropogenic factors (e.g., ice sheet mass loss in Section 3.3.1). Observations for many key ocean variables (Bojinski et al., 2014), such as ocean currents, surface heat fluxes, oxygen, inorganic carbon, subsurface salinity, phytoplankton biomass and diversity, etc., do not yet have global coverage or have not reached 
the required density or accuracy for detection of change. Some ocean and cryosphere areas remain difficult to observe systematically, for example, the ocean under sea ice, subsurface permafrost, high mountain areas, marginal seas, coastal areas (Section 4.2.2.3) and ocean boundary currents (Hu and Sprintall, 2016), basin interconnections (Section 6.6) and the Southern Ocean (Sections $3.2,5.2 .2$ ). Measurements that reflect ecosystem change are often location or species specific, and assessments of long-term ocean ecosystem changes are currently only feasible for a limited subset of variables, for example coral reef health (e.g., coral reef health) (Section 5.3; Miloslavich et al., 2018). The deep ocean below 2,000 m is still rarely observed (Talley et al., 2016), limiting (for example) the accurate estimate of deep ocean heat uptake and, consequently the full magnitude of Earth's energy imbalance (e.g., von Schuckmann et al., 2016; Johnson et al., 2018; Sections 1.2, 1.4, 5.2.2).

\subsubsection{Reanalysis Products}

Advances have been made over the past decade in developing more reliable and more highly resolved ocean and atmosphere reanalysis products. Reanalysis products combine observational data with numerical models through data assimilation to produce physically consistent, and spatially complete ocean and climate products (Balmaseda et al., 2015; Lellouche et al., 2018; Storto et al., 2018; Zuo et al., 2018). Ocean reanalyses are widely used to understand changes in physical properties (Section 3.2.1, 5.2), extremes (Sections 6.3 to 6.6), circulation (Section 6.6, 6.7) and to provide climate diagnostics (Wunsch et al., 2009; Balmaseda et al., 2013; Hu and Sprintall, 2016; Carton et al., 2018). Reanalysis products are used in SROCC for assessing climate change process that cause changes in the ocean and cryosphere (e.g., Sections 2.2.1, 3.2.1, 3.3.1, 3.4.1, 5.2.2, 6.3.1, 6.6.1, 6.7.1). Improvements in reanalysis products provide more realistic forcing for regional models, which are used for assessing regional ocean and cryosphere changes that cannot be resolved in global-scale models (e.g., Section 2.2.1; Mazloff et al., 2010; Fenty et al., 2017). The weather forecasts, and seasonal to decadal predictions building on reanalysis products have important applications in the early warning systems that reduce risk and aid human adaptation to extreme events (Sections 6.3.4, 6.4.3, 6.5.3, $6.7 .3,6.8 .5)$.

\subsubsection{Model Simulation Data}

Models are numerical approximations of the Earth system that allow hypotheses about the mechanisms of ocean and cryosphere change to be tested, support attribution of observed changes to specific forcings (Section 1.3), and are the best available information for assessing future change (Figure 1.3). General Circulation Models (GCMs) typically simulate the atmosphere, ocean, sea ice, and land surface, and sometimes also incorporate terrestrial and marine ecosystems. Earth System Models (ESM) are climate models that explicitly include the carbon cycle and may include additional components (e.g., atmospheric chemistry, ice sheets, dynamic vegetation, nitrogen cycle, but also urban or crop models). The systematic set of global-scale model experiments (Taylor et al., 2012) used in SROCC were produced by CMIP5 (Cross-Chapter Box 1 in Chapter 1), including both GCMs and ESMs.
Models may differ in their spatial resolution, and in the extent to which processes are explicitly represented or approximated (parameterised). Model output can be biased due to uncertainties in their physical equations or parameterisations, specification of initial conditions, knowledge of external forcing factors, and unaccounted processes and feedbacks (Hawkins and Sutton, 2009; Deser et al., 2012; Gupta et al., 2013; Lin et al., 2016). Since AR5 there have been advances in modelling the dynamical processes of the Greenland and Antarctica ice sheets, leading to better representation of the range of potential future sea level rise scenarios (Sections 4.2.3). Downscaling, including the use of regional models, makes it possible to improve the spatial resolution of model output in order to better resolve past and future climate change in specific areas, such as high mountains and coastal seas (e.g., Sections 2.2.2, 3.2.3, 3.5.4, 4.2.2, 6.3.1). For biological processes, such as nutrient levels and organic matter production, model uncertainty at regional scales is the main issue limiting confidence in future projections (Sections 5.3, 5.7). While model projections of range shifts for fishes agree with theory and observations, at a regional scale there are known deficiencies in the ways models represent the impacts of ocean variables such as temperature and productivity (Sections 5.2.3, 5.7).

\subsubsection{Palaeoclimate Data}

Palaeoclimate data provide a way to establish the nature of ocean and cryosphere changes prior to direct measurements (Figure 1.3), including natural variability and early anthropogenic climate change (Masson-Delmotte et al., 2013; Abram et al., 2016). Palaeoclimate records utilise the accumulation of physical, chemical or biological properties within natural archives that are related to climate at the time the archive formed. Commonly used palaeoclimate evidence for ocean and cryosphere change comes from marine and lake sediments, ice layers and bubbles, tree growth rings, past shorelines and shallow reef deposits. In many mountain areas, centuries to millennia of palaeoclimate information is now being lost through widespread melting of glacier ice (Cross-Chapter Box 6 in Chapter 2). Palaeoclimate data are spatially limited (Figure 1.3), but often represent regional to global-scale climate patterns, either individually or as syntheses of networks of data (PAGES2K Consortium, 2017).

Palaeoclimate data provide evidence for multi-metre global sea level rises and shifts in climate zones and ocean ecosystems during past warm climate states where temperatures were similar to those expected later this century (Hansen et al., 2016; Fischer et al., 2018; Section 4.2.2). Palaeoclimate reconstructions give context to recent ocean and cryosphere changes that are unusual in the context of variability over past centuries to millennia, including acceleration in Greenland and Antarctic Peninsula ice-melt (Section 3.3.1), declining Arctic sea ice (Section 3.2.1), and emerging evidence for a slowdown of AMOC (Section 6.7.1). Assessments of climate model performance across a wider-range of climate states than is possible using direct observations alone also draws on palaeoclimate data (Flato et al., 2013), and since AR5 important progress has been made to calibrate modelled ice sheet processes and future sea level rise based on palaeoclimate evidence (Cross-Chapter Box 8 in Chapter 3). 


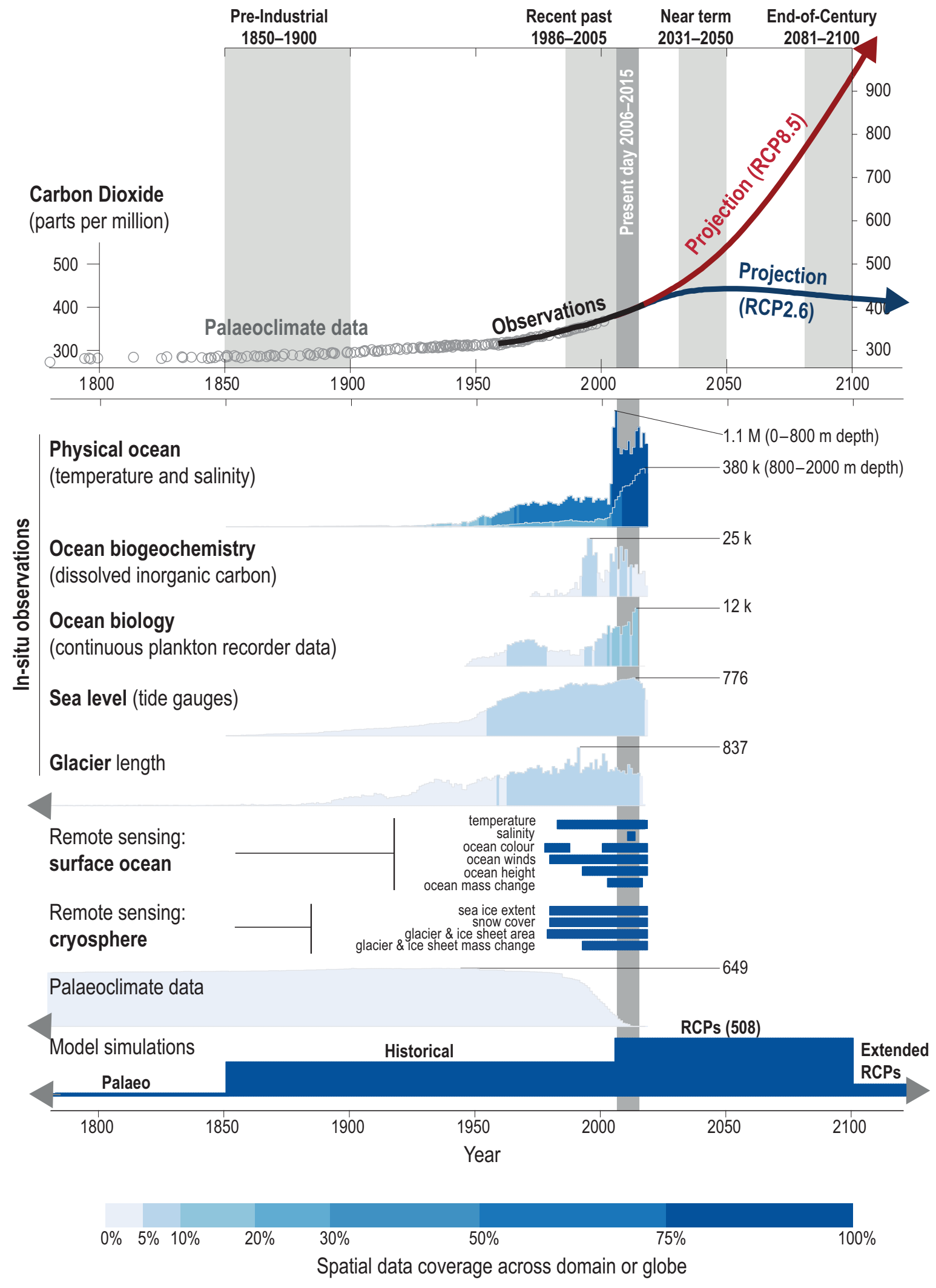

Figure 1.3 | Illustrative examples of the availability of ocean and cryosphere data relative to the major time periods assessed in the Special Report on the Oceans and

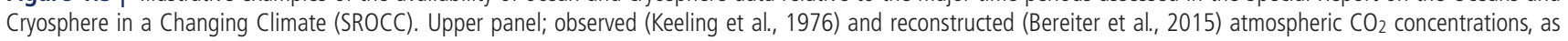
well as the Representative Concentration Pathways (RCPs) of $\mathrm{CO}_{2}$ for low (RCP2.6) and high (RCP8.5) future emission scenarios (van Vuuren et al., 2011a; Cross-Chapter Box 1 in Chapter 1). Lower panel; illustrative examples of data availability for the ocean and cryosphere (Section 1.8.1; Taylor et al., 2012; Boyer et al., 2013; Dowell et al., 2013: McQuatters-Gollop et al., 2015: Raup et al., 2015: Olsen et al., 2016: PSMSL, 2016: PAGES2K Consortium, 2017: WGMS, 2017). The amount of data available through time is shown by the heights of the time series for observational data, palaeoclimate data and model simulations, expressed relative to the maximum annual data availability (maximum values given on plot; $\mathrm{M}=$ million, $\mathrm{k}$ = thousand). Spatial coverage of data across the globe or the relevant domain is shown by colour scale. See SM1.4 for further details. 


\subsubsection{Indigenous Knowledge and Local Knowledge}

Humans create, use, and adapt knowledge systems to interact with their environment (Agrawal, 1995; Escobar, 2001; Sillitoe, 2007), and to observe and respond to change (Huntington, 2000; Gearheard et al., 2013; Maldonado et al., 2016; Yeh, 2016). Indigenous knowledge (IK) refers to the understandings, skills, and philosophies developed by societies with long histories of interaction with their natural surroundings. It is passed on from generation to generation, flexible, and adaptive in changing conditions, and increasingly challenged in the context of contemporary climate change. Local knowledge (LK) is what non-Indigenous communities, both rural and urban, use on a daily and lifelong basis. It is multi-generational, embedded in community practices and cultures and adaptive to changing conditions (FAO, 2018). Each chapter of SROCC cites examples of IK and LK related to ocean and cryosphere change.

IK and LK stand on their own, and also enrich and complement each other and scientific knowledge. For example, Australian Aboriginal groups' Indigenous oral history provides empirical corroboration of the sea level rise 7,000 years ago (Nunn and Reid, 2016), and their seasonal calendars direct hunting, fishing, planting, conservation and detection of unusual changes today (Green et al., 2010). LK works in tandem with scientific knowledge, for example, as coastal Australian communities consider the impacts and trade-offs of sea level rise (O'Neill and Graham, 2016).

Both IK and LK are increasingly used in climate change research and policy efforts to engage affected communities to facilitate site-specific understandings of, and responses to, the local effects of climate change (Hiwasaki et al., 2014; Hou et al., 2017; Mekonnen et al., 2017). IK and LK enrich CRDPs particularly by engaging multiple stakeholders and the diversity of socioeconomic, cultural and linguistic contexts of populations affected by changes in the ocean and cryosphere (Cross-Chapter Box 4 in Chapter 1).

Global environmental assessments increasingly recognise the importance of IK and LK (Thaman et al., 2013; Beck et al., 2014; Díaz et al., 2015). References to IK in IPCC assessment reports increased $60 \%$ from AR4 to AR5, and highlighted the exposures and vulnerabilities of Indigenous populations to climate change risks related to socioeconomic status, resource-based dependence and geographic location (Ford et al., 2016a). All four IPBES assessments in 2018 (IPBES, 2018a; IPBES, 2018b; IPBES, 2018c; IPBES, 2018d) engaged IK and LK (Díaz et al., 2015; Roué and Molnar, 2017; Díaz et al., 2018). Peer-reviewed research on IK and LK is burgeoning (Savo et al., 2016), providing information that can guide responses and inform policy (Huntington, 2011; Nakashima et al., 2012; Lavrillier and Gabyshev, 2018). However, most global assessments still fail to incorporate 'the plurality and heterogeneity of worldviews' (Obermeister, 2017), resulting 'in a partial understanding of core issues that limits the potential for locally and culturally appropriate adaptation responses' (Ford et al., 2016b).

IK and LK provide case specific information that may not be easily extrapolated to the scales of disturbance that humans exert on natural systems (Wohling, 2009). Some forms of IK and LK are also not amenable to being captured in peer-reviewed articles or published reports, and efforts to translate IK and LK into qualitative or quantitative data may mute the multidimensional, dynamic and nuanced features that give IK and LK meaning (DeWalt, 1994; Roncoli et al., 2009; Goldman and Lovell, 2017). Nonetheless, efforts to collaborate with IK and LK knowledge holders (Baptiste et al., 2017; Karki et al., 2017; Lavrillier and Gabyshev, 2017; Roué et al., 2017; David-Chavez and Gavin, 2018) and to systematically assess published IK and LK literature in parallel with scientific knowledge result in increasingly effective usage of the multiple knowledge systems to better characterise and address ocean and cryosphere change (Huntington et al., 2017; Nalau et al., 2018; Ford et al., 2019).

\subsubsection{The Role of Knowledge in People's Responses to Climate, Ocean and Cryosphere Change}

To hold global average temperature to well below $2^{\circ} \mathrm{C}$ above pre-industrial levels, substantial changes in the day-to-day activities of individuals, families, communities, the private sector, and governance bodies will be required (Ostrom, 2010; Creutzig et al., 2018). Enabling these changes at a meaningful societal scale requires sensitivity to communities and their use of multiple knowledge systems to best motivate effective responses to the risks and opportunities posed by climate change (medium confidence) (1.8.2, Cross-Chapter Box 4 in Chapter 1). Meaningful engagement of people and communities with climate change information depends on that information cohering with their perception of how the world works (Crate and Fedorov, 2013). The values and identities people hold affect how acceptable they find the behavioural changes, technological solutions and governance that climate change action requires (Moser, 2016).

Education and climate literacy contribute to climate change action and adaptation (high confidence). Although public understanding of humanity's role in both causing and abating climate change has increased in the last decade (Milfont et al., 2017), levels of climate concern vary greatly globally (Lee et al., 2015). Educational attainment has the strongest effect on raising climate change awareness (Lee et al., 2015), and research documents the value of evidence-based climate change education, particularly during formal schooling (Motta, 2018). People further understand climate change as a serious threat when they experience it in their lives and have knowledge of its human causes (Lee et al., 2015; Shi et al., 2016). Education and tailored climate communication strategies that are respectful of people's values and identity can aid acceptance and implementation of the local to global-scale approaches and policies required for effective climate change mitigation and adaptation (Shi et al., 2016; Anisimov and Orttung, 2018; Sections 3.5.4, 4.4), while also supporting CRDPs (see also Cross-Chapter Box 2 in Chapter 1, and FAQ1.2).

Human psychology complicates engagement with climate change, due to complex social factors, including values (Corner et al., 2014), identity (Unsworth and Fielding, 2014), ideology (Smith and Mayer, 2019) and the framing of climate messaging. Additionally, psychology 
effects adaptation actions, motivated by perceptions that others are already adapting, avoidance of an unpleasant state of mind, feelings of self-efficacy and belief in the efficacy of the adaptation action (van Valkengoed and Steg, 2019). Better understandings of the psychological implications across diverse communities and social and political contexts will facilitate a just transition of both emissions reduction and adaptation (Schlosberg et al., 2017). Impacts of climate change on natural and human environments (e.g., extreme weather) or human-caused modifications to the environment (e.g., adaptation) will raise further psychological challenges. This includes psychological impacts to the emotional wellbeing of people adversely affected by climate change (Ogunbode et al., 2018), resulting in solastalgia (Albrecht et al., 2007), a distress akin to homesickness while in their home environment (McNamara and Westoby, 2011).

\section{Cross-Chapter Box 4 | Indigenous Knowledge and Local Knowledge in Ocean and Cryosphere Change}

Authors: Susan Crate (USA), William Cheung (Canada), Bruce Glavovic (New Zealand), Sherilee Harper (Canada), Hélène Jacot Des Combes (Fiji/France), Monica Ell Kanayuk (Canada), Ben Orlove (USA), Joanna Petrasek MacDonald (Canada), Anjal Prakash (Nepal/India), Jake Rice (Canada), Pasang Yangjee Sherpa (Nepal), Martin Sommerkorn (Norway/Germany)

\section{Introduction}

This Cross-Chapter Box describes how Indigenous knowledge (IK) and local knowledge (LK) are different and unique sources of knowledge, which are critical to observing, responding to, and governing the ocean and cryosphere in a changing climate (See SROCC Annex I: Glossary for definitions). International organisations recognise the importance of IK and LK in global assessments, including UN Environment, UNDP, UNESCO, IPBES, and the World Bank. IK and LK are referenced throughout SROCC, understanding that many climate change impacts affect, and will require responses from, local communities (both Indigenous and non-Indigenous) who maintain a close connection with the ocean and/or cryosphere.

Attention to IK and LK in understanding global change is relatively recent, but important (high confidence). For instance, in 1980, Alaskan Inuit formed the Alaska Eskimo Whaling Commission in response to the International Whaling Commission's science that underestimated the Bowhead whale population and, in 1977, banned whaling as a result (Huntington, 1992). The Commission facilitated an improved population count using a study design based on IK, which indicated a harvestable population (Huntington, 2000). There are various approaches for utilising multiple knowledge systems. For example, the Mi'kmaw Elders' concept of Two Eyed Seeing: which is 'learning to see from one eye with the strengths of Indigenous knowledges, and from the other eye with the strengths of Western [scientific] knowledges, and to use both together, for the benefit of all' (Bartlett et al., 2012), to preserve the distinctiveness of each, while allowing for fuller understandings and actions (Bartlett et al., 2012: 334).

\section{Knowledge Co-production}

Scientific knowledge, IK and LK can complement one another by engaging both quantitative data and qualitative information, including people's observations, responses and values (Huntington, 2000; Crate and Fedorov, 2013; Burnham et al., 2016; Figure CB4.1). However, this process of knowledge co-production is complex (Jasanoff, 2004) and IK and LK possess uncertainties of a different nature from those of scientific knowledge (Kahneman and Egan, 2011), often resulting in the dominance of scientific knowledge over IK and LK in policy, governance and management (Mistry and Berardi, 2016). Working across disciplines (interdisciplinarity; Strang, 2009), and/or engaging multiple stakeholders (transdisciplinarity; Klenk and Meehan, 2015; Crate et al., 2017), are approaches used to bridge knowledge systems. The use of all knowledge relevant to a specific challenge can involve approaches such as: scenario building across stakeholder groups to capture the multiple ways people perceive their environment and act within it (Klenk and Meehan, 2015); knowledge co-production to achieve collaborative management efforts (Armitage et al., 2011); and working with communities to identify shared values and perceptions that enable context-specific adaptation strategies (Grunblatt and Alessa, 2017). Broad stakeholder engagement, including affected communities, Indigenous Peoples, local and regional representatives, policy makers, managers, interest groups and organisations, has the potential to effectively use all relevant knowledge (Obermeister, 2017) and produce results that reduce the disproportionate influence that formally educated and economically advantaged groups often exert in scientific assessments (Castree et al., 2014). 
Cross-Chapter Box 4 (continued)

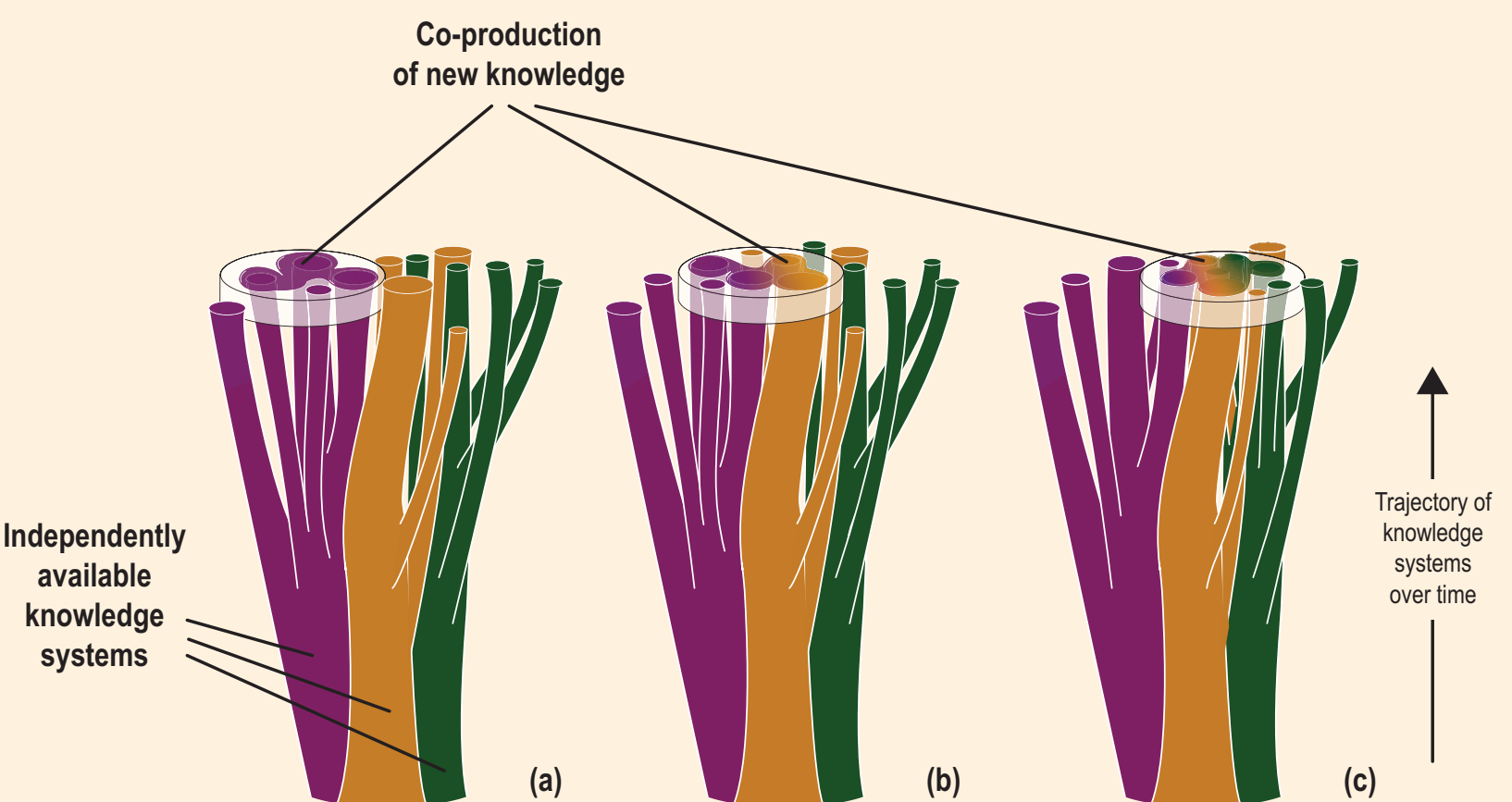

Figure CB4.1 | Knowledge co-production using scientific knowledge, Indigenous knowledge (IK) and/or local knowledge (LK) to create new understandings for decision making. Panels A, B, and C represent the use of one, two, and three knowledge systems, respectively, illustrating co-production moments in time (collars). Panel A represents a context which uses one knowledge system, for example, of IK used by Indigenous peoples; or of LK used by farmers, fishers and rural or urban inhabitants; or of scientific knowledge used in contexts where substantial human presence is lacking. Panel B depicts the use of two knowledge systems, as described in this Cross-Chapter Box in the case of Bowhead whale population counts and in Himalayan flood management. Panel C illustrates the use of all three knowledge systems, as in the Pacific case in this Cross-Chapter Box. Each collar represents how making use of knowledge from different systems is a matter of both identifying available knowledge across systems and of knowledge holder deliberations. In these processes, learning takes place on how to relate knowledge from different systems for the purpose of improved decisions and solutions. Knowledge from different systems can enrich the body of relevant knowledge while continuing independently or can be combined to co-produce new knowledge.

\section{Contributions to SROCC}

Observations, responses, and governance are three important contributions that IK and LK make in ocean and cryosphere change:

Observations: IK and LK observations document glacier and sea ice dynamics, permafrost dynamics, coastal processes, etc. (Sections 2.3.2.2.2. 2.5, 3.2.2, 3.4.1.1, 3.4.1.1, 3.4.1.2, 4.3.2.4.2, 5.2.3 and Box 2.4), and how they interact with social-cultural factors (West and Hovelsrud, 2010). Researchers have begun documenting IK and LK observations only recently (Sections 2.3.1.1, 3.2, 3.4, 3.5, Box 4.4, 5.4.2.2.1).

Responses: Either IK or LK alone (Yager, 2015), or used with scientific knowledge (Nüsser and Schmidt, 2017) inform responses (Sections 2.3.1.3.2, 2.3.2.2.2, 3.5.2, 3.5.4, 4.4.2, Box 4.4, 5.5.2, 6.8.4, 6.9.2). Utilising multiple knowledge systems requires continued development, accumulation, and transmission of IK, LK and scientific knowledge towards understanding the ecological and cultural context of diverse peoples (Crate and Fedorov, 2013; Jones et al., 2016), resulting in the incorporation of relevant priorities and contexts into adaptation responses (Sections 3.5.2, 3.5.4, 4.4.4, 5.5.2, 6.8.4, 6.9.2, Box 2.3).

Governance: Using IK and LK in climate decision and policy making includes customary Indigenous and local institutions (Karlsson and Hovelsrud, 2015), as in the case when Indigenous communities are engaged in an integrated approach for disaster risk reduction in response to cryosphere hazards (Carey et al., 2015). The effective engagement of communities and stakeholders in decisions requires using the multiple knowledge systems available (Chilisa, 2011; Sections 2.3.1.3.2, 2.3.2.3, 3.5.4, 4.4.4, Table 4.4, 5.5.2, 6.8.4, 6.9.2; Sections 2.3.1.3.2, 2.3.2.3, 3.5.4, 4.4.4, Table 4.9, 5.5.2, 6.8.4, 6.9.2). 


\section{Cross-Chapter Box 4 (continued)}

\section{Examples from regions covered in this report}

IK and LK in the Pacific: Historically, Pacific communities, who depend on marine resources for essential protein (Pratchett et al., 2011), use LK for management systems to determine access to, and closure of, fishing grounds, the latter to respect community deaths, sacred sites, and customary feasts. Today a hybrid system, Locally Managed Marine Protected Areas (LMMAs), is common and integrates local governance with NGO or government agency interventions (Jupiter et al., 2014). The expected benefits of these management systems support climate change adaptation through sustainable resource management (Roberts et al., 2017) and mitigation through improved carbon storage (Vierros, 2017). The challenges to wider use include both how to upscale LMMAs (Roberts et al., 2017; Vierros, 2017), and how to assess them as climate change adaptation and mitigation solutions (Rohe et al., 2017; Section 5.4).

IK and Pikialasorsuaq: Pikialasorsuaq (North Water Polynya), in Baffin Bay, is the Arctic's largest polynya, or area of open water surrounded by ice, and is also one of the most biologically productive regions in the Arctic (Barber et al., 2001). Adjacent Inuit communities depend on Pikialasorsuaq for their food security and subsistence economy (Hastrup et al., 2018). They use Qaujimajatuqangit, an IK system, in daily and seasonal activities (ICC, 2017). The sea ice bridge north of the Pikialasorsuaq is no longer forming as reliably as in the past, resulting in a polynya that is geographically and seasonally less defined (Ryan and Münchow, 2017). In response, the Inuit Circumpolar Council initiated the Pikialasorsuaq Commission who formed an Inuit-led management authority to (1) oversee monitoring and research to conserve the polynya's living resources; (2) identify an Indigenous Protected Area, to include the polynya and dependent communities; and (3) establish a free travel zone for Inuit across the Pikialasorsuaq region (ICC, 2017; Box 3.2).

$L K$ in the Alps: Mountain guides and other local residents engaged in supporting mountain tourism draw on LK for livelihood management. A study at Mont Blanc lists specific cryosphere changes which they have observed, including glacial shrinkage and reduction in ice and snow cover. As a result, the categorisation of the difficulty of a number of routes has changed, and the timing of the climbing season has shifted earlier (Mourey and Ravanel, 2017; Section 2.3.5).

$L K$ to manage flooding: Climate change is increasing glacial melt water and rain-induced disasters in the Himalayan region and affected communities in China, Nepal, and India use LK to adapt (Nadeem et al., 2012). For instance, rains upstream in Gandaki (Nepal) flood downstream areas of Bihar, India. Local communities' knowledge of forecasting floods has evolved over time through the complexities of caste, class, gender and ecological flux, and is critical to flood forecasting and disaster risk reduction. Local communities manage risk by using a diverse set of knowledge, including phenomenological (e.g., river sound), ecological (e.g., red ant movement) and riverine (e.g., river colour) indicators, alongside meteorological and official information (Acharya and Prakash, 2018; Section 2.3.2.3).

\section{Knowledge Holders' Recommendations for Utilising IK and LK in Assessment Reports}

Perspectives from the Himalayas: IK and LK holders in the Himalayas have conducted long-term systematic observations in these remote areas for centuries. Contemporary IK details change in phenology, weather patterns, and flora and fauna species, which enriches scientific knowledge of glacial retreat and potential glacial lake outbursts (Sherpa, 2014). The scientific community can close many knowledge gaps by engaging IK and LK holders as counterparts. Suggestions towards this objective are to work with affected communities to elicit their knowledge of change, especially IK and LK holders with more specialised knowledge (farmers, herders, mountain guides, etc.), and use location- and culture-specific approaches to share scientific knowledge and use it with IK and LK.

Perspectives from the Inuit Circumpolar Council (ICC), Canada: Engaging Inuit as partners across all climate research disciplines ensures that Inuit knowledge and priorities guide research, monitoring, and the reporting of results in Inuit homeland. Doing so enhances the effectiveness, impact, and usefulness of global assessments, and ensures that Inuit knowledge is appropriately reported in assessments. Inuit seek to achieve self-determination in all aspects of research carried out in Inuit homeland (e.g., Nickels et al., 2005). Inuit actively produce and use climate research (e.g., ITK, 2005; ICC, 2015) and lead approaches to address climate challenges spurred by great incentive to develop innovative solutions. Engaging Inuit representative organisations and governments as partners in research recognises that the best available knowledge includes IK, enabling more robust climate research that in turn informs climate policy. When interpreted and applied properly, IK comes directly from research by Inuit and from an Inuit perspective (ICC, 2018). This can be achieved by working with Inuit on scoping and methodology for assessments and supporting inclusion of Inuit experts in research, analysis, and results dissemination. 


\subsection{Approaches Taken in this Special Report}

\subsubsection{Methodologies Relevant to this Report}

SROCC assesses literature on ocean and cryosphere change and associated impacts and responses, focusing on advances in knowledge since AR5. The literature used is primarily published, peer-reviewed scientific, social science and humanities research. In some cases, grey literature sources (for example, published reports from governments, industry, research institutes and non-government organisations) are used where there are important gaps in available peer-reviewed literature. It is recognised that published knowledge from many parts of the world most vulnerable to ocean and cryosphere change is still limited (Czerniewicz et al., 2017).

Where possible, SROCC draws upon established methodologies and/or frameworks. Cross-Chapter Boxes in Chapter 1 address methodologies used for projections of future change (Cross-Chapter Box 1 in Chapter 1), for assessing and reducing risk (Cross-Chapter Box 2 in Chapter 1), for governance options relevant to a problem or region (Cross-Chapter Box 3 in Chapter 1), and for using IK and LK (Cross-Chapter Box 4 in Chapter 1). It is recognised in the assessment process that multiple and non-static factors determine human vulnerabilities to climate change impacts, and that ecosystems provide essential services that have both commercial and non-commercial value (Section 1.5). Economic methods are also important in SROCC, for estimating the economic value of natural systems, and for aiding decision-making around mitigation and adaptation strategies (Section 1.6).

\subsubsection{Communication of Confidence in Assessment Findings}

SROCC uses calibrated language for the communication of confidence in the assessment process (Mastrandrea et al., 2010; Mach et al., 2017). Calibrated language is designed to consistently evaluate and communicate uncertainties that arise from incomplete knowledge due to a lack of information, or from disagreement about what is known or even knowable. The IPCC calibrated language uses qualitative expressions of confidence based on the robustness of evidence for a finding, and (where possible) uses quantitative expressions to describe the likelihood of a finding (Figure 1.4).

Qualitative expressions (confidence scale) describe the validity of a finding based on the type, amount, quality and consistency of evidence, and the degree of agreement between different lines of evidence (Figure 1.4, step 2). Evidence includes all knowledge sources, including IK and LK where available. Very high and high confidence findings are those that are supported by multiple lines of robust evidence with high agreement. Low or very low confidence describe findings for which there is limited evidence and/or low agreement among different lines of evidence, and are only presented in SROCC if they address a major topic of concern.

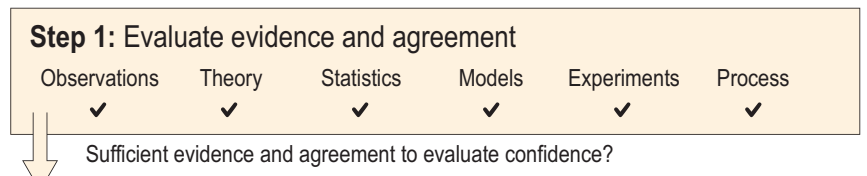

Step 2: Evaluate confidence

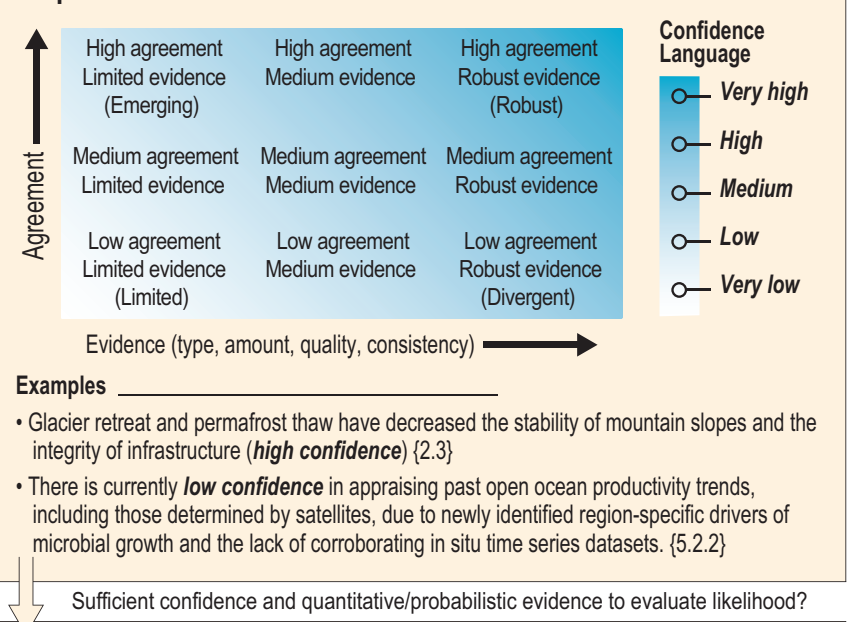

Step 3: Evaluate statistical likelihood

\begin{tabular}{rcc}
$\begin{array}{r}\text { Likelihood } \\
\text { Language }\end{array}$ & $\begin{array}{r}\text { Statistical Level } \\
\text { (assessing change) }\end{array}$ & $\begin{array}{r}\text { Statistical Range } \\
\text { (assessing range) }\end{array}$ \\
\hline $\begin{array}{r}\text { Virtually certain } \\
\text { Extremely likely }\end{array}$ & $>99 \%$ & \\
Very likely & $>95 \%$ & $5-95 \%$ range \\
Likely & $>90 \%$ & $17-83 \%$ range \\
More likely than not & $>66 \%$ & $25-75 \%$ range \\
About as likely as not & $33-66 \%$ & \\
Unlikely & $<33 \%$ & $<17 \%$ and $>83 \%$ (both tails) \\
Very unlikely & $<10 \%$ & $<5 \%$ and $>95 \%$ (both tails) \\
Extremely unlikely & $<5 \%$ & \\
Exceptionally unlikely & $<1 \%$ &
\end{tabular}

Example: statistical level

- Satellite observations reveal that marine heatwaves have very likely doubled in frequency between 1982 and 2016 , and that they have also become longer-lasting, more intense and extensive. $\{6.4\}$

Examples: statistical range

- The AMOC will very likely weaken over the $21 \mathrm{st}$ century (high confidence), although a collapse is very unlikely (medium confidence). Nevertheless, a substantial weakening of the AMOC remains a physically plausible scenario. $\{6.7\}$

- Global mean sea level will rise between $0.43 \mathrm{~m}(0.29-0.59 \mathrm{~m}$, likely range) (RCP2.6) and $0.84 \mathrm{~m}(0.61-1.10 \mathrm{~m}$, likely range (RCP8.5) by 2100 (medium confidence) relative to $1986-2005 .\{4.2 .3\}$

Deep uncertainty (Cross-Chapter Box 5 in Chapter 1)

- Evolution of the Antarctic Ice Sheet beyond the end of the 21 st century is characterised by deep uncertainty as ice sheet models lack realistic representations of some of the underlying physical processes. \{Cross-Chapter Box 8 in Chapter 3 \}

Figure 1.4 | Schematic of the IPCC usage of calibrated language, with examples of confidence and likelihood statements from this report. Figure developed after Mastrandrea et al. (2010), Mach et al. (2017) and Sutton (2018). 
Quantitative expressions (likelihood scale) are used when sufficient data and confidence exists for findings to be assigned a quantitative or probabilistic estimate (Figure 1.4, step 3). In the scientific literature, a finding is often said to be significant if it has a likelihood exceeding $95 \%$ confidence. Using calibrated IPCC language, this level of statistical confidence would be termed extremely likely. Lower levels of likelihood than those derived numerically can be assigned by expert judgement to take into account structural or measurement uncertainties within the products or data used to determine the probabilistic estimates (e.g., Table CB1.1). Likelihood statements may be used to describe how climate changes relate to the ends of distribution functions, such as in detection and attribution studies that assess the likelihood that an observed climate change or event is different to a reference climate state (Section 1.3). In other situations, likelihood statements refer to the central region across a distribution of possibilities. Examples are the estimates of future changes based on large ensembles of climate model simulations, where the central $66 \%$ of estimates across the ensemble (i.e., the $17-83 \%$ range) would be termed a likely range (Figure 1.4, step 3).

It is increasingly recognised that effective risk management requires assessments not just of 'what is most likely' but also of 'how bad things could get' (Mach et al., 2017; Weaver et al., 2017; Xu and
Ramanathan, 2017; Spratt and Dunlop, 2018; Sutton, 2018). In response to the need to reframe policy relevant assessments according to risk (Section 1.5; Mach et al., 2016; Weaver et al., 2017; Sutton, 2018), an effort is made in SROCC to report on potential changes for which there is low scientific confidence or a low likelihood of occurrence, but that would have large impacts if realised (Mach et al., 2017). In some cases where evidence is limited or emerging, phenomena may instead be discussed according to physically plausible scenarios of impact (e.g., Table 6.1).

In some cases, deep uncertainty (Cross-Chapter Box 5 in Chapter 1) may exist in current scientific assessments of the processes, rate, timing, magnitude, and consequences of future ocean and cryosphere changes. This includes physically plausible high-impact changes, such as high-end sea level rise scenarios that would be costly if realised without effective adaptation planning and even then may exceed limits to adaptation. Means such as expert judgement, scenario building, and invoking multiple lines of evidence enable comprehensive risk assessments even in cases of uncertain future ocean and cryosphere changes.

\section{Cross-Chapter Box 5 | Confidence and Deep Uncertainty}

Authors: Carolina Adler (Switzerland/Australia), Michael Oppenheimer (USA), Nerilie Abram (Australia), Kathleen Mclnnes (Australia) and Ted Schuur (USA)

\section{Definition and Context}

Characterising, assessing and managing risks to climate change involves dealing with inherent uncertainties. Uncertainties can lead to complex decision-making situations for managers and policymakers tasked with risk management, particularly where decisions relate to possibilities assessed as having low or unknown confidence/likelihood, yet would have high impacts if realised. While uncertainty can be quantitatively or qualitatively assessed (Section 1.9.2; Figure 1.4), a situation of deep uncertainty exists when experts or stakeholders do not know or cannot agree on: (1) appropriate conceptual models that describe relationships among key driving forces in a system; (2) the probability distributions used to represent uncertainty about key variables and parameters; and/or, (3) how to weigh and value desirable alternative outcomes (adapted from Lempert et al., 2003; Marchau et al., 2019b).

The concept of deep uncertainty has been debated and addressed in the literature for some time, with diverse terminology used. Terms such as great uncertainty (Hansson and Hirsch Hadorn, 2017), contested uncertain knowledge (Douglas and Wildavsky, 1983), ambiguity (Ellsberg, 1961) and Knightian uncertainty (Knight, 1921) among others, are also present in the literature to refer to the multiple components of uncertainty that need to be accounted for in decision making. The purpose of this Cross-Chapter Box is to constructively engage with the concept of deep uncertainty, by first providing some context for how the IPCC has dealt with deep uncertainty in the past. This is followed by examples of cases from the ocean and cryosphere assessments in the Special Report on the Oceans and Cryosphere in a Changing Climate (SROCC), where deep uncertainty has been addressed to advance assessment of risks and their management.

How has the IPCC and other literature dealt with deep uncertainty?

The IPCC assessment process provides instances of how deep uncertainty can manifest. In assessing the scientific evidence for anthropogenic climate change, and its influence on the Earth system in the past and future, IPCC assessments can identify areas where a large range of possibilities exist in the scientific literature or where knowledge of the underlying processes and responses is lacking. Existing guidelines to ensure consistent treatment of uncertainties by IPCC author teams (Mastrandrea et al., 2010; Section 1.9.2) may not be sufficient to ensure the desired consistency or guide robust findings when conditions of deep uncertainty are present (Adler and Hirsch Hadorn, 2014). 
The IPCC, and earlier assessments, encountered deep uncertainty when evaluating numerous aspects of the climate change problem. Examining these cases sheds light on approaches to quantifying and reducing deep uncertainty. An assessment by the US National Academy of Sciences (Charney et al., 1979; commonly referred to as the Charney Report) provides a classic example. Evaluating climate sensitivity to a doubling of carbon dioxide concentration, and developing a probability distribution for it, was challenging because only two 3-D climate models and a handful of model variants and realisations were available. The panel invoked three strategies to eliminate some of these simulations: (1) Using multiple lines of evidence to complement the limited model results; (2) estimating the consequences of poor or absent model representations of certain physical processes (particularly cumulus convection, high-altitude cloud formation, and non-cloud entrainment); and, (3) evaluating mismatches between model results and observations. This triage yielded 'probable bounds' of $2^{\circ} \mathrm{C}-3.5^{\circ} \mathrm{C}$ on climate sensitivity. The panel then invoked expert judgment (Box 12.2 in Collins et al., 2013) to broaden the range to $3 \pm 1.5^{\circ} \mathrm{C}$, with $3^{\circ} \mathrm{C}$ referred to as the 'most probable value'. The panel did not report its confidence in these judgments.

The literature has expanded greatly since, allowing successive IPCC assessments to refine the approach taken in the Charney report. By the IPCC 5th Assessment Report (AR5), four lines of evidence (from instrumental records, palaeoclimate data, model intercomparison of sensitivity, and model-climatology comparisons) were assessed to determine that 'Equilibrium climate sensitivity is likely in the range $1.5^{\circ} \mathrm{C}-4.5^{\circ} \mathrm{C}$ (high confidence), extremely unlikely less than $1^{\circ} \mathrm{C}$ (high confidence), and very unlikely greater than $6^{\circ} \mathrm{C}$ (medium confidence)' (Box 12.2 in Collins et al., 2013). The Charney report began the process of convergence of opinion around a single probability range (essentially, category (2) in the definition of deep uncertainty, above), at least for sensitivity arising from fast feedbacks captured by global climate models (Hansen et al., 2007). Subsequent assessments increased confidence, eliminating deep uncertainty about this part of the sensitivity problem over a wide range of probability.

\section{Cases of Deep Uncertainty from SROCC}

Case A: Permafrost carbon and greenhouse gas emissions. AR5 reported the estimated size of the organic carbon pool stored frozen in permafrost zone soils, but uncertainty estimates were not available (Tarnocai et al., 2009; Ciais et al., 2013). AR5 further reported that future greenhouse gas emissions $\left(\mathrm{CO}_{2}\right.$ only) from permafrost were the most uncertain biogeochemical feedback on climate of the ten factors quantified (Figure 6.20 in Ciais et al., 2013). However, the low confidence assigned to permafrost was not due to few studies, but rather to divergence on the conceptual framework relating changes in permafrost carbon and future greenhouse gas emissions, as well as the probability distribution of key variables. Most large-scale carbon climate models still lack key landscape-level mechanisms that are known to abruptly thaw permafrost and expose organic carbon to decomposition, and many do not include mechanisms needed to differentiate the release of methane versus carbon dioxide with their very different global warming potentials. Studies since AR5 on potential methane release from laboratory soil incubations (Schädel et al., 2016; Knoblauch et al., 2018), actual methane release from the Siberian shallow Arctic ocean shelves (Shakhova et al., 2013; Thornton et al., 2016), changes in permafrost carbon stocks from the Last Glacial Maximum until present (Ciais et al., 2011; Lindgren et al., 2018) and potential carbon uptake by future plant growth (Qian et al., 2010; McGuire et al., 2018) have widened rather than narrowed the uncertainty range (Section 3.4.3.1.1). Accounting for greenhouse gas release from polar and high mountain (Box 2.2) permafrost, introduces an element of deep uncertainty when determining emissions pathways consistent with Article 2 of the Paris Agreement (Comyn-Platt et al., 2018). With stakeholder needs in mind, scientists have been actively engaged in narrowing this uncertainty by using multiple lines of evidence, expert judgment, and joint evaluation of observations and models. As a result, SROCC has reduced uncertainty and introduced confidence assessments across some but not all components of this problem (Section 3.4.3.1.1.).

Case B: Antarctic ice sheet and sea level rise. Dynamical ice loss from Antarctica (Cross-Chapter Box 8 in Chapter 3) provides an example of lack of knowledge about processes, and disagreement about appropriate models and probability distributions for representing uncertainty (categories (1) and (2) in the definition of deep uncertainty). AR5 used a statistical model and expert judgment to reduce uncertainty compared to AR4 (Church et al., 2013). Based on modelling of marine ice sheet processes after AR5, SROCC has further reduced uncertainty in the Antarctic contribution to sea level rise. The likely range including the potential contribution of marine ice sheet instability is quantified as $0.02-0.23 \mathrm{~m}$ for 2081-2100 (and 0.03-0.28 m for 2100) compared to 1986-2005 under RCP8.5 (medium confidence). However, the magnitude of additional rise beyond 2100, and the probability of greater sea level rise than that included in the likely range before 2100 , are characterised by deep uncertainty (Section 4.2.3). 
Cross-Chapter Box 5 (continued)

Policy makers at various levels of governance are considering adaptation investments (e.g., hard infrastructure, retreat, and nature-based defences) for multi-decadal time horizons that consider projection uncertainty (Sections 4.4.2, 4.4.3). For example, extreme sea levels (e.g., the local 'hundred-year flood') now occurring during storms that are historically rare are projected to become annual events by 2100 or sooner at many low-lying coastal locations (Section 4.4.3). Sea level rise exceeding the likely range, or an alternate pathway to the assumed climate change scenario (e.g., which RCP is used in risk estimation), could alter these projections and both factors are characterised by deep uncertainty. Among the strategies used to reduce deep uncertainty in these cases are formal and informal elicitation of expert judgment to project ice sheet behaviour (Horton et al., 2014; Bamber et al., 2019), and development of plausible sea level rise scenarios, including extreme cases (Sections 4.2.3, 4.4.5.3). Frameworks for risk management under deep uncertainty in the context of time lags between commitment to ice sheet losses and emissions mitigation, and between coastal adaptation planning and implementation, are currently emerging in the literature (Section 4.4.5.3.4).

Case C: Compound risks and cascading impacts. Compound risks and cascading impacts (Section 6.1, 6.8, Figure 1.1, Figure 6.1) arise from multiple coincident or sequential hazards (Zscheischler et al., 2018). Compound risks are an example of deep uncertainty because their rarity means that there is often a lack of data or modelling to characterise the risks statistically under present conditions or future changes (Gallina et al., 2016), and there is the potential that climate elements could cross tipping points (e.g., Cai et al., 2016). Nevertheless, effective risk reduction strategies can be developed without knowing the statistical likelihoods of such events by acknowledging the possibility that an event can occur (Dessai et al., 2009). Such strategies are typically well hedged against a variety of different futures and adjustable through time in response to emerging information (Lempert et al., 2010). Case studies are useful for raising awareness of the possibility of compound events and provide valuable learnings for decision makers in the form of analogues (McLeman and Hunter, 2010). They can provide a basis for devising scenarios to stress test systems in other regions for the purposes of understanding and reducing risk. The case study describing the ocean, climate and weather events in the Australian state of Tasmania in 2015/2016 (Box 6.1) provides such an example. It led to compound risks that could not have been estimated due to deep uncertainty. The total cost of the co-occurring fires, floods and marine heat wave to the state government was estimated at about 300 million USD, and impacts on the food, energy and manufacturing sectors reduced Tasmania's anticipated economic growth by approximately half (Eslake, 2016). In the aftermath of this event, the government increased funding to relevant agencies responsible for flood and bushfire management and independent reviews have recommended major policy reforms that are now under consideration (Blake et al., 2017; Tasmanian Climate Change Office, 2017).

\section{What can we learn from SROCC cases in addressing deep uncertainty?}

Using the adapted definition as a framing concept for deep uncertainty (see also Annex 1: Glossary), we find that each of the three cases described in this Cross-Chapter Box involve at least one of the three ways that deep uncertainty can manifest. In Case A, incomplete knowledge on relationships and key drivers and feedbacks (category 1), coupled with broadened probability distributions in post-AR5 literature (category 2), are key reasons for deep uncertainty. In Case B, the inability to characterise the probability of marine ice sheet instability due to a lack of adequate models resulting in divergent views on the probability of ice loss lead to deep uncertainty (categories 1 and 2). In Case C, the Australian example provides insights on the inadequacy of models or previous experience for estimating risk of multiple simultaneous extreme events, contributing to the exhaustion of resources which were then insufficient to meet the need for emergency response. This case also points to the complex task of addressing multiple simultaneous extreme events, and the multiple ways of valuing preferred outcomes in reducing future losses (category 3 ).

The three cases validate the continued iterative process required to meaningfully engage with deep uncertainty in situations of risk, through means such as elicitation, deliberation and application of expert judgement, scenario building and invoking multiple lines of evidence. These approaches demonstrate feasible ways to address or even reduce deep uncertainty in complex decision situations (see also Marchau et al., 2019a), considering that possible obstacles and time investment needed to address deep uncertainty, should not be underestimated. 


\subsection{Integrated Storyline of this Special Report}

The chapters that follow in this special report are framed around geographies or climatic processes where the ocean and/or cryosphere are particularly important for ecosystems and people. The chapter order follows the movement of water from Earth's shrinking mountain and polar cryosphere into our rising and warming ocean.

Chapter 2 assesses High Mountain areas outside of the polar regions, where glaciers, snow and/or permafrost are common. Chapter 3 moves to the Polar Regions of the northern and southern high latitudes, which are characterised by vast stores of frozen water in ice sheets, glaciers, ice shelves, sea ice and permafrost, and by the interaction of these cryosphere elements and the polar oceans. Chapter 4 examines Sea Level Rise and the hazards this brings to Low-Lying Regions, Coasts and Communities. Chapter 5 focuses on the Changing Ocean, with a particular focus on how climate change impacts on the ocean are altering Marine Ecosystems and affecting Dependent Communities. Chapter 6 is dedicated to assessing Extremes and Abrupt Events, and reflects the potential for rapid and possibly irreversible changes in Earth's ocean and cryosphere, and the challenges this brings to Managing Risk. The multitude ways in which Low-Lying Islands and Coasts are exposed and vulnerable to the impacts of ocean and cryosphere change, along with resilience and adaptation strategies, opportunities and governance options specific to these settings, is highlighted in integrative Cross-Chapter Box 9.
This report does not attempt to assess all aspects of the ocean and cryosphere in a changing climate. Examples of research themes that will be covered elsewhere in the IPCC Sixth Assessment Cycle and not SROCC include: assessments of ocean and cryosphere changes in the CMIP6 experiments (AR6), cryosphere changes outside of polar and high mountain regions (e.g., snow cover in temperate and low altitude settings; AR6), and a thorough assessment of mitigation options for reducing climate change impacts (SR15, AR6 WGIII).

Each chapter of SROCC presents an integrated storyline on the ocean and/or cryosphere in a changing climate. The chapter assessments each present evidence of the pervasive changes that are already underway in the ocean and cryosphere (Figure 1.5). The impacts that physical changes in the ocean and cryosphere have had on ecosystems and people are assessed, along with lessons learned from adaptation measures that have already been employed to avoid adverse impacts. The assessments of future change in the ocean and cryosphere demonstrate the growing and accelerating changes projected for the future and identify the reduced impacts and risks that choices for a low greenhouse gas emission future would have compared with a high emission future (Figure 1.5). Potential adaptation strategies to reduce future risks to ecosystems and people are assessed, including identifying where limits to adaptation may be exceeded. The local- to global-scale responses for charting CRDPs are also assessed.

Figure 1.5 (right) Observed and modelled historical changes in the ocean and cryosphere since 1950, and projected future changes under low (RCP2.6) and high (RCP8.5) greenhouse gas emissions scenarios (Cross-Chapter Box 1 in Chapter 1). -Changes are shown for: (a) Global mean surface air temperature change with likely range (Cross-Chapter Box 1 in Chapter 1). Ocean-related changes with very likely ranges for (b) Global mean sea surface temperature change (Box 5.1, Section 5.2.2); (c) Change factor in surface ocean marine heatwave days (6.4.1); (d) Global ocean heat content change (0-2000 $\mathrm{m}$ depth). An approximate steric sea level equivalent is shown with the right axis by multiplying the ocean heat content by the global-mean thermal expansion coefficient $\left(\varepsilon \approx 0.125 \mathrm{~m}\right.$ per $10^{24}$ Joules) for observed warming since 1970 (Figure 5.1 ); (h) Global mean surface $\mathrm{pH}$ (on the total scale). Assessed observational trends are compiled from open ocean time series sites longer than 15 years (Box 5.1, Figure 5.6, Section 5.2.2); and (i) Global mean ocean oxygen change (100-600 m depth). Assessed observational trends span 1970-2010 centered on 1996 (Figure 5.8, Section 5.2.2). Sea-level changes with likely ranges for $(\mathbf{m})$ Global mean sea level change. Hashed shading reflects low confidence in sea level projections beyond 2100 and bars at 2300 reflect expert elicitation on the range of possible sea level change (Section 4.2.3, Figure 4.2); and components from (e,f) Greenland and Antarctic ice sheet mass loss (Section 3.3.1); and (g) Glacier mass loss (Cross-Chapter Box 6 in Chapter 2, Table 4.1). Further cryosphere-related changes with very likely ranges for (j) Arctic sea ice extent change for September (Sections 3.2.1, 3.2.2 Figure 3.3); (k) Arctic snow cover change for June (land areas north of 60 $\mathrm{N}$ ) (Sections 3.4.1, 3.4.2, Figure 3.10); and (I) Change in near-surface (within 3-4 m) permafrost area in the Northern Hemisphere (Sections 3.4.1, 3.4.2, Figure 3.10). 
Past and future changes in the ocean and cryosphere

Historical changes (observed and modelled) and projections under RCP2.6 and RCP8.5 for key indicators

- Historical (observed) H Historical (modelled)
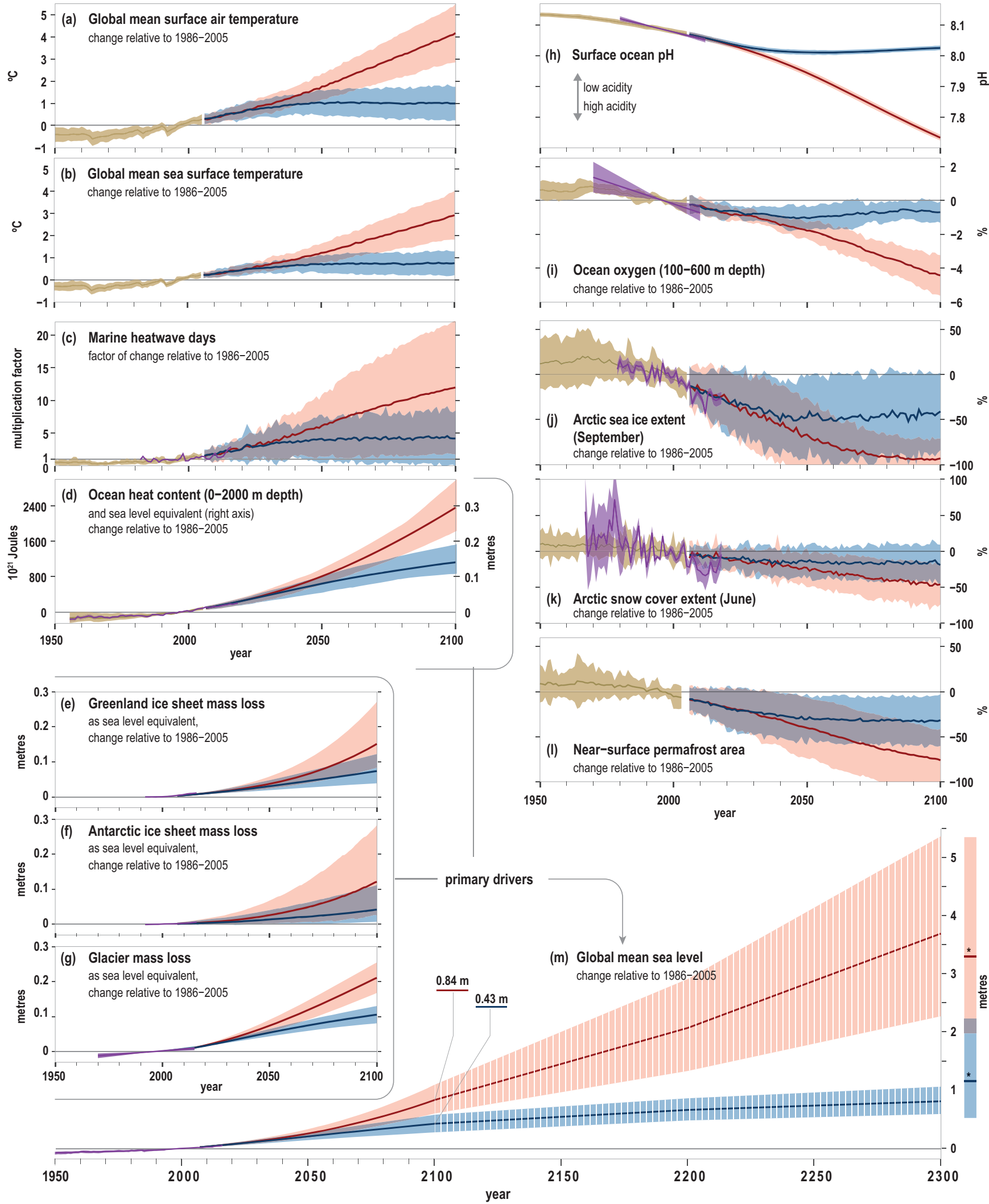

Projected (RCP2.6)

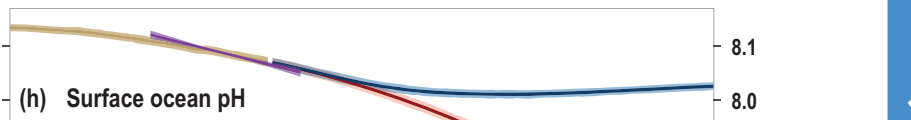

(i) Ocean oxygen (100-600 m depth) change relative to $1986-2005$

(i) Arctic sea ice extent (September)
change relative to 1986-2005
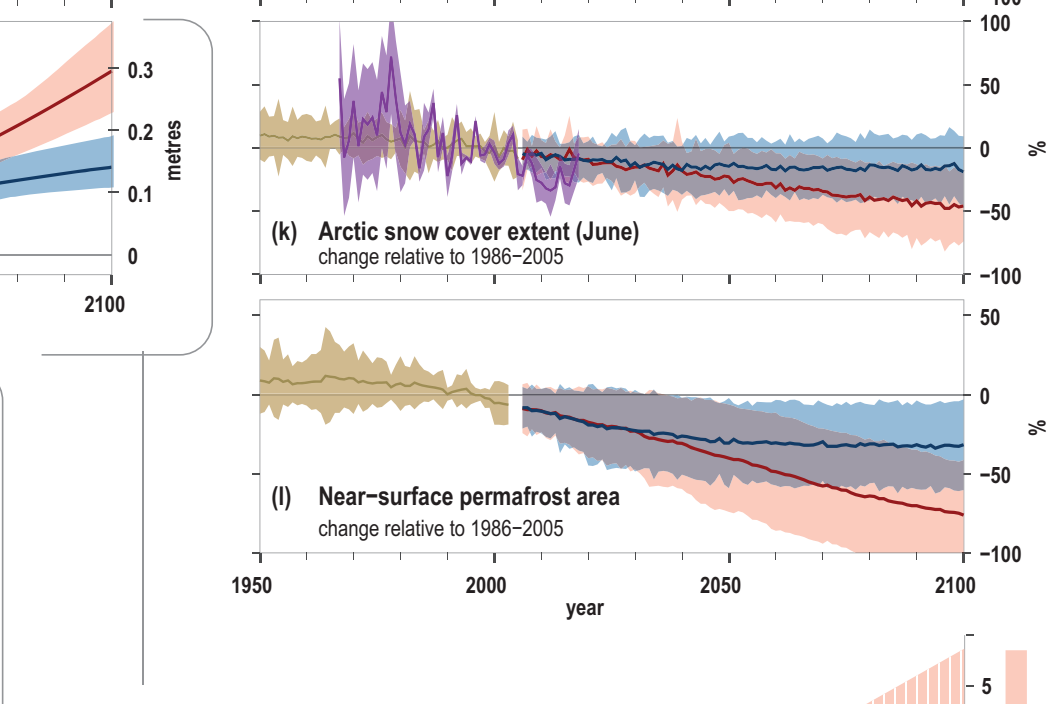

primary drivers

(m) Global mean sea level

$0.84 \mathrm{~m}$ change relative to $1986-2005$ 
Frequently Asked Questions

\section{FAQ 1.1 How do changes in the ocean and cryosphere affect our life on planet Earth?}

The ocean and cryosphere regulate the climate and weather on Earth, provide food and water, support economies, trade and transportation, shape cultures and influence our well-being. Many of the recent changes in Earth's ocean and cryosphere are the result of human activities and have consequences on everyone's life. Deep cuts in greenhouse gas emissions will reduce negative impacts on billions of people and help them adapt to changes in their environment. Improving education and combining scientific knowledge with Indigenous knowledge and local knowledge helps communities to further address the challenges ahead.

The ocean and cryosphere - a collective name for the frozen parts of the Earth - are essential to the climate and life giving processes on our planet.

Changes in the ocean and cryosphere occur naturally, but the speed, magnitude, and pervasiveness of the global changes happening right now have not been observed for millennia or longer. Evidence shows that the majority of ocean and cryosphere changes observed in the past few decades are the result of human influences on Earth's climate.

Every one of us benefits from the role of the ocean and cryosphere in regulating climate and weather. The ocean has absorbed about a third of the carbon dioxide humans have emitted from the burning of fossil fuels since the Industrial Revolution, and the majority (more than 90\%) of the extra heat within the Earth system. In this way, the ocean has slowed the warming humans and ecosystems have experienced on land. The reflective surface of snow and ice reduce the amount of the sun's energy that is absorbed on Earth. This effect diminishes as snow and ice melts, contributing to amplified temperature rise across the Arctic. The ocean and cryosphere also sustain life giving water resources, by rain and snow that come from the ocean, and by melt water from snow and glaciers in mountain and polar regions.

Nearly two billion people live near the coast, and around 800 million on land less than $10 \mathrm{~m}$ above sea level. The ocean directly supports the food, economies, cultures and well-being of coastal populations (see FAQ 1.2). The livelihoods of many more are tied closely to the ocean through food, trade, and transportation. Fish and shellfish contribute about $17 \%$ of the non-grain protein in human diets and shipping transports at least $80 \%$ of international imports and exports. But the ocean also brings hazards to coastal populations and infrastructure, and particularly to low-lying coasts. These populations are increasingly exposed to tropical cyclones, marine heat waves, sea level rise, coastal flooding and saltwater incursion into groundwater resources.

In high mountains and the Arctic, around 700 million people live in close contact with the cryosphere. These people, including many Indigenous Peoples, depend on snow, glaciers and sea ice for their livelihoods, food and water security, travel and transport, and cultures (see FAQ 1.2). They are also exposed to hazards as the cryosphere changes, including flood outbursts, landslides and coastal erosion. Changes in the polar and high mountain regions also have far-reaching consequences for people in other parts of the world (see FAQ 3.1).

Warming of the climate system leads to sea level rise. Melt from glaciers and ice sheets is adding to the amount of water in the ocean, and the heat being absorbed by the ocean is causing it to expand and take up more space. Today's sea level is already about $20 \mathrm{~cm}$ higher than in 1900 . Sea level will continue to rise for centuries to millennia because the ocean system reacts slowly. Even if global warming were to be halted, it would take centuries or more to halt ice sheet melt and ocean warming.

Enhanced warming in the Arctic and in high mountains is causing rapid surface melt of glaciers and the Greenland ice sheet. Thawing of permafrost is destabilising soils, human infrastructure, and Arctic coasts, and has the potential to release vast quantities of methane and carbon dioxide into the atmosphere that will further exacerbate climate change. Widespread loss of sea ice in the Arctic is opening up new routes for shipping, but at the same time is reducing habitats for key species and affecting the livelihoods of Indigenous cultures. In Antarctica, glacier and ice sheet loss is occurring particularly quickly in places where ice is in direct contact with warm ocean water, further contributing to sea level rise.

Ocean ecosystems are threatened globally by three major climate change-induced stressors: warming, loss of oxygen and acidification. Marine heat waves are occurring everywhere across the surface ocean, and are becoming more frequent and more intense as the ocean warms. These are causing disease and mass-mortality 
that put, for example, coral reefs and fish populations at risk. Marine heat waves last much longer than the heat waves experienced on land, and are particularly harmful for organisms that cannot move away from areas of warm water.

Warming of the ocean reduces not only the amount of oxygen it can hold, but also tend to stratify it. As a result, less oxygen is transported to depth, where it is needed to support ocean life. Dissolved carbon dioxide that has been taken up by the ocean reacts with water molecules to increase the acidity of seawater. This makes the water more corrosive for marine organisms that build their shells and structures out of mineral carbonates, such as corals, shellfish and plankton. These climate-change stressors occur alongside other human-driven impacts, such as overfishing, excessive nutrient loads (eutrophication), and plastic pollution. If human impacts on the ocean continue unabated, declines in ocean health and services are projected to cost the global economy 428 billion USD $\mathrm{yr}^{-1}$ by 2050, and 1.979 trillion USD $\mathrm{yr}^{-1}$ by 2100 .

The speed and intensity of the future risks and impacts from ocean and cryosphere change depend critically on future greenhouse gas emissions. The more these emissions can be curbed, the more the changes in the ocean and cryosphere can be slowed and limited, reducing future risks and impacts. But humankind is also exposed to the effects of changes triggered by past emissions, including sea level rise that will continue for centuries to come. Improving education and using scientific knowledge alongside local knowledge and Indigenous knowledge can support the development of context-specific options that help communities to adapt to inevitable changes and respond to challenges ahead. 


\section{FAQ 1.2 How will changes in the ocean and cryosphere affect meeting the Sustainable Development Goals?}

Ocean and cryosphere change affect our ability to meet the United Nations Sustainable Development Goals (SDGs). Progress on the SDGs support climate action that will reduce future ocean and cryosphere change, and as well as the adaptation responses to unavoidable changes. There are also trade-offs between SDGs and measures that help communities to adjust to their changing environment, but limiting greenhouse gas emissions opens more options for effective adaptation and sustainable development.

The SDGs were adopted by the United Nations in 2015 to support action for people, planet and prosperity (FAQ 1.2, Figure 1). The 17 goals and their 169 targets strive to end poverty and hunger, protect the planet and reduce gender, social and economic inequities by 2030.

SDG 13 (Climate Action) explicitly recognises that changing climatic conditions are a global concern. Climate change is already causing pervasive changes in Earth's ocean and cryosphere (FAQ 1.1). These changes are impacting food, water and health securities, with consequences for achieving SDG 2 (Zero Hunger), SDG 3 (Good Health and Well-Being), SDG 6 (Clean Water and Sanitation), and SDG 1 (No Poverty). Climate change impacts on Earth's ocean and cryosphere also affect the environmental goals for SDG 14 (Life below Water) and SDG 15 (Life on Land), with additional implications for many of the other SDGs.

SDG 6 (Clean Water and Sanitation) will be affected by ocean and cryosphere changes. Melting mountain glaciers bring an initial increase in water, but as glaciers continue to shrink so too will the essential water they provide to millions of mountain dwellers, downstream communities, and cities. These populations also depend on water flow from the high mountains for drinking, sanitation, and irrigation, and for SDG 7 (Affordable and Clean Energy). Water security is also threatened by changes in the magnitude and seasonality of rainfall, driven by rising ocean temperatures, which increases the risk of severe storms and flooding in some regions, or the risk of more severe or more frequent droughts in other regions. Among other effects, ongoing sea level rise is allowing salt water to intrude further inland, contaminating drinking water and irrigation sources for some coastal populations. Actions to address these threats will likely require new infrastructure to manage rain, melt water, and river flow, in order to make water supplies more reliable. These actions would also benefit SDG 3 (Good Health and Well-Being) by reducing the risk of flooding and negative health outcomes posed by extreme rainfall and outbursts of glacial melt.

Climate change impacts on the ocean and cryosphere also have many implications for progress on food security that is addressed in SDG 2 (Zero Hunger). Changes in rainfall patterns caused by ocean warming will increase aridity in some areas and bring more (or more intense) rainfall to others. In mountain regions, these changes bring varying challenges for maintaining reliable crops and livestock production. Some adaptation opportunities might be found in developing strains of crops and livestock better adapted to the future climate conditions, but this response option is also challenged by the rapid rate of climate change. In the Arctic, very rapidly warming temperatures, diminishing sea ice, reduced snow cover and degradation of permafrost are restricting the habitats and migration patterns of important food sources (SDG 2 Zero Hunger), including reindeer and several marine mammals (SDG 15 Life on Land; SDG 14 Life below Water), resulting in reduced hunting opportunities for staple foods that many northern Indigenous communities depend upon.

Rising temperatures, and changes in ocean nutrients, acidity and salinity are altering SDG 14 (Life Below Water). The productivity and distributions of some fish species are changing in ways that alter availability of fish to long-established fisheries, whereas the range of fish populations may move to become available in some new coastal and open ocean areas.

Ocean changes are of concern for small island developing states and coastal cities and communities. Beyond possible reductions in marine food supply and related risks for SDG 2 (Zero Hunger), their lives, livelihoods and well-being are also threatened in ways that are linked to several SDGs, including SDG 3 (Good Health and Wellbeing), SDG 8 (Decent Work and Economic Growth), SDG 9 (Industry, Innovation, and Infrastructure), and SDG 11 (Sustainable Cities and Communities). For example, sea level rise and warming oceans can cause inundation of coastal homes and infrastructure, more powerful tropical storms, declines in established economies such as tourism and losses of cultural heritage and identity. Improved community and coastal infrastructure can help to adapt to these changes, and more effective and faster disaster responses from health sectors and other emergency services can assist the populations who experience these impacts. In some situations, the most appropriate responses may involve relocation of critical services and, in some cases, communities; and for some populations, migration away from their homeland may become the only viable response. 


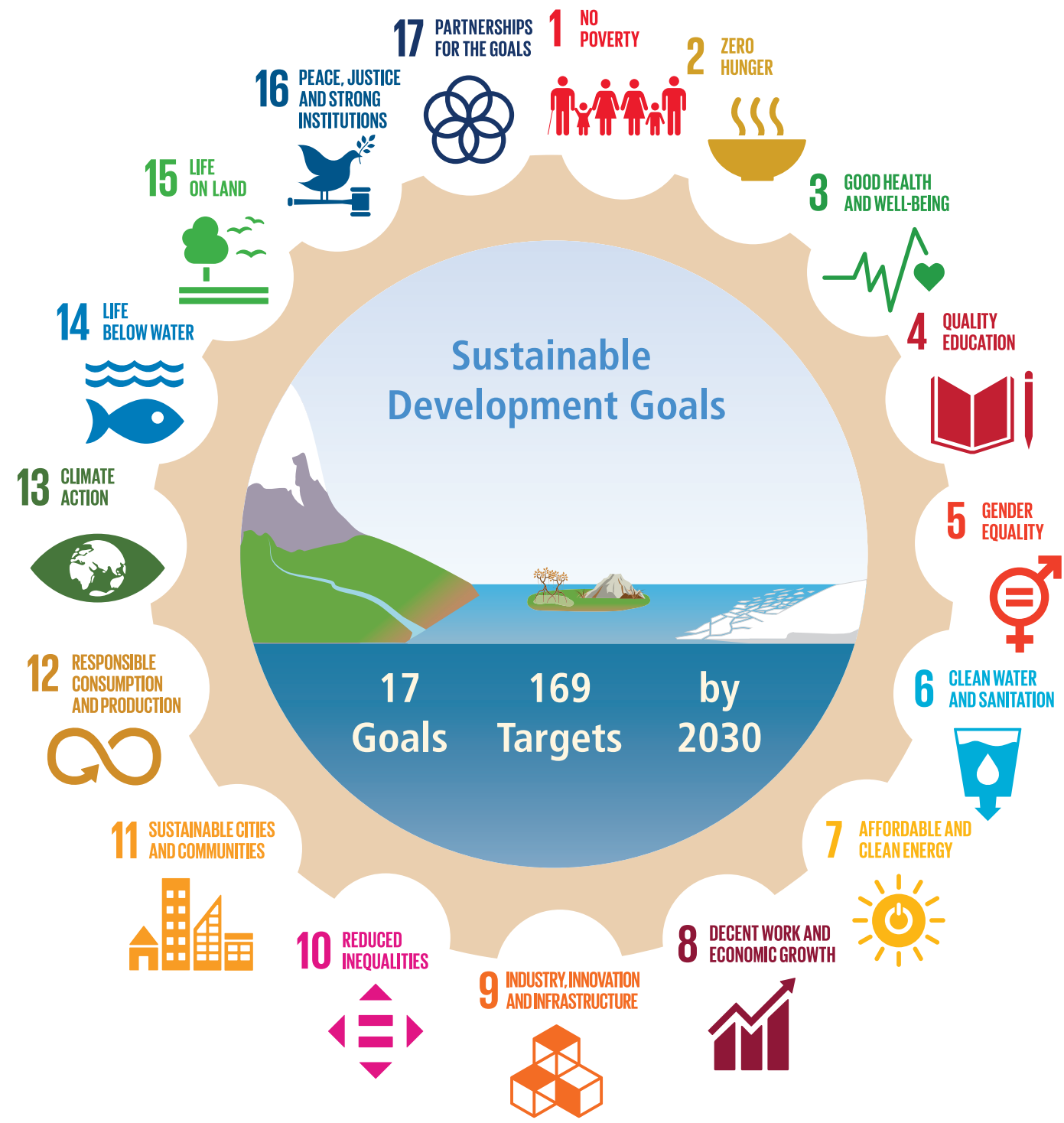

FAQ 1.2, Figure 1 | The United Nations 2030 Sustainable Development Goals (SDGs).

Without transformative adaptation and mitigation, climate change could undermine progress towards achieving the 2030 SDGs, and make it more difficult to implement CRDPs in the longer term. Reducing global warming (mitigation) provides the best possibility to limit the speed and extent of ocean and cryosphere change and give more options for effective adaptation and sustainable development. Progress on SDG 4 (Quality Education), SDG 5 (Gender Equality) and SDG 10 (Reduced Inequalities) can moderate the vulnerabilities that shape people's risk to ocean and cryosphere change, while SDG 12 (Responsible Consumption and Production), SDG 16 (Peace, Justice and Institutions) and SDG 17 (Partnerships for the Goals) will help to facilitate the scales of adaptation and mitigation responses required to achieve sustainable development. Investment in social and physical infrastructure that supports adaptation to inevitable ocean and cryosphere changes will enable people to participate in initiatives to achieve the SDGs. Current and past IPCC efforts have focused on identifying CRDPs. Such adaptation and mitigation strategies, supported by adequate investments, and understanding the potential for SDG initiatives to increase the exposure or vulnerability of the activities to climate change hazards, could also constitute pathways for progress on the SDGs. 


\section{Acknowledgements}

Thanks are due to Mohd Abdul Fahad, Mohamed Khamla, Jelto von Schuckmann and Debabrat Sukla for their assistance with early versions of figures and graphics.

\section{References}

Abraham, J.P. et al., 2013: A review of global ocean temperature observations: Implications for ocean heat content estimates and climate change. Rev. Geophysics, 51(3), 450-483, doi:10.1002/rog.20022.

Abram, N.J. et al., 2016: Early onset of industrial-era warming across the oceans and continents. Nature, 536(7617), 411-418, doi:10.1038/ nature19082.

Abram, N.J. et al., 2013: Acceleration of snow melt in an Antarctic Peninsula ice core during the twentieth century. Nat. Geosci., 6(5), 404-411, doi:10.1038/ngeo1787.

Acharya, A. and A. Prakash, 2018: When the river talks to its people: local knowledge-based flood forecasting in Gandak River basin, India. Environmental Development, 31, 55-67, doi:10.1016/j. envdev.2018.12.003.

Ackerman, F., 2013: Valuing the ocean environment. In: Managing ocean environments in a changing climate: sustainability and economic perspectives [Noone, K.J., U.R. Sumaila and R.J. Diaz (eds.)]. Elsevier, Amsterdam, pp. 243-275. ISBN 9780124076686.

Adger, W.N. et al., 2013: Cultural dimensions of climate change impacts and adaptation. Nat. Clim. Change, 3(2), 112-117, doi:10.1038/Nclimate1666.

Adger, W.N. et al., 2009: Are there social limits to adaptation to climate change? Clim. Change, 93(3-4), 335-354, doi:10.1007/s10584-008-9520-z.

Adger, W.N. et al., 2014: Human security. In: Climate Change 2014: Impacts, Adaptation, and Vulnerability. Part A: Global and Sectoral Aspects. Contribution of Working Group II to the Fifth Assessment Report of the Intergovernmental Panel on Climate Change [Field, C.B., V.R. Barros, D.J. Dokken, K.J. Mach, M.D. Mastrandrea, T.E. Biller, M. Chatterjee, K.L. Ebi, Y.O. Estrada, R.C. Genova, B. Girma, E.S. Kissel, A.N. Levy, S. MacCracken, P.R. Mastrandrea and L.L. White (eds.)]. Cambridge University Press, Cambridge, United Kingdom and New York, NY, USA, 793-832.

Adler, C.E. and G. Hirsch Hadorn, 2014: The IPCC and treatment of uncertainties: Topics and sources of dissensus. WIREs. Clim. Change, 5(5), 663-676, doi:10.1002/wcc.297.

Agrawal, A., 1995: Dismantling the divide between indigenous and scientific knowledge. Dev. Change, 26(3), 413-439, doi:10.1111/j.1467-7660.1995. tb00560.x.

Albrecht, G. et al., 2007: Solastalgia: the distress caused by environmental change. Australas. Psych., 15, S95-S98, doi:10.1080/10398560701701288.

Allen, M.R. et al., 2018: Framing and Context. In: Global Warming of $1.5^{\circ} \mathrm{C}$. An IPCC Special Report on the impacts of global warming of $1.5^{\circ} \mathrm{C}$ above pre-industrial levels and related global greenhouse gas emission pathways, in the context of strengthening the global response to the threat of climate change, sustainable development, and efforts to eradicate poverty [Masson-Delmotte, V., P. Zhai, H.-O. Portner, D. Roberts, J. Skea, P.R. Shukla, A. Pirani, W. Moufouma-Okia, C. Péan, R. Pidcock, S. Connors, J.B.R. Matthews, Y. Chen, X. Zhou, M.I. Gomis, E. Lonnoy, T. Maycock, M. Tignor and T. Waterfield (eds.)]. World Meteorological Organization, Geneva, Switzerland, 49-91.

AMAP, 2011: Snow, Water, Ice and Permafrost in the Arctic (SWIPA): Climate Change and the Cryosphere. Arctic Monitoring and Assessment Programme (AMAP), Oslo, Norway, $538 \mathrm{pp}$.

AMAP, 2015: AMAP Assessment 2015: Human Health in the Arctic. Arctic Monitoring and Assessment Programme (AMAP), Oslo, Norway, 165 pp.
AMAP, 2017: Snow, Water, Ice and Permafrost in the Arctic (SWIPA) 2017. Arctic Monitoring and Assessment Programme (AMAP), Oslo, Norway, 269 pp.

Amsler, L.B., 2016: Collaborative governance: Integrating management, politics, and law. Public Adm. Rev., 76(5), 700-711, doi:10.1111/ puar.12605.

Amundsen, H. et al., 2018: Local governments as drivers for societal transformation: Towards the $1.5^{\circ} \mathrm{C}$ ambition. Curr. Opin. Environ. Sustain., 31, 23-29, doi:10.1016/j.cosust.2017.12.004.

Andrade, E. et al., 2018: Project Report: Ascertainment of the estimated excess mortality from Hurricane Maria in Puerto Rico. Milken Institute School of Public Health of The George Washington University, Washington D.C., $69 \mathrm{pp}$.

Andreone, G., 2017: The Future of the Law of the Sea: Bridging Gaps Between National, Individual and Common Interests. Springer International Publishing, Cham, Switzerland. ISBN 9783319512747.

Anisimov, 0.A. and R. Orttung, 2018: Climate change in Northern Russia through the prism of public perception. Ambio, 48(6), 661-671, doi:10.1007/s13280-018-1096-x.

Armitage, D. et al., 2011: Co-management and the co-production of knowledge: Learning to adapt in Canada's Arctic. Global Environ. Change, 21(3), 995-1004, doi:10.1016/j.gloenvcha.2011.04.006.

Arrigo, K.R. et al., 2017: Melting glaciers stimulate large summer phytoplankton blooms in southwest Greenland waters. Geophys. Res. Letters, 44(12), 6278-6285, doi:10.1002/2017GL073583.

Ayers, J. and T. Forsyth, 2009: Community-Based adaptation to climate change. Environment: Science and Policy for Sustainable Development, 51(4), 22-31, doi:10.1093/acrefore/9780190228620.013.602.

Baker, B. and B. Yeager, 2015: Coordinated ocean stewardship in the Arctic: Needs, challenges and possible models for an Arctic Ocean coordinating agreement. Transnational Environmental Law, 4(2), 359-394, doi:10.1017/ S2047102515000151.

Baldwin, E., P. McCord, J. Dell'Angelo and T. Evans, 2018: Collective action in a polycentric water governance system. Environmental Policy and Governance, 28(4), 212-222, doi:10.1002/eet.1810.

Balmaseda, M.A. et al., 2015: The Ocean Reanalyses Intercomparison Project (ORA-IP). J. Oper. Oceanogr., 8(sup1), s80-s97, doi:10.1080/175587 6X.2015.1022329.

Balmaseda, M.A., K.E. Trenberth and E. Källén, 2013: Distinctive climate signals in reanalysis of global ocean heat content. Geophys. Res. Letters, 40(9), 1754-1759, doi:10.1002/grl.50382.

Bamber, J.W. et al., 2019: Ice sheet contributions to future sea level rise from structured expert judgement. Proc. Natl. Acad. Sci. U.S.A. 116(23), 1119511200, doi:10.1073/pnas.1817205116.

Baptiste, B., D. Pacheco, M. Carneiro da Cunha and S. Diaz, 2017: Knowing our lands and resources: Indigenous and local knowledge of biodiversity and ecosystem services in the Americas. Knowledges of Nature 11, UNESCO, Paris, 176 pp.

Barange, M. et al., 2014: Impacts of climate change on marine ecosystem production in societies dependent on fisheries. Nat. Clim. Change, 4(3), 211-216, doi:10.1038/nclimate2119. 
Barber, D. et al., 2001: Physical processes within the North Water (NOW) polynya. Atmos. Ocean, 39(3), 163-166, doi:10.1080/07055900.2001.96 49673.

Barnett, J. et al., 2014: A local coastal adaptation pathway. Nat. Clim. Change, 4(12), 1103-1108, doi:10.1038/nclimate2383.

Bartlett, C., M. Marshall and A. Marshall, 2012: Two-eyed seeing and other lessons learned within a co-learning journey of bringing together indigenous and mainstream knowledges and ways of knowing. J. Environ. Stud. Sci., 2(4), 331-340, doi:10.1007/s13412-012-0086-8.

Beck, S. et al., 2014: Towards a reflexive turn in the governance of global environmental expertise the cases of the IPCC and the IPBES. GAIA., 23(2), 80-87, doi:10.14512/gaia.23.2.4.

Bell, J.D., J.E. Johnson and A.J. Hobday, 2011: Vulnerability of tropical Pacific fisheries and aquaculture to climate change. Secretariat of the Pacific Community, Noumea, New Caledonia, 925 pp.

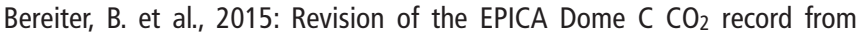
800 to 600 kyr before present. Geophys. Res. Letters, 42(2), 542-549, doi:10.1002/2014GL061957.

Berner, R.A. and Z. Kothavala, 2001: GEOCARB III: A revised model of atmospheric $\mathrm{CO}_{2}$ over Phanerozoic time. Am. J. Sci., 301(2), 182-204, doi:10.2475/ajs.301.2.182.

Berrang-Ford, L. et al., 2014: What drives national adaptation? A global assessment. Clim. Change, 124(1-2), 441-450, doi:10.1007/s10584-0141078-3.

Biemans, H. et al., 2019: Importance of snow and glacier meltwater for agriculture on the Indo-Gangetic Plain. Nature Sustainability, 2, 594-601, doi: 10.1038/s41893-019-0305-3.

Biggs, R. et al., 2012: Toward principles for enhancing the resilience of ecosystem services. Annu. Rev. Environ. Resour., 37(1), 421-448, doi:10.1146/annurev-environ-051211-123836.

Billé, R. et al., 2013: Taking action against ocean acidification: a review of management and policy options. Environ. Manage., 52(4), 761-779, doi:10.1007/s00267-013-0132-7.

Bindoff, N.L. and P.A. Stott, 2013: Detection and Attribution of Climate Change: from Global to Regional. In: Climate Change 2013: The Physical Science Basis. Contribution of Working Group I to the Fifth Assessment Report of the Intergovernmental Panel on Climate Change [Stocker, T.F., D. Qin, G.-K. Plattner, M. Tignor, S.K. Allen, J. Boschung, A. Nauels, Y. Xia, V. Bex and P.M. Midgley (eds.)]. Cambridge University Press, Cambridge, United Kingdom and New York, NY, USA, 867-952.

Bisaro, A. and J. Hinkel, 2016: Governance of social dilemmas in climate change adaptation. Nat. Clim. Change, 6(4), 354-359, doi:10.1038/ nclimate2936.

Black, R., S.R. Bennett, S.M. Thomas and J.R. Beddington, 2011: Climate change: Migration as adaptation. Nature, 478(7370), 447-449, doi:10.1038/478477a.

Blake, M., P. Considine and B. Edmons, 2017: Report of the independent review into the Tasmanian floods of June and July 2016: Shared responsibility, resilience and adaptation. Department of Premier and Cabinet (Tasmania), Hobart, Tasmania, 138 [Available at: www.dpac.tas.gov.au/_data/assets/ pdf_file/0015/332610/floodreview.pdf].

Bloemen, P., M. Van Der Steen and Z. Van Der Wal, 2018: Designing a century ahead: climate change adaptation in the Dutch Delta. Policy and Society, 38(1), 58-76, doi:10.1080/14494035.2018.1513731.

Bojinski, S. et al., 2014: The concept of essential climate variables in support of climate research, applications, and policy. Bull. Am. Meteorol. Soc., 95(9), 1431-1443, doi:10.1175/BAMS-D-13-00047.1.

Bond, N.A., M.F. Cronin, H. Freeland and N. Mantua, 2015: Causes and impacts of the 2014 warm anomaly in the NE Pacific. Geophys. Res. Letters, 42, 3414-3420, doi:10.1002/2015GL063306.

Boyd, E. et al., 2017: A typology of loss and damage perspectives. Nat. Clim. Change, 7(10), 723-729, doi:10.1038/nclimate3389.
Boyd, P.W., S.T. Lennartz, D.M. Glover and S.C. Doney, 2015: Biological ramifications of climate-change-mediated oceanic multi-stressors. Nat. Clim. Change, 5(1), 71-79, doi:10.1038/nclimate2441.

Boyd, P.W., S. Sundby and H.-O. Pörtner, 2014: Cross-chapter box on net primary production in the ocean. In: Climate Change 2014: Impacts, Adaptation, and Vulnerability. Part A: Global and Sectoral Aspects. Contribution of Working Group II to the Fifth Assessment Report of the Intergovernmental Panel on Climate Change [Field, C.B., V.R. Barros, D.J. Dokken, K.J. Mach, M.D. Mastrandrea, T.E. Bilir, M. Chatterjee, K.L. Ebi, Y.O. Estrada, R.C. Genova, B. Girma, E.S. Kissel, A.N. Levy, S. MacCracken, P.R. Mastrandrea and L.L. White (eds.)]. Cambridge University Press, Cambridge, United Kingdom and New York, NY, USA, 133-136.

Boyer, T.P. et al., 2013: World Ocean Database 2013.[Levitus, S. and A. Mishonov (eds.)]. Silver Spring, MD, NOAA Atlas, 209 pp.

Breitburg, D. et al., 2018: Declining oxygen in the global ocean and coastal waters. Science, 359(6371), eaam7240, doi:10.1126/science.aam7240.

Bronen, R., 2015: Climate-induced community relocations: Using integrated social-ecological assessments to foster adaptation and resilience. Ecol. Soc., 20(3), 36, doi:10.5751/ES-07801-200336.

Brügger, A. et al., 2015: Psychological responses to the proximity of climate change. Nat. Clim. Change, 5(12), 1031-1037, doi:10.1038/nclimate2760.

Brysse, K., N. Oreskes, J. O'Reilly and M. Oppenheimer, 2013: Climate change prediction: Erring on the side of least drama? Global Environ. Change, 23(1), 327-337, doi:10.1016/j.gloenvcha.2012.10.008.

Burkett, M., 2016: Reading between the red lines: Loss and damage and the Paris outcome. Climate Law, 6(1-2), 118-129, doi:10.1163/1878656100601008 .

Burnham, M., Z. Ma and B. Zhang, 2016: Making sense of climate change: Hybrid epistemologies, socio-natural assemblages and smallholder knowledge. Area, 48(1), 18-26, doi:10.1111/area.12150.

Butler, J. et al., 2014: Framing the application of adaptation pathways for rural livelihoods and global change in eastern Indonesian islands. Global Environ. Change, 28, 368-382, doi:10.1016/j.gloenvcha.2013.12.004.

Cai, Y., T.M. Lenton and T.S. Lontzek, 2016: Risk of multiple interacting tipping points should encourage rapid $\mathrm{CO}_{2}$ emission reduction. Nat. Clim. Change, 6(5), 520-525, doi:10.1038/nclimate2964.

Cardona, O.D. et al., 2012: Determinants of risk: Exposure and vulnerability. In: Managing the risks of extreme events and disasters to advance climate change adaptation - A special report of Working Groups I and II of the Intergovernmental Panel on Climate Change (IPCC) [Field, C.B., V. Barros, T.F. Stocker, D. Qin, D.J. Dokken, K.L. Ebi, M.D. Mastrandrea, K.J. Mach, G.-K. Plattner, S.K. Allen, M. Tignor and P.M. Midgley (eds.)]. Cambridge University Press, Cambridge, United Kingdom, 65-108.

Carey, M. et al., 2015: Integrated approaches to adaptation and disaster risk reduction in dynamic socio-cryospheric systems. In: Snow and ice-related hazards, risks and disasters [Shroder, J.F., C. Whiteman and W. Haeberli (eds.)]. Elsevier, pp. 219-261. ISBN 9780123948496.

Carlisle, K. and R.L. Gruby, 2017: Polycentric systems of governance: a theoretical model for the commons. Policy Stud. J., 26, doi:10.1111/ psj.12212.

Carpenter, S.R. and W.A. Brock, 2008: Adaptive capacity and traps. Ecol. Soc., 13(2), 40.

Carton, J.A., G.A. Chepurin and L. Chen, 2018: SODA3: A new ocean climate reanalysis. J. Clim, 31, 6967-6983, doi:10.1175/JCLI-D-18-0149.1.

Cassotta, S., 2012: Environmental Damage Liability Problems in a Multilevel Context: The Case of the Environmental Liability Directive. Energy and environmental law \& policy series: Supanational and comparative aspects, Kluwer Law International, 280 pp.

Cassotta, S. and M. Mazza, 2015: Balancing de jure and de facto Arctic environmental law applied to the oil and gas industry: Linking indigenous rights, social impact assessment and business in Greenland. The Yearbook of Polar Law Online, 6(1), 63-119, doi:10.1163/1876-8814_004. 
Castree, N. et al., 2014: Changing the intellectual climate. Nat. Clim. Change, 4(9), 763-768, doi:10.1038/nclimate2339.

Chandler, D., 2013: Resilience and the autotelic subject: Toward a critique of the societalization of security. Int. Political Sociol., 7(2), 210-226, doi:10.1111/ips.12018.

Chapin, F.S., G.P. Kofinas and C. Folke, 2009: Principles of Ecosystem Stewardship: Resilience-Based Natural Resource Management in a Changing World. Springer-Verlag, New York, 401 pp.

Charlesworth, B., 2009: Effective population size and patterns of molecular evolution and variation. Nat. Rev. Genet., 10(3), 195-205, doi:10.1038/ nrg2526.

Charney, J.G. et al., 1979: Carbon Dioxide and Climate:A Scientific Assessment. Report of an Ad Hoc Study Group on Carbon Dioxide and Climate. National Academy of Sciences, Washington, DC., 22 pp.

Chen, Y., Z. Li, G. Fang and W. Li, 2018: Large hydrological processes changes in the transboundary rivers of Central Asia. J. Geophys. Res. Atmos., 123(10), 5059-5069, doi:10.1029/2017jd028184.

Cheng, L. et al., 2018: Taking the pulse of the planet. Eos, 98, 14-16, doi:10.1029/2017EO081839.

Chilisa, B., 2011: Indigenous Research Methodologies. SAGE Publications, Los Angeles, $368 \mathrm{pp}$.

Chircop, A., S. Coffen-Smout and M.L. McConnell, 2018: Ocean Yearbook 32. Ocean Yearbook, Brill Nijhoff, 381 pp. ISBN: 9789004367005.

Chung Tiam Fook, T., 2017: Transformational processes for community-focused adaptation and social change:A synthesis. Clim. Dev., 9(1), 5-21, doi:10.10 80/17565529.2015.1086294.

Church, J.A. et al., 2013: Sea Level Change. In: Climate Change 2013: The Physical Science Basis. Contribution of Working Group I to the Fifth Assessment Report of the Intergovernmental Panel on Climate Change [Stocker, T.F., D. Qin, G.K. Plattner, M. Tignor, S.K. Allen, J. Boschung, A. Nauels, Y. Xia, V. Bex and P.M. Midgley (eds.)]. Cambridge University Press, Cambridge, United Kingdom and New York, NY, USA, 1137-1216.

Ciais, P. et al., 2013: Carbon and Other Biogeochemical Cycles. In: Climate Change 2013: The Physical Science Basis. Contribution of Working Group I to the Fifth Assessment Report of the Intergovernmental Panel on Climate Change [Stocker, T.F., D. Qin, G.K. Plattner, M. Tignor, S.K. Allen, J. Boschung, A. Nauels, Y. Xia, V. Bex and P.M. Midgley (eds.)]. Cambridge University Press, Cambridge, United Kingdom and New York, NY, USA.

Ciais, P. et al., 2011: Large inert carbon pool in the terrestrial biosphere during the Last Glacial Maximum. Nat. Geosci., 5, 74-79, doi:10.1038/ngeo1324.

Colenbrander, D., A. Cartwright and A. Taylor, 2015: Drawing a line in the sand: Managing coastal risks in the City of Cape Town. S. Afr. Geogr. J., 97(1), 1-17, doi:10.1080/03736245.2014.924865.

Collins, M. et al., 2013: Long-term Climate Change: Projections, Commitments and Irreversibility. In: Climate Change 2013: The Physical Science Basis. Contribution of Working Group I to the Fifth Assessment Report of the Intergovernmental Panel on Climate Change [Stocker, T.F., D. Qin, G.-K. Plattner, M. Tignor, S.K. Allen, J. Boschung, A. Nauels, Y. Xia, V. Bex and P.M. Midgley (eds.)]. Cambridge University Press, Cambridge, United Kingdom and New York, NY, USA, 1029-1136.

Comyn-Platt, E. et al., 2018: Carbon budgets for 1.5 and $2^{\circ} \mathrm{C}$ targets lowered by natural wetland and permafrost feedbacks. Nat. Geosci., 11(8), 568573, doi:10.1038/s41561-018-0174-9.

Corner, A., E. Markowitz and N. Pidgeon, 2014: Public engagement with climate change: The role of human values. WIREs Clim. Change, 5(3), 411422, doi:10.1002/wcc.269.

Cosens, B.A., L. Gunderson and B.C. Chaffin, 2018: Introduction to the Special Feature Practicing Panarchy:Assessing legal flexibility, ecological resilience, and adaptive governance in regional water systems experiencing rapid environmental change. Ecol. Soc., 23(1), 4, doi:10.5751/ES-09524-230104.

Cramer, W. et al., 2014: Detection and attribution of observed impacts. In: Climate Change 2014: Impacts, Adaptation, and Vulnerability. Part A: Global and Sectoral Aspects. Contribution of Working Group II to the Fifth
Assessment Report of the Intergovernmental Panel on Climate Change [Field, C.B., V.R. Barros, D.J. Dokken, K.J. Mach, M.D. Mastrandrea, T.E. Bilir, M. Chatterjee, K.L. Ebi, Y.O. Estrada, R.C. Genova, B. Girma, E.S. Kissel, A.N. Levy, S. MacCracken, P.R. Mastrandrea and L.L. White (eds.)]. Cambridge University Press, Cambridge, United Kingdom and New York, NY, USA, 979-1037.

Crate, S. et al., 2017: Permafrost livelihoods: A transdisciplinary review and analysis of thermokarst-based systems of Indigenous land use. Anthropocene, 18, 89-104, doi:10.1016/j.ancene.2017.06.001.

Crate, S.A. and A.N. Fedorov, 2013: A methodological model for exchanging local and scientific climate change knowledge in northeastern Siberia. Arctic, 66(3), 338-350, doi:10.14430/arctic4312.

Creutzig, F. et al., 2018: Towards demand-side solutions for mitigating climate change. Nat. Clim. Change, 8(4), 260-263, doi:10.1038/s41558-018-0121-1.

Cunsolo, A. and N.R. Ellis, 2018: Ecological grief as a mental health response to climate change-related loss. Nat. Clim. Change, 8(4), 275-281, doi:10.1038/s41558-018-0092-2.

Cunsolo, A. and K. Landman, 2017: Mourning Nature: Hope at the Heart of Ecological Loss and Grief. McGill-Queen's University Press, Montreal-Kingston, $360 \mathrm{pp}$.

Cunsolo Willox, A. et al., 2013: The land enriches the soul: On climatic and environmental change, affect, and emotional health and well-being in Rigolet, Nunatsiavut, Canada. Emote. Space Soc., 6, 14-24, doi:10.1016/j. emospa.2011.08.005.

Czerniewicz, L., S. Goodier and R. Morrell, 2017: Southern knowledge online? Climate change research discoverability and communication practices. Inf. Commun. Soc., 20(3), 386-405, doi:10.1080/1369118X.2016.1168473.

David-Chavez, D.M. and M.C. Gavin, 2018: A global assessment of Indigenous community engagement in climate research. Environ. Res. Letters, 13(12), 123005, doi:10.1088/1748-9326/aaf300.

De Lucia, V., 2017: The Arctic environment and the BBNJ negotiations. Special rules for special circumstances? Mar. Policy, 86, 234-240, doi:10.1016/j. marpol.2017.09.011.

Delworth, T.L. et al., 2017: The central role of ocean dynamics in connecting the North Atlantic Oscillation to the extratropical component of the Atlantic Multidecadal Oscillation. J. Clim., 30(10), 3789-3805, doi:10.1175/ JCLI-D-16-0358.1.

Denton, F. et al., 2014: Climate-resilient pathways: adaptation, mitigation, and sustainable development. In: Climate Change 2014: Impacts, Adaptation, and Vulnerability. Part A: Global and Sectoral Aspects. Contribution of Working Group II to the Fifth Assessment Report of the Intergovernmental Panel on Climate Change [Field, C.B., V. Barros, D.J. Dokken, K.J. Mach, M.D. Mastrandrea, T.E. Bilir, M. Chatterjee, K.L. Ebi, Y.O. Estrada, R.C. Genova, B. Girma, E.S. Kissel, A.N. Levy, S. MacCracken, P.R. Mastrandrea and L.L. White (eds.)]. Cambridge University Press, Cambridge, United Kingdom and New York, NY, USA, 1101-1131.

Deser, C., A. Phillips, V. Bourdette and H. Teng, 2012: Uncertainty in climate change projections: The role of internal variability. Clim. Dyn., 38(3-4), 527-546, doi:10.1007/s00382-010-0977-x.

Dessai, S., M. Hulme, R. Lempert and R.A. Pielke Jr, 2009: Climate prediction: A limit to adaptation? In: Adapting to Climate Change: Thresholds, Values, Governance [Adger, W.N., I. Lorenzoni and K. O'Brien (eds.)]. Cambridge University Press, pp. 64-78. ISBN: 9780521764858.

Deutsch, C. et al., 2015: Climate change tightens a metabolic constraint on marine habitats. Science, 348(6239), 1132-1135, doi:10.1126/science. aaa1605.

DeWalt, B., 1994: Using indigenous knowledge to improve agriculture and natural resource management. Hum. Organ., 53(2), 123-131, doi:10.17730/humo.53.2.ku60563817m03n73.

Díaz, S. et al., 2015: A Rosetta Stone for nature's benefits to people. PLoS Biol., 13(1), e1002040, doi:10.1371/journal.pbio.1002040.

Díaz, S. et al., 2018: Assessing nature's contributions to people. Science, 359(6373), 270-272, doi:10.1126/science.aap8826. 
Djalante, R. et al., 2018: Cross Chapter Box 12: Residual risks, limits to adaptation and loss and damage. In: Global Warming of $1.5^{\circ} \mathrm{C}$. An IPCC Special Report on the impacts of global warming of $1.5^{\circ} \mathrm{C}$ above pre-industrial levels and related global greenhouse gas emission pathways, in the context of strengthening the global response to the threat of climate change, sustainable development, and efforts to eradicate poverty [Masson-Delmotte, V., P. Zhai, H.O. Pörtner, D. Roberts, J. Skea, P.R. Shukla, A. Pirani, W. Moufouma-Okia, C. Péan, R. Pidcock, S. Connors, J.B.R. Matthews, Y. Chen, X. Zhou, M.I. Gomis, E. Lonnoy, T. Maycock, M. Tignor and T. Waterfield (eds.)]. World Meteorological Organization, Geneva, Switzerland.

Dodman, D. and M. Mitlin, 2013: Challenges for community-based adaptation: Discovering the potential for transformation. J. Int. Dev., 25(5), 640-659, doi:10.1002/jid.1772.

Douglas, M. and A. Wildavsky, 1983: Risk and Culture. J. Policy Anal. Manage., 2(2), 221, doi:10.2307/3323308.

Dow, K. et al., 2013: Limits to adaptation. Nat. Clim. Change, 3(4), 305-307, doi:10.1038/nclimate1847.

Dowell, M. et al., 2013: Strategy towards an architecture for climate monitoring from space. Committee on Earth Observation Satellites, pp 39. [Available at: www.wmo.int/pages/prog/sat/.../ARCH_strategy-climat e-architecture-space.pdf].

Downie, C. and M. Williams, 2018: After the Paris Agreement: What Role for the BRICS in Global Climate Governance? Glob. Policy, 9(3), 398-407, doi:10.1111/1758-5899.12550.

Durack, P.J., T. Lee, N.T. Vinogradova and D. Stammer, 2016: Keeping the lights on for global ocean salinity observation. Nat. Clim. Change, 6, 228-231, doi:10.1038/nclimate2946.

Easterling, D.R. et al., 2000: Climate extremes: Observations, modeling, and impacts. Science, 289(5487), 2068-2074, doi:10.1126/ science.289.5487.2068.

Eisenack, K. et al., 2014: Explaining and overcoming barriers to climate change adaptation. Nat. Clim. Change, 4(10), 867-872, doi:10.1038/ nclimate2350.

Ellsberg, D., 1961: Risk, ambiguity, and the Savage axioms. Q.J. Econ., 75(4), 643-669, doi:10.2307/1884324.

Eriksen, S.H., A.J. Nightingale and H. Eakin, 2015: Reframing adaptation: The political nature of climate change adaptation. Global Environ. Change, 35, 523-533, doi:10.1016/j.gloenvcha.2015.09.014.

Escobar, A., 2001: Culture sits in places: Reflections on globalism and subaltern strategies of localization. Political Geogr, 20, 139-174, doi:10.1016/ S0962-6298(00)00064-0.

Eslake, S., 2016: Tasmania Report 2016. Tasmania Chamber of Commerce and Industry, Hobart, Tasmania, 92 [Available at: www.tcci.com.au/ getattachment/Events/Tasmania-Report-2016/Tasmania-Report-2016FINAL.pdf.aspx].

Evans, L.S. et al., 2016: Structural and psycho-social limits to climate change adaptation in the Great Barrier Reef Region. PloS ONE, 11(3), e0150575, doi:10.1371/journal.pone.0150575.

FAO, 2018: The State of World Fisheries and Aquaculture 2018 - Meeting the sustainable development goals. The State of World Fisheries and Aquaculture, FAO, Rome, Italy, 210 pp.

Fazey, I. et al., 2016: Past and future adaptation pathways. Clim. Dev., 8(1), 26-44, doi:10.1080/17565529.2014.989192.

Feldman, L. and P. Hart, 2018: Is there any hope? How climate change news imagery and text influence audience emotions and support for climate mitigation policies. Risk Anal., 38(3), 585-602, doi:10.1111/risa.12868.

Feldman, L. and P.S. Hart, 2016: Using political efficacy messages to increase climate activism: The mediating role of emotions. Sci. Commun., 38(1), 99-127, doi:10.1177/1075547015617941.

Fenichel, E.P. et al., 2016: Wealth reallocation and sustainability under climate change. Nat. Clim. Change, 6(3), 237-244, doi:10.1038/nclimate2871.
Fenty, I., D. Menemenlis and H. Zhang, 2017: Global coupled sea ice-ocean state estimation. Clim. Dyn., 49(3), 931-956, doi:10.1007/s00382-015-2796-6.

Few, R. et al., 2017: Transformation, adaptation and development: relating concepts to practice. Palgrave Commun., 3, doi:10.1057/ palcomms.2017.92.

Field, C.B. et al., 2014: Technical summary. In: Climate Change 2014: Impacts, Adaptation, and Vulnerability. Part A: Global and Sectoral Aspects. Contribution of Working Group II to the Fifth Assessment Report of the Intergovernmental Panel on Climate Change [Field, C.B., V.R. Barros, D.J. Dokken, K.J. Mach, M.D. Mastrandrea, T.E. Biller, M. Chatterjee, K.L. Ebi, Y.O. Estrada, R.C. Genova, B. Girma, E.S. Kissel, A.N. Levy, S. MacCracken, P.R. Mastrandrea and L.L. White (eds.)]. Cambridge University Press, Cambridge, United Kingdom and New York, NY, USA, 35-94.

Field, C.B., M.J. Behrenfeld, J.T. Randerson and P. Falkowski, 1998: Primary production of the biosphere: Integrating terrestrial and oceanic components. Science, 281(5374), 237-240, doi:10.1126/science.281.5374.237.

Fischer, H. et al., 2018: Palaeoclimate constraints on the impact of $2^{\circ} \mathrm{C}$ anthropogenic warming and beyond. Nat. Geosci., 11, 474-485, doi:10.1038/s41561-018-0146-0.

Fischer, J. et al., 2015: Advancing sustainability through mainstreaming a social-ecological systems perspective. Curr. Opin. Environ. Sustain., 14, 144-149, doi:10.1016/j.cosust.2015.06.002.

Flato, G. et al., 2013: Evaluation of climate models In: Climate Change 2013: The Physical Science Basis. Contribution of Working Group I to the Fifth Assessment Report of the Intergovernmental Panel on Climate Change [Stocker, T.F., D. Qin, G.-K. Plattner, M. Tignor, S.K. Allen, J. Boschung, A. Nauels, Y. Xia, V. Bex and P.M. Midgley (eds.)]. Cambridge University Press, Cambridge, United Kingdom and New York, NY, USA, 741-866.

Florano, E.R., 2018: Integrated Loss and Damage-Climate Change Adaptation-Disaster Risk Reduction Framework: The Case of the Philippines. In: Resilience [Zommers, Z. (ed.)]. Elsevier, Amsterdam, The Netherlands, pp. 317-324.

Flynn, M. et al., 2018: Participatory scenario planning and climate change impacts, adaptation and vulnerability research in the Arctic. Environmental Science \& Policy, 79, 45-53, doi:10.1016/j.envsci.2017.10.012.

Ford, J.D. et al., 2016a: Including indigenous knowledge and experience in IPCC assessment reports. Nat. Clim. Change, 6 (4), 349-353, doi:10.1038/ nclimate2954.

Ford, J.D. et al., 2019: Changing access to ice, land and water in Arctic communities. Nat. Clim. Change, 9, 335-339, doi:10.1038/s41558-0190435-7.

Ford, J.D., N. Couture, T. Bell and D.G. Clark, 2017: Climate change and Canada's north coast: Research trends, progress, and future directions. Environmental Reviews, 26(1), 82-92, doi:10.1139/er-2017-0027.

Ford, J.D. et al., 2016b: Adaptation and indigenous peoples in the United Nations framework convention on climate change. Clim. Change, 139(34), 429-443.

Forino, G., J. von Meding and G.J. Brewer, 2015: A conceptual governance framework for climate change adaptation and disaster risk reduction integration. Int. J. Disaster Risk Sc., 6(4), 372-384, doi:10.1007/s13753015-0076-z.

Frame, B. et al., 2018: Adapting global shared socio-economic pathways for national and local scenarios. Clim. Risk Manage., 21, 39-51, doi:10.1016/j. crm.2018.05.001.

Fretwell, P. et al., 2013: Bedmap2: Improved ice bed, surface and thickness datasets for Antarctica. The Cryosphere, 7, 375-393, doi:10.5194/tc-7375-2013.

Frölicher, T.L. and F. Joos, 2010: Reversible and irreversible impacts of greenhouse gas emissions in multi-century projections with the NCAR global coupled carbon cycle-climate model. Clim. Dyn., 35(7-8), 14391459, doi:10.1007/s00382-009-0727-0.

Frölicher, T.L., K.B. Rodgers, C.A. Stock and W. Cheung, 2016: Sources of uncertainties in 21 st century projections of potential ocean 
ecosystem stressors. Global Biogeochem. Cycles, 30(8), 1224-1243, doi:10.1002/2015GB005338.

Gaire, K., R. Beilin and F. Miller, 2015: Withdrawing, resisting, maintaining and adapting: Food security and vulnerability in Jumla, Nepal. Reg. Environ. Change, 15(8), 1667-1678, doi:10.1007/s10113-014-0724-7.

Gallina, V. et al., 2016:A review of multi-risk methodologies for natural hazards: Consequences and challenges for a climate change impact assessment. J. Environ. Manage., 168, 123-132, doi:10.1016/j.jenvman.2015.11.011.

Gallo, N.D., D.G. Victor and L.A. Levin, 2017: Ocean commitments under the Paris Agreement. Nat. Clim. Change, 7, 833-838, doi:10.1038/ nclimate3422.

Gasser, T. et al., 2015: Negative emissions physically needed to keep global warming below $2^{\circ} \mathrm{C}$. Nat. Commun., 6, 7958, doi:10.1038/ncomms8958.

Gattuso, J.-P. et al., 2014: Ocean Acidification. In: Climate Change 2014: Impacts, Adaptation, and Vulnerability. Part A: Global and Sectoral Aspects. Contribution of Working Group II to the Fifth Assessment Report of the Intergovernmental Panel on Climate Change [Field, C.B., V.R. Barros, D.J. Dokken, K.J. Mach, M.D. Mastrandrea, T.E. Biller, M. Chatterjee, K.L. Ebi, Y.O. Estrada, R.C. Genova, B. Girma, E.S. Kissel, A.N. Levy, S. MacCracken, P.R. Mastrandrea and L.L. White (eds.)]. Cambridge University Press, Cambridge, United Kingdom and New York, NY, USA, 129-131.

Gattuso, J.-P. et al., 2015: Contrasting futures for ocean and society from different anthropogenic $\mathrm{CO}_{2}$ emissions scenarios. Science, 349(6243), 45-55, doi:10.1126/science.aac4722.

Gattuso, J.-P. et al., 2018: Ocean solutions to address climate change and its effects on marine ecosystems. Front. Mar. Sci., 5(337), doi:10.3389/ fmars.2018.00337.

Gearheard, S.F. et al., 2013: The Meaning of Ice: People and Sea Ice in Three Arctic Communities. International Polar Institute Press Hanover, New Hampshire, 366 pp. ISBN: 9780996193856.

Gebbie, G. and P. Huybers, 2012: The mean age of ocean waters inferred from radiocarbon observations: Sensitivity to surface sources and accounting for mixing histories. J. Phys. Oceanogr., 42(2), 291-305, doi:10.1175/ JPO-D-11-043.1.

Gerrard, M.B. and G.E. Wannier, 2013: Threatened Island Nations: Legal implications of rising seas and a changing climate. Cambridge University Press, Cambridge, 627 pp. ISBN: 978113919877.

Ghalambor, C.K. et al., 2015: Non-adaptive plasticity potentiates rapid adaptive evolution of gene expression in nature. Nature, 525(7569), 372375, doi:10.1038/nature15256.

Gimeno, L. et al., 2012: Oceanic and terrestrial sources of continental precipitation. Rev. Geophys, 50(4), RG4003, doi:10.1029/2012RG000389.

Gleick, P.H., 1996: Water resources. In: Encyclopedia of Climate and Weather [Schneider, S.H. (ed.)]. Oxford University Press, New York, pp. 817-823. ISBN 978-0816063505.

Glibert, P.M. et al., 2014: Vulnerability of coastal ecosystems to changes in harmful algal bloom distribution in response to climate change: Projections based on model analysis. Glob. Chang. Biol., 20(12), 38453858, doi:10.1111/gcb.12662.

Gobler, C.J. et al., 2017: Ocean warming since 1982 has expanded the niche of toxic algal blooms in the North Atlantic and North Pacific oceans. Proc. Natl. Acad. Sci. U.S.A., 114(19), 4975-4980, doi:10.1073/pnas.1619575114.

Goldman, M.J. and E. Lovell, 2017: Indigenous Technical Knowledge. In: International Encyclopedia of Geography: People, the Earth, Environment and Technology [Richardson, D., N. Castree, M.F. Goodchild, A. Kobayashi, W. Liu and R.A. Marston (eds.)]. Wiley, pp. 1-4. ISBN 9781118786352.

Golledge, N.R. et al., 2019: Global environmental consequences of twenty-first-century ice-sheet melt. Nature, 566, 65-72, doi:10.1038/ s41586-019-0889-9.

Golledge, N.R. et al., 2015: The multi-millennial Antarctic commitment to future sea-level rise. Nature, 526(7573), 421-425, doi:10.1038/ nature15706.
Graham, J., T.W. Plumptre and B. Amos, 2003: Principles for Good Governance in the 21st Century. IOG Policy Briefs, Institute on Governance Ottawa, 9 pp.

Green, D., J. Billy and A. Tapim, 2010: Indigenous Australians' knowledge of weather and climate. Clim. Change, 100(2), 337-354, doi:10.1007/ s10584-010-9803-z.

Grip, K., 2017: International marine environmental governance: A review. Ambio, 46(4), 413-427, doi:10.1007/s13280-016-0847-9.

Grosse, G. et al., 2016: Changing permafrost in a warming world and feedbacks to the Earth system. Environ. Res. Letters, 11(4), 04021, doi:10.1088/17489326/11/4/040201.

Gruber, N. et al., 2019: The oceanic sink for anthropogenic $\mathrm{CO}_{2}$ from 1994 to 2007. Science, 363(6432), 1193-1199, doi:10.1126/science.aau5153.

Grunblatt, J. and L. Alessa, 2017: Role of perception in determining adaptive capacity: communities adapting to environmental change. Sustain. Sci., 12(1), 3-13, doi:10.1007/s11625-016-0394-0.

Gupta, A.S., N.C. Jourdain, J.N. Brown and D. Monselesan, 2013: Climate drift in the CMIP5 models. J. Clim., 26(21), 8597-8615, doi:10.1175/ JCLI_D_12_00521.1.

Haasnoot, M., J.H. Kwakkel, W.E. Walker and J. ter Maat, 2013: Dynamic adaptive policy pathways: A method for crafting robust decisions for a deeply uncertain world. Global Environ. Change, 23(2), 485-498, doi:10.1016/j.gloenvcha.2012.12.006.

Hall, N. and $\AA$. Persson, 2018: Global climate adaptation governance: Why is it not legally binding? Eur. J. Int. Relat., 24(3), 540-566, doi:10.1177/1354066117725157.

Halpern, B.S. et al., 2015: Spatial and temporal changes in cumulative human impacts on the world's ocean. Nat. Commun., 6, 7615, doi:10.1038/ ncomms8615.

Halpern, B.S. et al., 2008: A global map of human impact on marine ecosystems. Science, 319(5865), 948-952, doi:10.1126/science.1149345.

Hamilton, M.L. and M. Lubell, 2019: Climate change adaptation, social capital, and the performance of polycentric governance institutions. Clim. Change, 152(3-4), 307-326, doi:10.1007/s10584-019-02380-2.

Hansell, D.A., 2013: Recalcitrant dissolved organic carbon fractions. Annu. Rev. Mar. Sci, 5(1), 421-445, doi:10.1146/annurev-marine-120710-100757.

Hansen, J. et al., 2016: Ice melt, sea level rise and superstorms: Evidence from paleoclimate data, climate modeling, and modern observations that $2^{\circ} \mathrm{C}$ global warming could be dangerous. Atmos. Chem. Phys., 16(6), 37613812, doi:10.5194/acp-16-3761-2016.

Hansen, J. et al., 2007: Climate change and trace gases. Philosophical Transactions of the Royal Society A: Mathematical, Physical and Engineering Sciences, 365(1856), 1925-1954, doi:10.1098/rsta.2007.2052.

Hansen, J., M. Sato, P. Kharecha and K. von Schuckmann, 2011: Earth's energy imbalance and implications. Atmos. Chem. Phys., 11(24), 13421-13449, doi:10.5194/acp-11-13421-2011.

Hansson, S.O. and G. Hirsch Hadorn, 2017: Argument-based decision support for risk analysis. J. Risk Res., 21(12), 1449-1464, doi:10.1080/13669877. 2017.1313767.

Hare, J.A. et al., 2016: A vulnerability assessment of fish and invertebrates to climate change on the Northeast US Continental Shelf. PloS ONE, 11(2), e0146756, doi:10.1371/journal.pone.0146756.

Hastrup, K., A. Mosbech and B. Grønnow, 2018: Introducing the North Water: Histories of exploration, ice dynamics, living resources, and human settlement in the Thule Region. Ambio, 47(2), 162-174, doi:10.1007/ s13280-018-1030-2.

Hawkins, E. et al., 2017: Estimating changes in global temperature since the preindustrial period. Bull. Am. Meteorol. Soc., 98(9), 1841-1856, doi:10.1175/BAMS-D-16-0007.1.

Hawkins, E. and R. Sutton, 2009: The potential to narrow uncertainty in regional climate predictions. Bull. Am. Meteorol. Soc., 90(8), 1095-1108, doi:10.1175/2009BAMS2607.1.

Hawkins, E. and R. Sutton, 2012: Time of emergence of climate signals. Geophys. Res. Letters, 39(1), L01702, doi:10.1029/2011GL050087. 
Hegerl, G.C. et al., 2010: Good practice guidance paper on detection and attribution related to anthropogenic climate change. In: Meeting Report of the Intergovernmental Panel on Climate Change Expert Meeting on Detection and Attribution of Anthropogenic Climate Change [Stocker, T.F., C.B. Field, D. Qin, V. Barros, G.-K. Plattner, M. Tignor, P.M. Midgley and K.L. Ebi (eds.)]. IPCC Working Group I Technical Support Unit, University of Bern, Bern, Switzerland.

Heleniak, T., 2014: Arctic Populations and Migration. Arctic Human Development Report: Regional Processes and Global Linkages, Nordic Council of Ministers, Copenhagen. 53-104 pp.

Hijioka, Y. et al., 2014: Asia. In: Climate Change 2014: Impacts, Adaptation, and Vulnerability. Part B: Regional Aspects: Working Group II Contribution to the Fifth Assessment Report of the Intergovernmental Panel on Climate Change [Barros, V.R., C.B. Field, D.J. Dokken, M.D. Mastrandrea, K.J. Mach, T.E. Bilir, M. Chatterjee, K.L. Ebi, Y.O. Estrada, R.C. Genova, B. Girma, E.S. Kissel, A.N. Levy, S. MacCracken, P.R. Mastrandrea and L.L. White (eds.)]. Cambridge University Press, Cambridge, United Kingdom and New York, NY, USA, 1327-1370.

Hinkel, J. et al., 2014: Coastal flood damage and adaptation costs under 21st century sea-level rise. Proc. Natl. Acad. Sci. U.S.A., 111(9), 3292-3297, doi:10.1073/pnas.1222469111.

Hinners, J., A. Kremp and I. Hense, 2017: Evolution in temperature-dependent phytoplankton traits revealed from a sediment archive: Do reaction norms tell the whole story? Proc. R. Soc. Lond. B., 284(1864), 20171888, doi:10.1098/rspb.2017.1888.

Hino, M., C.B. Field and K.J. Mach, 2017: Managed retreat as a response to natural hazard risk. Nat. Clim. Change, 7(5), 364-370, doi:10.1038/ nclimate3252.

Hiwasaki, L., E. Luna and S.R. Syamsidik, 2014: Local and indigenous knowledge for community resilience: Hydro-meteorological disaster risk reduction and climate change adaptation in coastal and small island communities. UNESCO, Jakarta, 60 pp. ISBN: 9786029416114.

Hoegh-Guldberg, 0 . et al., 2018: Impacts of $1.5^{\circ} \mathrm{C}$ global warming on natural and human systems. In: Global Warming of $1.5^{\circ} \mathrm{C}$. An IPCC Special Report on the impacts of global warming of $1.5^{\circ} \mathrm{C}$ above pre-industrial levels and related global greenhouse gas emission pathways, in the context of strengthening the global response to the threat of climate change, sustainable development, and efforts to eradicate poverty [Masson-Delmotte, V., P. Zhai, H.O. Pörtner, D. Roberts, J. Skea, P.R. Shukla, A. Pirani, W. Moufouma-Okia, C. Péan, R. Pidcock, S. Connors, J.B.R. Matthews, Y. Chen, X. Zhou, M.I. Gomis, E. Lonnoy, T. Maycock, M. Tignor and T. Waterfield (eds.)]. World Meteorological Organization, Geneva, Switzerland.

Homsy, G.C., Z. Liu and M.E. Warner, 2019: Multilevel governance: Framing the integration of top-down and bottom-up policymaking. Int. J. Public Adm., 42(7), 572-582.

Horton, B.P., S. Rahmstorf, S.E. Engelhart and A.C. Kemp, 2014: Expert assessment of sea-level rise by AD 2100 and AD 2300. Quaternary Sci. Rev., 84, 1-6, doi:10.1016/j.quascirev.2013.11.002.

Hou, L., J. Huang and J. Wang, 2017: Early warning information, farmers' perceptions of, and adaptations to drought in China. Clim. Change, 141(2), 197-212, doi:10.1007/s10584-017-1900-9.

Hovelsrud, G.K., B. Poppel, B. Van Oort and J.D. Reist, 2011: Arctic societies, cultures, and peoples in a changing cryosphere. Ambio, 40, 100-110, doi:10.1007/s13280-011-0219-4.

$\mathrm{Hu}$, S. and J. Sprintall, 2016: Interannual variability of the Indonesian Throughflow: The salinity effect. J. Geophys. Res. Oceans, 121(4), 25962615, doi:10.1002/2015JC011495.

Huggel, C. et al., 2019: Loss and damage in the mountain cryosphere. Reg. Environ. Change, 19(5), 1387-1399, doi:10.1007/s10113-018-1385-8.

Hughes, T.P. et al., 2018: Spatial and temporal patterns of mass bleaching of corals in the Anthropocene. Science, 359(6371), 80-83, doi:10.1126/ science.aan8048.
Huntington, H.P., 1992: The Alaska Eskimo Whaling Commission and other cooperative marine mammal management organizations in northern Alaska. Polar Rec., 28(165), 119-126, doi:10.1017/S0032247400013413.

Huntington, H.P., 2000: Using traditional ecological knowledge in science: methods and applications. Ecological Applications, 10(5), 1270-1274, doi:10.1890/1051-0761(2000)010[1270:UTEKIS]2.0.C0;2.

Huntington, H.P., 2011: Arctic science: The local perspective. Nature, 478(7368), 182-183, doi:10.1038/478182a.

Huntington, H.P. et al., 2017: How small communities respond to environmental change: Patterns from tropical to polar ecosystems. Ecol. Soc., 22(3), 9.

Huq, S., E. Roberts and A. Fenton, 2013: Loss and damage. Nat. Clim. Change, 3, 947-949, doi:10.1038/nclimate2026.

Huybrechts, P. and J. de Wolde, 1999: The dynamic response of the Greenland and Antarctic ice sheets to multiple-century climatic warming. J. Clim., 12 (8), 2169-2188, doi:10.1175/1520-0442(1999)012<2169:TDROTG>2.0.C0;2.

ICC, 2015: Inuit Circumpolar Council, Alaskan Inuit Food Security Conceptual Framework: How to assess the Arctic from an Inuit perspective. Alaska [Available at: https://iccalaska.org/wp-icc/wp-content/uploads/2016/05/ Food-Security-Full-Technical-Report.pdf].

ICC. 2018: People of the Ice Bridge: The Future of the Pikialasorsuaq. [Available at: http://pikialasorsuaq.org/en/, accessed 23 March].

ICC, 2018: Utqiagivik Declaration. Inuit Circumpolar Council, Utqiagvik, Alaska [Available at: www.inuitcircumpolar.com/uploads/3/0/5/4/30542 564/2018_utqiagvik_declaration_-_final.pdf].

Inniss, L. and A. Simcock, 2017: The First Global Integrated Marine Assessment: World Ocean Assessment I. World Ocean Assessment, Cambridge University Press, Cambridge, United Kingdom, 973 pp. ISBN: 9781108186148.

IPBES, 2018a: The IPBES regional assessment report on biodiversity and ecosystem services for Africa.[Archer, E., L. Dziba, K.J. Mulongoy, M.A. Maoela and M. Walters (eds.)]. Secretariat of the Intergovernmental Science-Policy Platform on Biodiversity and Ecosystem services, Bonn, Germany, 492 pp. ISBN: 9783947851003.

IPBES, 2018b: The IPBES regional assessment report on biodiversity and ecosystem services for Asia and the Pacific.[Senaratna Sellamuttu, S., M. Karki, N. Moriwake and S. Okayasu (eds.)] [Karki, M., S. Senaratna Sellamuttu, S. Okayasu and W. Suzuki (eds.)]. Secretariat of the Intergovernmental Science-Policy Platform on Biodiversity and Ecosystem services, Bonn, Germany, 612 pp. ISBN: 9783947851027.

IPBES, 2018c: The IPBES regional assessment report on biodiversity and ecosystem services for Europe and Central Asia.[Rounsevell, M., M. Fischer, A. Torre-Marin Rando and A. Mader (eds.)]. Secretariat of the Intergovernmental Science-Policy Platform on Biodiversity and Ecosystem services, Bonn, Germany, 892 pp. ISBN: 9783947851034.

IPBES, 2018d: The IPBES regional assessment report on biodiversity and ecosystem services for the Americas. [Rice, J., C.S. Seixas, M.E. Zaccagnini, M. Bedoya-Gaitán and N. Valderrama (eds.)]. Secretariat of the Intergovernmental Science-Policy Platform on Biodiversity and Ecosystem Services, Bonn, Germany, 656 pp. ISBN: 9783947851010.

IPCC, 2000: Emissions Scenarios [Nakicenovic, N. and R. Swart (eds.)]. Cambridge University Press, Cambridge, United Kingdom, 570 pp.

IPCC, 2013: Summary for Policymakers. In: Climate Change 2013: The Physical Science Basis. Contribution of Working Group I to the Fifth Assessment Report of the Intergovernmental Panel on Climate Change [Stocker, T.F., D. Qin, G.-K. Platter, M. Tignor, S.K. Allen, J. Boschung, A. Nauels, Y. Xia, V. Bex and P.M. Midgley (eds.)]. Cambridge University Press, Cambridge, United Kingdom and New York, NY, USA.

IPCC, 2014: Summary for Policymakers. In: Climate Change 2014: Impacts, Adaptation, and Vulnerability. Part A: Global and Sectoral Aspects. Contribution of Working Group II to the Fifth Assessment Report of the Intergovernmental Panel on Climate Change [Field, C.B., V. Barros, D.J. Dokken, K.J. Mach, M.D. Mastrandrea, T.E. Bilir, M. Chatterjee, K.L. Ebi, Y.O. Estrada, R.C. Genova, B. Girma, E.S. Kissel, A.N. Levy, S. MacCracken, 
M.P.R. and L.L. White (eds.)]. Cambridge University Press, Cambridge, United Kingdom and New York, NY, USA, 1-32.

IPCC, 2018: Summary for Policymakers. In: Global Warming of $1.5^{\circ} \mathrm{C}$. An IPCC Special Report on the impacts of global warming of $1.5^{\circ} \mathrm{C}$ above pre-industrial levels and related global greenhouse gas emission pathways, in the context of strengthening the global response to the threat of climate change, sustainable development, and efforts to eradicate poverty [Masson-Delmotte, V., P. Zhai, H.O. Pörtner, D. Roberts, J. Skea, P.R. Shukla, A. Pirani, W. Moufouma-Okia, C. Péan, R. Pidcock, S. Connors, J.B.R. Matthews, Y. Chen, X. Zhou, M.I. Gomis, E. Lonnoy, T. Maycock, M. Tignor and T. Waterfield (eds.)]. World Meteorological Organization, Geneva, Switzerland, 32 pp.

ITK, 2005: Putting the human face on climate change: Perspectives from Inuit in Canada. Inuit Tapiriit Kanatami, Nasivvik Centre for Inuit Health and Changing Environments at Université Laval and the Ajunnginiq Centre at the National Aboriginal Health Organization, Unikkaaqatigiit [Available at: www.itk.ca/wp-content/uploads/2016/07/unikkaaqatigiit01-1.pdf.].

Jamir, 0., 2016: Understanding India-Pakistan water politics since the signing of the Indus Water Treaty. Water Policy, 18(5), 1070-1087, doi:https://doi. org/10.2166/wp.2016.185.

Jasanoff, S., 2004: States of knowledge: The co-production of science and the social order. Routledge, London and New York, 332 pp. ISBN: 9780415403290.

Jiao, N. et al., 2010: Microbial production of recalcitrant dissolved organic matter: Long-term carbon storage in the global ocean. Nat. Rev. Microbiol., 8(8), 593-599, doi:10.1038/nrmicro2386.

Johnson, G.C. et al., 2018: Ocean heat content. In: State of the Climate 2017 [Blunden, J., D.S. Arndt and G. Hartifled (eds.)]. Bull. Am. Meteorol. Soc, 99(8), S72-S77.

Jones, H.P., D.G. Hole and E.S. Zavaleta, 2012: Harnessing nature to help people adapt to climate change. Nat. Clim. Change, 2(7), 504-509, doi:10.1038/nclimate1463.

Jones, J.M. et al., 2016: Assessing recent trends in high-latitude Southern Hemisphere surface climate. Nat. Clim. Change, 6(10), 917-926, doi:10.1038/nclimate3103.

Jordan, A.J. et al., 2015: Emergence of polycentric climate governance and its future prospects. Nat. Clim. Change, 5(11), 977-982, doi:10.1038/ nclimate2725.

Jupiter, S.D. et al., 2014: Locally-managed marine areas: Multiple objectives and diverse strategies. Pac. Conserv. Biol., 20(2), 165-179, doi:10.1071/ PC140165.

Kahneman, D. and P. Egan, 2011: Thinking, fast and slow. Farrar, Straus and Giroux, New York, 499 pp. ISBN: 9780141033570.

Kainuma, M. et al., 2018: Cross-Chapter Box 1: Scenarios and Pathways. In: Global Warming of $1.5^{\circ} \mathrm{C}$. An IPCC Special Report on the impacts of global warming of $1.5^{\circ} \mathrm{C}$ above pre-industrial levels and related global greenhouse gas emission pathways, in the context of strengthening the global response to the threat of climate change, sustainable development, and efforts to eradicate poverty. [Masson-Delmotte, V., P. Zhai, H.O. Pörtner, D. Roberts, J. Skea, P.R. Shukla, A. Pirani, W. Moufouma-Okia, C. Péan, R. Pidcock, S. Connors, J.B.R. Matthews, Y. Chen, X. Zhou, M.I. Gomis, E. Lonnoy, T. Maycock, M. Tignor and T. Waterfield (eds.)]. World Meteorological Organization, Geneva, Switzerland, 62-64.

Kanna, N. et al., 2018: Upwelling of macronutrients and dissolved inorganic carbon by a subglacial freshwater driven plume in Bowdoin Fjord, northwestern Greenland. J. Geophys. Res. Biogeosci., 123(5), 1666-1682, doi:10.1029/2017JG004248.

Karim, M.S., 2015: Prevention of pollution of the marine environment from vessels: The potential and limits of the International Maritime Organisation. Springer. ISBN: 9783319106083.

Karki, M. et al., 2017: Knowing our lands and resources: Indigenous and local knowledge and practices related to biodiversity and ecosystem services in Asia. Knowledges of Nature 10, UNESCO, Paris, 212 pp. ISBN: 9789231002106.

Karlsson, M. and G.K. Hovelsrud, 2015: Local collective action: Adaptation to coastal erosion in the Monkey River Village, Belize. Global Environ. Change, 32, 96-107, doi:10.1016/j.gloenvcha.2015.03.002.

Kaser, G., M. Großhauser and B. Marzeion, 2010: Contribution potential of glaciers to water availability in different climate regimes. Proc. Natl. Acad. Sci. U.S.A., 107, 20223-20227, doi:10.1073/pnas.1008162107.

Keeling, C.D. et al., 1976: Atmospheric carbon dioxide variations at Mauna Loa observatory, Hawaii. Tellus, 28 (6),538-551, doi:10.1111/j.2153-3490.1976. tb00701.x.

Keller, K.M., F. Joos and C. Raible, 2014: Time of emergence of trends in ocean biogeochemistry. Biogeosciences, 11(13), 3647-3659, doi:10.5194/bg-113647-2014.

Kilroy, G., 2015: A review of the biophysical impacts of climate change in three hotspot regions in Africa and Asia. Reg. Environ. Change, 15 (5), 771-782, doi:10.1007/s10113-014-0709-6.

Kirchmeier-Young, M.C., F.W. Zwiers and N.P. Gillett, 2017: Attribution of extreme events in Arctic sea ice extent. J. Clim., 30 (2), 553-571, doi:10.1175/JCLI-D-16-0412.1.

Klein, R.J.T. et al., 2014: Adaptation opportunities, constraints, and limits. In: Climate Change 2014: Impacts, Adaptation, and Vulnerability. Part A: Global and Sectoral Aspects. Contribution of Working Group II to the Fifth Assessment Report of the Intergovernmental Panel on Climate Change [Field, C.B., V.R. Barros, D.J. Dokken, K.J. Mach, M.D. Mastrandrea, T.E. Bilir, M. Chatterjee, K.L. Ebi, Y.O. Estrada, R.C. Genova, B. Girma, E.S. Kissel, A.N. Levy, S. MacCracken, P.R. Mastrandrea and L.L. White (eds.)]. Cambridge University Press, Cambridge, United Kingdom and New York, NY, USA, 899-943.

Kleisner, K.M. et al., 2017: Marine species distribution shifts on the US Northeast Continental Shelf under continued ocean warming. Prog. Oceanogr., 153, 24-36, doi:10.1016/j.pocean.2017.04.001.

Klenk, N. and K. Meehan, 2015: Climate change and transdisciplinary science: Problematizing the integration imperative. Environmental Science \& Policy, 54, 160-167, doi:10.1016/j.envsci.2015.05.017.

Klinsky, S. et al., 2016: Why equity is fundamental in climate change policy research. Global Environ. Change, 44, 170-173, doi:10.1016/j. gloenvcha.2016.08.002.

Knight, F.H., 1921: Risk, uncertainty and profit. University of Chicago Press, Chicago, 381 pp. ISBN: 9781614276395.

Knoblauch, C. et al., 2018: Methane production as key to the greenhouse gas budget of thawing permafrost. Nat. Clim. Change, 8(4), 309, doi:10.1038/ s41558-018-0095-z.

Knutson, T. et al., 2017: Detection and attribution of climate change. In: Climate Science Special Report: Fourth National Climate Assessment, Volume I.U.S. Global Change Research Program, Washington DC, USA, pp.114-132.

Knutti, R.F., T.F. Stocker and A. Timmermann, 2004: Strong hemispheric coupling of glacial climate through freshwater discharge and ocean circulation. Nature, 430(7002), 851-856, doi:10.1038/nature02786.

Kofinas, G. et al., 2013: Adaptive and transformative capacity. In: Arctic Resilience Interim Report to the Arctic Council. Stockholm Environment Institute and Stockholm Resilience Centre, Stockholm, pp. 71-91. ISBN: 9789186125424.

Koivurova, T., 2016: Arctic resources: Exploitation of natural resources in the arctic from the perspective of international law. In: Research Handbook on International Law and Natural Resources [Morgera, E. and K. Kulovesi (eds.)]. Edward Elgar Publishing, pp. 349-367. ISBN: 9781783478323.

Kokelj, S.V. et al., 2015: Increased precipitation drives mega slump development and destabilization of ice-rich permafrost terrain, northwestern Canada. Glob. Planet. Change, 129, 56-68, doi:10.1016/j.gloplacha.2015.02.008. 
Kopp, R.E., R.L. Shwom, G. Wagner and J. Yuan, 2016: Tipping elements and climate-economic shocks: Pathways toward integrated assessment. Earth's Future, 4(8), 346-372, doi:10.1002/2016EF000362.

Kriegler, E. et al., 2016: Fossil-fueled development (SSP5): an energy and resource intensive scenario for the 21st century. Global Environ. Change, 42, 297-315, doi:10.1016/j.gloenvcha.2016.05.015.

Kulonen, K., Adler, C., Bracher, C., and S. Wymann von Dach, 2019: Spatial context matters for monitoring and reporting on SDGs: Reflections based on research in mountain regions. GAIA, 28(2), 90-94(5), doi.org/10.14512/ gaia.28.2.5.

Kummu, M. et al., 2016: Over the hills and further away from coast: global geospatial patterns of human and environment over the 20th-21st centuries. Environ. Res. Letters, 11(3), 034010, doi:10.1088/17489326/11/3/034010.

Lagerloef, G., R. Schmitt, J. Schanze and H.-Y. Kao, 2010: The ocean and the global water cycle. Oceanography, 23(4), 82-93, doi:10.5670/ oceanog.2010.07.

Lavrillier, A. and S. Gabyshev, 2017: An Arctic indigenous knowledge system of landscape, climate, and human interactions. Evenki Reindeer Herders and Hunters, Studies in Social and Cultural Anthropology, SEC Publications, Kulturstiftung Sibirien, Fürstenberg/Havel, Germany, 467 pp. ISBN: 9783942883313.

Lavrillier, A. and S. Gabyshev, 2018: An emic science of climate: A reindeer evenki environmental knowledge and the notion of an extreme process of change. In: Human-environment relationships in Siberia and Northeast China: Skills, rituals, mobility and politics among the Tungus peoples [Lavrillier, A., A. Dumont and D. Brandisauskas (eds.)]. EMSCAT, 49.

Lawrence, J. et al., 2018: National guidance for adapting to coastal hazards and sea-level rise: Anticipating change, when and how to change pathway. Environmental Science \& Policy, 82, 100-107, doi:10.1016/j. envsci.2018.01.012.

Le Quéré, C. et al., 2018: Global Carbon Budget 2018. Earth Syst. Sci. Data, 10(4), doi:10.5194/essd-10-2141-2018.

LeBlanc, D., C. Freire and M. Vierros, 2017: Mapping the linkages between oceans and other Sustainable Development Goals. DESA Working Paper No. 149, ST/ESA/2017/DWP/149 [Available at: www.un.org/en/development/ desa/pa].

Lee, T.M. et al., 2015: Predictors of public climate change awareness and risk perception around the world. Nat. Clim. Change, 5(11), 1014-1020, doi:10.1038/nclimate2728.

Lele, S., V. Srinivasan, B.K. Thomas and P. Jamwal, 2018: Adapting to climate change in rapidly urbanizing river basins: Insights from a multiple-concerns, multiple-stressors, and multi-level approach. Water Int., 43(2), 281-304, doi:10.1080/02508060.2017.1416442.

Lellouche, J. et al., 2018: Recent updates on the Copernicus Marine Service global ocean monitoring and forecasting real-time 1/12 high resolution system. Ocean Sci., 14(5), 1093-1126, doi:10.5194/os-2018-15.

Lempert, R.J., S.W. Popper and S.C. Bankes, 2003: Shaping the next one hundred years: New methods for quantitative, long-term policy analysis. RAND Corporation, Santa Monica, CA, 186 pp. ISBN: 9780833032751.

Lempert, R.J., S.W. Popper and S.C. Bankes, 2010: Robust Decision Making: Coping with Uncertainty. The Futurist, 44(1), 47-48.

Lenton, T.M. et al., 2008: Tipping elements in the Earth's climate system. Proc. Natl. Acad. Sci. U.S.A., 105(6), 1786-1793, doi:10.1073/pnas.0705414105.

Lin, P.-F. et al., 2016: Long-term surface air temperature trend and the possible impact on historical warming in CMIP5 models. Atmos. Ocean Sci. Letters, 9(3), 153-161, doi:10.1080/16742834.2016.1159911.

Lindgren, A., G. Hugelius and P. Kuhry, 2018: Extensive loss of past permafrost carbon but a net accumulation into present-day soils. Nature, 560 (7717), 219-222, doi:10.1038/s41586-018-0371-0.

Liu, J. et al., 2016: Has Arctic sea ice loss contributed to increased surface melting of the Greenland ice sheet? J. Clim., 29(9), 3373-3386, doi:10.1175/JCLI-D-15-0391.1.
Lüthi, D. et al., 2008: High-resolution carbon dioxide concentration record 650,000-800,000 years before present. Nature, 453, 379-382, doi:10.1038/nature06949.

Mach, K.J., M.D. Mastrandrea, T.E. Bilir and C.B. Field, 2016: Understanding and responding to danger from climate change: The role of key risks in the IPCC AR5. Clim. Change, 136(3-4), 427-444, doi:10.1007/s10584-016-1645-x.

Mach, K.J., M.D. Mastrandrea, P.T. Freeman and C.B. Field, 2017: Unleashing expert judgment in assessment. Global Environ. Change, 44, 1-14, doi:10.1016/j.gloenvcha.2017.02.005.

Magnan, A.K. and T. Ribera, 2016: Global adaptation after Paris. Science, 352(6291), 1280-1282, doi:10.1126/science.aaf5002.

Maier, H.R. et al., 2016: An uncertain future, deep uncertainty, scenarios, robustness and adaptation: How do they fit together? Environ. Model. Soft., 81, 154-164, doi:10.1016/j.envsoft.2016.03.014.

Maldonado, J. et al., 2016: Engagement with indigenous peoples and honoring traditional knowledge systems. Clim. Change, 135(1), 111-126, doi:10.1007/s10584-015-1535-7.

Marchau, V.A.W.J., W.E. Walker, P.J.T.M. Bloemen and S.W. Popper, Eds., 2019a: Decision making under deep uncertainty: from theory to practice. Springer International Publishing, Cham, Switzerland, 400 pp. ISBN: 9783030052522.

Marchau, V.A.W.J., W.E. Walker, P.J.T.M. Bloemen and S.W. Popper, 2019b: Introduction. In: Decision making under deep uncertainty: From theory to practice [Marchau, V.A.W.J., W.E. Walker, P.J.T.M. Bloemen and S.W. Popper (eds.)]. Springer International Publishing, Cham, Switzerland, pp. 1-20. ISBN: 9783030052522.

Markham, A., E. Osipova, K. Lafrenz Samuels and A. Caldas, 2016: World heritage and tourism in a changing climate. United Nations Environment Programme, Nairobi, Kenya and United Nations Educational, Scientific and Cultural Organization, Paris, France, 104 pp. ISBN: 9789231001529.

Maru, Y.T. et al., 2014: A linked vulnerability and resilience framework for adaptation pathways in remote disadvantaged communities. Global Environ. Change, 28, 337-350, doi:10.1016/j.gloenvcha.2013.12.007.

Masson-Delmotte, V. et al., 2013: Information from paleoclimate archives. In: Climate Change 2013: The Physical Science Basis. Contribution of Working Group I to the Fifth Assessment Report of the Intergovernmental Panel on Climate Change [Stocker, T.F., D. Qin, G.-K. Plattner, M. Tignor, S.K. Allen, J. Boschung, A. Nauels, Y. Xia, V. Bex and P.M. Midgley (eds.)]. Cambridge University Press, Cambridge, United Kingdom and New York, NY, USA, 383-464.

Mastrandrea, M.C. et al., 2010: Guidance Note for Lead Authors of the IPCC Fifth Assessment Report on Consistent Treatment of Uncertainties. Intergovernmental Panel on Climate Change (IPCC) [Available at: www.ipcc.ch].

Matsumoto, K., 2007: Radiocarbon-based circulation age of the world oceans. J. Geophys. Res. Oceans, 112, C09004, doi:10.1029/2007JC004095.

Mayer, B. and F. Crépeau, 2017: Research Handbook on Climate Change, Migration and the Law. Edward Elgar Publishing, Cheltenham, UK, Northampton, MA, USA. ISBN: 9781785366581.

Mazloff, M.R., P. Heimbach and C. Wunsch, 2010: An eddy-permitting Southern Ocean state estimate. J. Phys. Oceanogr., 40(5), 880-899, doi:10.1175/2009JP04236.1.

McGuire, A.D. et al., 2018: Dependence of the evolution of carbon dynamics in the northern permafrost region on the trajectory of climate change. Proc. Natl. Acad. Sci. U.S.A.S, 115(15, 3882-3887, doi:10.1073/ pnas.1719903115.

McLeman, R.A. and L.M. Hunter, 2010: Migration in the context of vulnerability and adaptation to climate change: Insights from analogues. WIRes. Clim. Change, 1(3), 450-461, doi:10.1002/wcc.51.

McNamara, K.E. and R. Westoby, 2011: Solastalgia and the gendered nature of climate change: An example from Erub Island, Torres Strait. EcoHealth, 8(2), 233-236, doi:10.1007/s10393-011-0698-6. 
McQuatters-Gollop, A. et al., 2015: The Continuous Plankton Recorder survey: How can long-term phytoplankton datasets contribute to the assessment of Good Environmental Status? Estuarine, Coastal and Shelf Science, 162, 88-97, doi:10.1016/j.ecss.2015.05.010.

MEA, 2005: Ecosystems and human well-being: Synthesis. World Resources Institute, New Island Press, Washington, DC, 155 pp. ISBN: 9781597260404.

Mechler, R. et al., 2018: Loss and damage from climate change. Springer Berlin Heidelberg, New York NY. 557 pp. ISBN: 9783319720258.

Mekonnen, Z., H. Kassa, T. Woldeamanuel and Z. Asfaw, 2019: Analysis of observed and perceived climate change and variability in Arsi Negele District, Ethiopia. Environ. Dev. Sustain., 20(3), 1191-1212, doi:10.1007/ s10668-017-9934-8.

Merkens, J.L., L. Reimann, J. Hinkel and A.T. Vafeidis, 2016: Gridded population projections for the coastal zone under the Shared Socioeconomic Pathways. Glob. Planet. Change, 145, 57-66, doi:10.1016/j.gloplacha.2016.08.009.

Merrey, D.J. et al., 2018: Evolving high altitude livelihoods and climate change: A study from Rasuwa District, Nepal. Food Security, 10(4), 10551071, doi:10.1007/s12571-018-0827-y.

Mewhirter, J., M. Lubell and R. Berardo, 2018: Institutional externalities and actor performance in polycentric governance systems. Environmental Policy and Governance, 28(4), 295-307, doi:10.1002/eet.1816.

Milfont, T.L., M.S. Wilson and C.G. Sibley, 2017: The public's belief in climate change and its human cause are increasing over time. PloS ONE, 12 (3), e0174246, doi:10.1371/journal.pone.0174246.

Miller, D.D. et al., 2018: Adaptation strategies to climate change in marine systems. Glob. Chang. Biol., 24(1), e1-e14, doi:10.1111/gcb.13829.

Miloslavich, P. et al., 2018: Essential ocean variables for global sustained observations of biodiversity and ecosystem changes. Glob. Chang. Biol., 24(6), 2416-2433, doi:10.1111/gcb.14108.

Mistry, J. and A. Berardi, 2016: Bridging indigenous and scientific knowledge. Science, 352(6291), 1274-1275, doi:10.1126/science.aaf1160.

Molden, D.J., A.B. Shrestha, S. Nepal and W.W. Immerzeel, 2016: Downstream implications of climate change in the Himalayas. In: Water security, climate change and sustainable development. Springer, Singapore, pp. 65-82. ISBN: 9789812879769.

Moore, M.-L. et al., 2014: Studying the complexity of change: Toward an analytical framework for understanding deliberate social-ecological transformations. Ecol. Soc., 19(4), 54, doi:10.5751/ES-06966-190454.

Mora, C. et al., 2013: The projected timing of climate departure from recent variability. Nature, 502(7470), 183-187, doi:10.1038/nature12540.

Moser, S.C., 2016: Reflections on climate change communication research and practice in the second decade of the 21st century: What more is there to say? WIRes Clim. Change, 7(3), 345-369, doi:10.1002/wcc.403.

Moss, R. et al., 2008: Towards new scenarios for analysis of emissions, climate change, impacts and response strategies. IPCC Expert Meeting Report, 25 pp.

Moss, R.H. et al., 2010: The next generation of scenarios for climate change research and assessment. Nature, 463, 747-756, doi:10.1038/nature08823 www.nature.com/articles/nature08823\#supplementary-information.

Motta, M., 2018: The enduring effect of scientific interest on trust in climate scientists in the United States. Nat. Clim. Change, 8(6), 485-488, doi:10.1038/s41558-018-0126-9.

Mourey, J. and L. Ravanel, 2017: Evolution of Access Routes to High Mountain Refuges of the Mer de Glace Basin (Mont Blanc Massif, France): An Example of Adapting to Climate Change Effects in the Alpine High Mountains. Journal of Alpine Research | Revue de géographie alpine, 105(4), doi:10.4000/rga.3790.

Murakami, H. et al., 2018: Dominant effect of relative tropical Atlantic warming on major hurricane occurrence. Science, 362(6416), 794-799, doi:10.1126/science.aat6711.

Myers, T.A., M.C. Nisbet, E.W. Maibach and A.A. Leiserowitz, 2012: A public health frame arouses hopeful emotions about climate change. Clim. Change, 113(3-4), 1105-1112, doi:10.1007/s10584-012-0513-6.
Myhre, G. et al., 2013: Anthropogenic and natural radiative forcing. In: Climate Change 2013: The Physical Science Basis. Contribution of Working Group I to the Fifth Assessment Report of the Intergovernmental Panel on Climate Change [Stocker, T.F., D. Qin, G.-K. Plattner, M. Tignor, S.K. Allen, J. Boschung, A. Nauels, Y. Xia, V. Bex and P.M. Midgley (eds.)]. Cambridge University Press, Cambridge, United Kingdom and New York, NY, USA, 658-740.

Mysiak, J. et al., 2018: Brief communication: Strengthening coherence between climate change adaptation and disaster risk reduction. Nat. Hazar Earth Sys. Sci., 18(11), 3137-3143, doi:10.5194/nhess-18-3137-2018.

Nadeem, S., I. Elahi, A. Hadi and I. Uddin, 2012: Traditional knowledge and local institutions support adaptation to water-induced hazards in Chitral, Pakistan. ICIMOD, Kathmandu, 51 pp.

Nakashima, D. et al., 2012: Weathering uncertainty: Traditional knowledge for climate change assessment and adaptation. United Nations Educational, Scientific and Cultural Organization and United Nations University, Paris/ Darwin. 120 pp. ISBN: 9789230010683.

Nalau, J. et al., 2018: The role of indigenous and traditional knowledge in ecosystem-based adaptation: A review of the literature and case studies from the Pacific Islands. Weather Clim. Soc., 10(4), 851-865, doi:10.1175/ WCAS-D-18-0032.1.

National Academies of Sciences, Engineering, and Medicine, 2019: Negative Emissions Technologies and Reliable Sequestration: A Research Agenda. The National Academies Press, Washington, DC. ISBN: 9780309484527.

Neef, A. et al., 2018: Climate adaptation strategies in Fiji: The role of social norms and cultural values. World Dev., 107, 125-137, doi:10.1016/j. worlddev.2018.02.029.

Nickels, S., C. Furgal, M. Buell and H. Moquin, 2005: Putting the human face on climate change: Perspectives from Inuit in Canada. Joint publication of Inuit Tapiriit Kanatami, Nasivvik Centre for Inuit Health and Changing Environments at Université Laval and the Ajunnginiq Centre at the National Aboriginal Health Organization, Ottawa, 123 pp. ISBN: 9780969977414.

NOAA. 2018: National Centers for Environmental Information, 2018: State of the Climate: Hurricanes and Tropical Storms for Annual 2017. [Available at: www. ncdc.noaa.gov/sotc/tropical-cyclones/201713, accessed September 13].

North, D.C., 1990: A transaction cost theory of politics. J. Theor. Polit., 2(4), 355-367, doi:10.1177/0951692890002004001.

Nunn, P.D. and N.J. Reid, 2016: Aboriginal memories of inundation of the Australian coast dating from more than 7000 years ago. Aust. Geogr., 47(1), 11-47, doi:10.1080/00049182.2015.1077539.

Nüsser, M. and S. Schmidt, 2017: Nanga Parbat revisited: Evolution and dynamics of sociohydrological interactions in the Northwestern Himalaya. Ann. Am. Assoc. Geogr., 107(2), 403-415, doi:10.1080/24694452.2016.1 235495.

O'Neill, B.C. et al., 2017: IPCC reasons for concern regarding climate change risks. Nat. Clim. Change, 7(1), 28-37, doi:10.1038/nclimate3179.

O'Neill, B.C. et al., 2016: The scenario model intercomparison project (ScenarioMIP) for CMIP6. Geosci. Model Dev., 9(9), 3461-3482, doi:10.5194/gmd-9-3461-2016.

O'Neill, S.J. and S. Graham, 2016: (En)visioning place-based adaptation to sea-level rise. Geography and Environment, 3(2), 1-16, doi:10.1002/geo2.28.

O'Neill, B.C. et al., 2017: The roads ahead: Narratives for shared socioeconomic pathways describing world futures in the 21st century. Global Environ. Change, 42, 169-180, doi:10.1016/j.gloenvcha.2015.01.004.

Obermeister, N., 2017: From dichotomy to duality:Addressing interdisciplinary epistemological barriers to inclusive knowledge governance in global environmental assessments. Environmental Science \& Policy, 68, 80-86, doi:10.1016/j.envsci.2016.11.010.

Ogunbode, C.A. et al., 2018: The resilience paradox: Flooding experience, coping and climate change mitigation intentions. Clim. Policy, 19(6), 703715, doi:10.1080/14693062.2018.1560242.

Olsen, A. et al., 2016: The Global Ocean Data Analysis Project version 2 (GLODAPV2) - an internally consistent data product for the world ocean. Earth Syst. Sci. Data, 8(2), 297-323, doi:10.5194/essd-8-297-2016. 
Olsson, L. et al., 2017: Why resilience is unappealing to social science: Theoretical and empirical investigations of the scientific use of resilience. In: The Routledge Handbook of International Resilience [Chandler, D. and J. Coaffe (eds.)]. Routledge. 402 pp. ISBN: 9781138784321.

Olsson, L. et al., 2014: Livelihoods and poverty. In: Climate Change 2014: Impacts, Adaptation, and Vulnerability. Part A: Global and Sectoral Aspects. Contribution of Working Group II to the Fifth Assessment Report of the Intergovernmental Panel on Climate Change [Field, C.B., V.R. Barros, D.J. Dokken, K.J. Mach, M.D. Mastrandrea, T.E. Biller, M. Chatterjee, K.L. Ebi, Y.O. Estrada, R.C. Genova, B. Girma, E.S. Kissel, A.N. Levy, S. MacCracken, P.R. Mastrandrea and L.L. White (eds.)]. Cambridge University Press, Cambridge, United Kingdom and New York, NY, USA, 793-832.

Oppenheimer, M. et al., 2014: Emergent Risks and Key Vulnerabilities. In: Climate Change 2014: Impacts, Adaptation, and Vulnerability. Part A: Global and Sectoral Aspects. Contribution of Working Group II to the Fifth Assessment Report of the Intergovernmental Panel on Climate Change [Field, C.B., V.R. Barros, D.J. Dokken, K.J. Mach, M.D. Mastrandrea, T.E. Biller, M. Chatterjee, K.L. Ebi, Y.O. Estrada, R.C. Genova, B. Girma, E.S. Kissel, A.N. Levy, S. MacCracken, P.R. Mastrandrea and L.L. White (eds.)]. Cambridge University Press, Cambridge, United Kingdom and New York, NY, USA, 1039-1099.

Oschlies, A., P. Brandt, L. Stramma and S. Schmidtko, 2018: Drivers and mechanisms of ocean deoxygenation. Nat. Geosci., 11, 467-473, doi:10.1038/s41561-018-0152-2.

Ostrom, E., 2005: Understanding institutional diversity. Princeton University Press, Princeton, NJ, 355 pp. ISBN: 9780691122380.

Ostrom, E., 2010: Polycentric systems for coping with collective action and global environmental change. Global Environ. Change, 20(4), 550-557, doi:10.1016/j.gloenvcha.2010.07.004.

Otto, F.E., 2017:Attribution of weather and climate events. Annu. Rev. Environ. Resour., 42, 627-646, doi:10.1146/annurev-environ-102016-060847.

Paavola, J., 2007: Institutions and environmental governance: A reconceptualization. Ecol. Econ., 63(1), 93-103, doi:10.1016/j.ecolecon. 2006.09.026.

Pacific Community, 2016: Framework for resilient development in the Pacific: An integrated approach to address climate change and disaster risk management (FRDP). Pacific Community, Suva, Fiji, 32 pp. [Available at: http://gsd.spc.int/frdp/assets/FRDP_2016_Resilient_Dev_pacific.pdf].

Padmanabhan, M., 2017: Transdisciplinary Research and Sustainability: Collaboration, Innovation and Transformation. Routledge. 443 pp. ISBN: 9781138216402.

PAGES2K Consortium, 2017: A global multiproxy database for temperature reconstructions of the Common Era. Scientific Data, 4, 170088, doi:10.1038/sdata.2017.88.

Pahl-Wostl, C., 2019: The role of governance modes and meta-governance in the transformation towards sustainable water governance. Environmental Science \& Policy, 91, 6-16, doi:10.1016/j.envsci.2018.10.008.

Pahl-Wostl, C., A. Bhaduri and A. Bruns, 2018: Editorial special issue: The Nexus of water, energy and food - An environmental governance perspective. Environmental Science \& Policy, 90, 161-163, doi:10.1016/j. envsci.2018.06.021.

Palmer, M.D. and D.J. McNeall, 2014: Internal variability of Earth's energy budget simulated by CMIP5 climate models. Environ. Res. Letters, 9(3), 034016, doi:10.1088/1748-9326/9/3/034016.

Parmesan, C., T.L. Root and M.R. Willig, 2000: Impacts of extreme weather and climate on terrestrial biota. Bull. Am. Meteorol. Soc., 81(3), 443-450, doi:10.1175/1520-0477(2000)081<0443:IOEWAC>2.3.C0;2.

Parveen, S., M. Winiger, S. Schmidt and M. Nüsser, 2015: Irrigation in Upper Hunza: Evolution of socio-hydrological interactions in the Karakoram, northern Pakistan. Erdkunde, 69(1), 69-85, doi:10.3112/ erdkunde.2015.01.05.

Pelling, M., K. O'Brien and D. Matyas, 2015: Adaptation and transformation. Clim. Change, 133, 113-127, doi:10.1007/s10584-014-1303-0.
Pespeni, M.H. et al., 2013: Evolutionary change during experimental ocean acidification. Proc. Natl. Acad. Sci. U.S.A., 110(17), 6937-6942, doi:10.1073/pnas.1220673110.

Peters, G.P. et al., 2012: The challenge to keep global warming below $2^{\circ} \mathrm{C}$. Nat. Clim. Change, 3(1), 4-6, doi:10.1038/nclimate1783.

Petersen, A.C., 2011: Climate simulation, uncertainty, and policy advice-the case of the IPCC. In: Climate Change and Policy: The Calculability of Climate Change and the Challenge of Uncertainty [Gramelsberger, G. and J. Feichter (eds.)]. Springer, Berlin, Heidelberg, pp. 91-111. ISBN: 9783642177002.

Philip, T., 2018: Climate change displacement and migration: An analysis of the current international legal regime's deficiency, proposed solutions and a way forward for Australia. Melbourne Journal of International Law, 19, 27 pp.

Pierre, J. and G. Peters, 2000: Governance, Politics, and the State. St. Martin's Press. 240 pp. ISBN: 9780333718483.

Pincus, R. and S.H. Ali, 2015: Diplomacy on Ice: Energy and the Environment in the Arctic and Antarctic. Yale University Press, 304 pp. ISBN: 9780300205169.

Pistone, K., I. Eisenman and V. Ramanathan, 2014: Observational determination of albedo decrease caused by vanishing Arctic sea ice. Proc. Natl. Acad. Sci. U.S.A., 111(9), 3322-3326, doi:10.1073/pnas.1318201111.

Pörtner, H.-O. et al., 2014: Ocean Systems. In: Climate Change 2014: Impacts, Adaptation, and Vulnerability. Part A: Global and Sectoral Aspects. Contribution of Working Group II to the Fifth Assessment Report of the Intergovernmental Panel on Climate Change [Field, C.B., V.R. Barros, D.J. Dokken, K.J. Mach, M.D. Mastrandrea, T.E. Biller, M. Chatterjee, K.L. Ebi, Y.O. Estrada, R.C. Genova, B. Girma, E.S. Kissel, A.N. Levy, S. MacCracken, P.R. Mastrandrea and L.L. White (eds.)]. Cambridge University Press, Cambridge, United Kingdom and New York, NY, USA, 411-484.

Pratchett, M.S. et al., 2011: Vulnerability of coastal fisheries in the tropical Pacific to climate change. In: Vulnerability of Tropical Pacific Fisheries and Aquaculture to Climate Change [Bell, J.D., J.E. Johnson and A.J. Hobday (eds.)]. Secretariat of the Pacific Community, Noumea, pp. 493-576. ISBN: 9789820005082.

Prescott, S.L. and A.C. Logan, 2018: Larger than life: Injecting hope into the planetary health paradigm. Challenges, 9(13), 1-27, doi:10.3390/ challe9010013.

Przeslawski, R., M. Byrne and C. Mellin, 2015: A review and meta-analysis of the effects of multiple abiotic stressors on marine embryos and larvae. Glob. Chang. Biol., 21 (6), 2122-2140, doi:10.1111/gcb.12833.

PSMSL. Permanent Service for Mean Sea Level. [Available at: www.psmsl.org].

Purich, A. et al., 2018: Impacts of broad-scale surface freshening of the Southern Ocean in a coupled climate model. J. Clim., 31(7), 2613-2632, doi:10.1175/JCLI-D-17-0092.1.

Qian, H., R. Joseh and N. Zeng, 2010: Enhanced terrestrial carbon uptake in the Northern High Latitudes in the 21st century from the Coupled Carbon Cycle Climate Model Intercomparison Project model projections. Glob. Chang. Biol., 16(2), 641-656, doi:10.1111/j.1365-2486.2009.01989.x.

Quinlan, A.E., M. Berbés-Blázquez, L.J. Haider and G.D. Peterson, 2016: Measuring and assessing resilience: Broadening understanding through multiple disciplinary perspectives. J. Appl. Ecol., 53(3), 677-687, doi:10.1111/1365-2664.12550.

Ramm, T.D., C.J. White, A.H.C. Chan and C.S. Watson, 2017: A review of methodologies applied in Australian practice to evaulate long-term coastal adaptation options. Clim. Risk Manage., 17, 35-51, doi:10.1016/j. crm.2017.06.005.

Rasul, G., 2014: Food, water, and energy security in South Asia: A nexus perspective from the Hindu Kush Himalayan region. Environmental Science \& Policy, 39, 35-48, doi:10.1016/j.envsci.2014.01.010.

Raup, B.H., L.M. Andreassen, T. Bolch and S. Bevan, 2015: Remote Sensing of Glaciers. In: Remote Sensing of the Cryosphere [Tedesco, M. (ed.)]. Wiley Blackwell, pp. 123-156. ISBN: 9781118368855.

Redman, C.L., 1999: Human Impact on Ancient Environments. University of Arizona Press, Tuscon, 256 pp. ISBN: 9780816519637. 
Reid, J., 2013: Interrogating the neoliberal biopolitics of the sustainable development-resilience nexus. Int. Political Sociol., 7(4), 353-367, doi:10.1111/ips.12028.

Renaud, F.G. et al., 2015: Resilience and shifts in agro-ecosystems facing increasing sea-level rise and salinity intrusion in Ben Tre Province, Mekong Delta. Clim. Change, 133(1), 69-84, doi:10.1007/s10584-014-1113-4.

Renaud, F.G., U. Nehren, K. Sudmeier-Rieux and M. Estrella, 2016: Developments and Opportunities for Ecosystem-Based Disaster Risk Reduction and Climate Change Adaptation. In: Ecosystem-Based Disaster Risk Reduction and Adaptation in Practice [Renaud, F.G., K. Sudmeier-Rieux, M. Estrella and U. Nehren (eds.)]. Springer International Publishing, Switzerland, pp.1-20. ISBN: 9783319436319.

Rhein, M. et al., 2013: Observations: Ocean. In: Climate Change 2013: The Physical Science Basis. Contribution of Working Group I to the Fifth Assessment Report of the Intergovernmental Panel on Climate Change [Stocker, T.F., D. Qin, G.-K. Plattner, M. Tignor, S.K. Allen, J. Boschung, A. Nauels, Y. Xia, V.B. And and P.M. Midgley (eds.)]. Cambridge University Press, Cambridge, United Kingdom and New York, NY, USA, pp. 255-315.

Rhines, P., S. Häkkinen and S.A. Josey, 2008: Is oceanic heat transport significant in the climate system? In: Arctic-Subarctic Ocean Fluxes [Dickson, R.R., J. Meincke and P. Rhines (eds.)]. Springer, Dordrecht, pp. 87-109. ISBN: 9781402067730.

Riahi, K. et al., 2011: RCP 8.5 - a scenario of comparatively high greenhouse gas emissions. Clim. Change, 109, doi:10.1007/s10584-011-0149-y.

Riahi, K. et al., 2017:The Shared Socioeconomic Pathways and their energy, land use, and greenhouse gas emissions implications: An overview. Global Environ. Change, 42, 153-168, doi:10.1016/j.gloenvcha.2016.05.009.

Rice, W.R., 2002: Evolution of sex: Experimental tests of the adaptive significance of sexual recombination. Nat. Rev. Genet., 3(4), 241-251, doi:10.1038/nrg760.

Rigg, J. and K. Oven, 2015: Building liberal resilience? A critical review from developing rural Asia. Global Environ. Change, 32, 175-186, doi:10.1016/j. gloenvcha.2015.03.007.

Riser, S.C. et al., 2016: Fifteen years of ocean observations with the global Argo array. Nat. Clim. Change, 6(2), 145-153, doi:10.1038/nclimate2872.

Roberts, C.M. et al., 2017: Marine reserves can mitigate and promote adaptation to climate change. Proc. Natl. Acad. Sci. U.S.A., 114, 61676175, doi:10.1073/pnas.1701262114.

Rodgers, K., J. Lin and T. Frölicher, 2015: Emergence of multiple ocean ecosystem drivers in a large ensemble suite with an Earth system model. Biogeosciences, 12(11), 3301-3320, doi:10.5194/bg-12-3301-2015.

Roggero, M., A. Bisaro and S. Villamayor-Tomas, 2017: Institutions in the climate adaptation literature: A systematic literature review through the lens of the Institutional Analysis and Development framework. Journal of Institutional Economics, 14(3), 423-448, doi:10.1017/\$1744137417000376.

Roggero, M. et al., 2018: Introduction to the special issue on adapting institutions to climate change. Journal of Institutional Economics, 14(3), 409-422, doi:10.1017/S1744137417000649.

Rohe, J.R., S. Aswani, A. Schlüter and S.C. Ferse, 2017: Multiple drivers of local (non-) compliance in community-ased marine resource management: case studies from the South Pacific. Front. Mar. Sci., 4, 172, doi:10.3389/ fmars.2017.00172.

Romero-Lankao, P. et al., 2014: North America. In: Climate Change 2014: Impacts, Adaptation, and Vulnerability. Part B: Regional Aspects: Working Group II Contribution to the Fifth Assessment Report of the Intergovernmental Panel on Climate Change [Field, C.B., V.R. Barros, D.J. Dokken, K.J. Mach, M.D. Mastrandrea, T.E. Biller, M. Chatterjee, K.L. Ebi, Y.O. Estrada, R.C. Genova, B. Girma, E.S. Kissel, A.N. Levy, S. MacCracken, P.R. Mastrandrea and L.L. White (eds.)]. Cambridge University Press, Cambridge, United Kingdom and New York, NY, USA, 1439-1498.

Roncoli, C., T. Crane and B. Orlove, 2009: Fielding Climate Change in Cultural Anthropology. In: Anthropology and Climate Change: From Encounters to
Actions [Crate, S. and M. Nuttall (eds.)]. Left Coast Press, Walnut Creek, CA, pp. 87-115. ISBN: 9781598743340.

Roué, M., N. Césard, N.C. Adou Yao and A. Oteng-Yeboah, 2017: Knowing our lands and resources: Indigenous and local knowledge of biodiversity and ecosystem services in Africa. Knowledges of Nature 8, UNESCO, Paris. 156 pp. ISBN: 9789231002083.

Roué, M. and Z. Molnar, 2017: Knowing our lands and resources: indigenous and local knowledge of biodiversity and ecosystem services in Europe and Central Asia. Knowledges of Nature 9, UNESCO, Paris. 148 pp. ISBN: 9789231002106.

Roy, J., P. Tschakert, H. Waisman and S.A. Halim, 2018: Sustainable development, poverty eradication and reducing inequalities. In: Global Warming of $1.5^{\circ} \mathrm{C}$. An IPCC Special Report on the impacts of global warming of $1.5^{\circ} \mathrm{C}$ above pre-industrial levels and related global greenhouse gas emission pathways, in the context of strengthening the global response to the threat of climate change, sustainable development, and efforts to eradicate poverty [Masson-Delmotte, V., P. Zhai, H.O. Pörtner, D. Roberts, J. Skea, P.R. Shukla, A. Pirani, W. Moufouma-Okia, C. Péan, R. Pidcock, S. Connors, J.B.R. Matthews, Y. Chen, X. Zhou, M.I. Gomis, E. Lonnoy, T. Maycock, M. Tignor and T. Waterfield (eds.)]. World Meteorological Organization, Geneva, Switzerland.

Royal Society, 2018: Greenhouse Gas Removal. Royal Academy of Engineering, London, UK [Available at: https://royalsociety.org/ /media/ policy/projects/greenhouse-gas-removal/royal-society-greenhouse-ga s-removal-report-2018.pdf].

Ryan, P.A. and A. Münchow, 2017: Sea ice draft observations in Nares Strait from 2003 to 2012. J. Geophys. Res. Oceans, 122(4), 3057-3080, doi:10.1002/2016JC011966.

Sabel, C.F. and D.G. Victor, 2017: Governing global problems under uncertainty: Making bottom-up climate policy work. Clim. Change, 144(1), 15-27.

Sanderson, B.M., B.C. O'Neill and C. Tebaldi, 2016: What would it take to achieve the Paris temperature targets? Geophys. Res. Letters, 43(13), 7133-7142, doi:10.1002/2016GL069563.

Sarmiento, J.L. and N. Gruber, 2002: Sinks for anthropogenic carbon. Physics Today, 55(8), 30-36, doi:10.1063/1.1510279.

Sarmiento, J.L. and N. Gruber, 2006: Ocean Biogeochemical Dynamics. Princeton University Press, Princeton, New Jersey, 528 pp. ISBN: 9780691017075.

Savo, V. et al., 2016: Observations of climate change among subsistence-oriented communities around the world. Nat. Clim. Change, 6(5), 462-473, doi:10.1038/nclimate2958.

Schädel, C. et al., 2016: Potential carbon emissions dominated by carbon dioxide from thawed permafrost soils. Nat. Clim. Change, 6, 950-953, doi:10.1038/nclimate3054.

Schaum, C.E. and S. Collins, 2014: Plasticity predicts evolution in a marine alga. Proc. R. Soc. Lond. B., 281(1793), 20141486, doi:10.1098/rspb.2014.1486.

Schlosberg, D., L.B. Collins and S. Niemeyer, 2017: Adaptation policy and community discourse: Risk, vulnerability, and just transformation. Environ. Politics, 26(3), 413-437, doi:10.1080/09644016.2017.1287628.

Schurer, A.P. et al., 2017: Importance of the pre-industrial baseline for likelihood of exceeding Paris goals. Nat. Clim. Change, 7(8), 563-567, doi:10.1038/nclimate3345.

Seneviratne, S.I. et al., 2012: Summary for Policymakers. In: Managing the Risks of Extreme Events and Disasters to Advance Climate Change Adaptation. A Special Report of Working Groups I and II of the Intergovernmental Panel on Climate Change (IPCC). [Field, C.B., V. Barros, T.F. Stocker, D. Qin, D.J. Dokken, K.L. Ebi, M.D. Mastrandrea, K.J. Mach, G.-K. Plattner, S.K. Allen, M. Tignor and P.M. Midgley (eds.)]. Cambridge University Press, Cambridge, United Kingdom and New York, NY, USA, 109-230.

Serpetti, N. et al., 2017: Impact of ocean warming on sustainable fisheries management informs the ecosystem approach to fisheries. Sci. Rep., 7(13438), 15, doi:10.1038/s41598-017-13220-7. 
Shadian, J.M., 2014: The politics of Arctic sovereignty: oil, ice, and Inuit governance. Routledge.

Shaikh, F., Q. Ji and Y. Fan, 2015: The diagnosis of an electricity crisis and alternative energy development in Pakistan. Renew. Sust. Energ. Rev., 52, 1172-1185, doi:10.1016/j.rser.2015.08.009.

Shakhova, N. et al., 2013: Ebullition and storm-induced methane release from the East Siberian Arctic Shelf. Nat. Geosci., 7, 64-70, doi:10.1038/ ngeo2007.

Sharma, E. et al., 2019: Introduction. In: The Hindu Kush Himalaya Assessment - Mountains, Climate Change, Sustainability and People [Wester, P., A. Mishra, A. Mukherji and A.B. Shrestha (eds.)]. SpringerNature, Dordrecht. 627 pp. ISBN: 9783319950518.

Shepherd, J.G., P.G. Brewer, A. Oschlies and A.J.Watson, 2017: Ocean ventilation and deoxygenation in a warming world: Introduction and overview. Phil. Trans. Roy. Soc., 375, 20170240, doi:10.1098/rsta.2017.0240.

Sherpa, P., 2014: Climate change, perceptions, and social heterogeneity in Pharak, Mount Everest region of Nepal. Hum. Organ., 73(2), 153-161, doi:10.17730/humo.73.2.94q43152111733t6.

Shi, J., V.H.M. Visschers, M. Siegrist and J. Arvai, 2016: Knowledge as a driver of public perceptions about climate change reassessed. Nat. Clim. Change, 6(8), 759-762, doi:10.1038/nclimate2997.

Shi, J.-R., S.-P. Xie and L.D. Talley, 2018: Evolving relative importance of the southern ocean and North Atlantic in anthropogenic ocean heat uptake. J. Clim., 31(18), 7459-7479, doi:10.1175/JCLI-D-18-0170.1.

Shindell, D. et al., 2012: Simultaneously mitigating near-term climate change and improving human health and food security. Science, 335(6065), 183189, doi:10.1126/science.1210026.

Sigman, D. and E. Boyle, 2000: Glacial/interglacial variations in carbon dioxide. Nature, 407, 859-869, doi:10.1038/35038000.

Sillitoe, P., 2007: Local science vs. global science: Approaches to indigenous knowledge in international development. Studies in Environmental Anthropology and Ethnobiology, Berghahn Books, New York, Oxford. 300 pp. ISBN: 9781845456481.

Singh, G.G. et al., 2018: A rapid assessment of co-benefits and trade-offs among Sustainable Development Goals. Mar. Policy, 93, 223-231, doi:10.1016/j.marpol.2017.05.030.

Smith, E.K. and A. Mayer, 2019: Anomalous Anglophones? Contours of free market ideology, political polarization, and climate change attitudes in English-speaking countries, Western European and post-Communist states. Clim. Change, 152(1), 17-34, doi:10.1007/s10584-018-2332-x.

Smith, K.R. et al., 2014: Human health: impacts, adaptation, and co-benefits. In: Climate Change 2014: Impacts, Adaptation, and Vulnerability. Part A: Global and Sectoral Aspects. Contribution of Working Group II to the Fifth Assessment Report of the Intergovernmental Panel on Climate Change [Field, C.B., V.R. Barros, D.J. Dokken, K.J. Mach, M.D. Mastrandrea, T.E. Biller, M. Chatterjee, K.L. Ebi, Y.O. Estrada, R.C. Genova, B. Girma, E.S. Kissel, A.N. Levy, S. MacCracken, P.R. Mastrandrea and L.L. White (eds.)]. Cambridge University Press, Cambridge, United Kingdom and New York, NY, USA, 709-754.

Smith, N. and A. Leiserowitz, 2014: The role of emotion in global warming policy support and opposition. Risk Anal., 34(5), 937-948, doi:10.1111/ risa.12140.

Smith, N.D., S.I.F. Ndlovu and R.W. Summers, 2016: Milnerton Coast Legal Review: Legal Issues Relevant to the City of Cape Town's Coastal Erosion Management Strategy (for the portion of the Milnerton Coast that forms the study area), Prepared for ERMD, City of Cape Town. ERMD, Cape Town.

Solecki, W., M. Pelling and M. Garschagen, 2017: Transitions between risk management regimes in cities. Ecol. Soc., 22(2), 38, doi:10.5751/ES09102-220238.

Solomon, S., G.-K. Plattner, R. Knutti and P. Friedlingstein, 2009: Irreversible climate change due to carbon dioxide emissions. Proc. Natl. Acad. Sci. U.S.A., 106(6), 1704-1709, doi:10.1073/pnas.0812721106.
Sommerkorn, M. and A.E. Nilsson, 2015: Governance of Arctic ecosystem services. In: The Economics of Biodiversity and Ecosystem Services TEEB Scoping Study for the Arctic, ed. CAFF (Akureyri, Iceland: Conservation of Arctic Flora and Fauna, 2015), ISBN: 9789935431462.

Sowman, M., D. Scott and C. Sutherland, 2016: Governance and Social Justice Position Paper: Milnerton Beach, Prepared for ERMD, City of Cape Town. ERMD, Cape Town.

Spratt, D. and I. Dunlop, 2018: What lies beneath: The understatement of existential climate risk. Breakthrough-National Centre for Climate Restoration, Melbourne, Australia, $40 \mathrm{pp}$.

Steffen, W. et al., 2018: Trajectories of the Earth System in the Anthropocene. Proc. Natl. Acad. Sci. U.S.A., 115(33), 8252-8259, doi:10.1073/ pnas. 1810141115.

Steiner, C.E., 2015: A sea of warriors: Performing an identity of resilience and empowerment in the face of climate change in the Pacific. The Contemporary Pacific, 27(1), 147-180, doi:10.1353/cp.2015.0002.

Storto, A. et al., 2018: Extending an oceanographic variational scheme to allow for affordable hybrid and four-dimensional data assimilation. Ocean Model., 128, 67-86, doi:10.1016/j.ocemod.2018.06.005.

Stott, P.A. et al., 2016: Attribution of extreme weather and climate-related events. WIRes Clim. Change, 7(1), 23-41, doi:10.1002/wcc.380.

Strang, V., 2009: Integrating the social and natural sciences in environmental research: A discussion paper. Environ. Dev. Sustain., 11(1), 1-18, doi:10.1007/s10668-007-9095-2.

Stroeve, J.C. et al., 2017: Investigating the local-scale influence of sea ice on Greenland surface melt. The Cryosphere, 11(5), 2363-2381, doi:10.5194/ tc-2017-65.

Sud, R., A. Mishra, N. Varma and S. Bhadwal, 2015: Adaptation policy and practice in densely populated glacier-fed river basins of South Asia: A systematic review. Reg. Environ. Change, 15 (5), 825-836, doi:10.1007/ s10113-014-0711-z.

Sunday, J.M. et al., 2014: Evolution in an acidifying ocean. Trends Ecol. Evol., 29(2), 117-125, doi:10.1016/j.tree.2013.11.001.

Sutton, R.T., 2018: Ideas: A simple proposal to improve the contribution of IPCC WG1 to the assessment and communication of climate change risks. Earth Syst. Dynam., 9, 1155-1158, doi:10.5194/esd-2018-36.

Szabo, S. et al., 2016: Making SDGs work for climate change hotspots. Environment: Science Policy for Sustainable Development, 58 (6), 24-33, doi:10.1080/00139157.2016.1209016.

Tagliabue, A. et al., 2017: The integral role of iron in ocean biogeochemistry. Nature, 543(7643), 51-59, doi:10.1038/nature21058.

Talley, L.D., 2013: Closure of the global overturning circulation through the Indian, Pacific, and Southern Oceans: Schematics and transports. Oceanography, 26(1), 80-97, doi:10.5670/oceanog.2013.07.

Talley, L.D. et al., 2016: Changes in ocean heat, carbon content and ventilation: A review of the first decade of GO-SHIP global repeat hydrography. Annu. Rev.Mar.Sci. 8(1), 185-215, doi:10.1146/annurev-marine-052915-100829.

Tarnocai, C. et al., 2009: Soil organic carbon pools in the northern circumpolar permafrost region. Global Biogeochem. Cycles, 23(2), 1-11, doi:10.1029/2008GB003327.

Tasmanian Climate Change Office, 2017: Tasmanian Wilderness and World Heritage Area Bushfire and Climate Change Research Project: Tasmanian Government's Response. Tasmanian Climate Change Office Department of Premier and Cabinet, 49 pp. ISBN: 9780724656456.

Taylor, K.E., R.J. Stouffer and G.A. Meehl, 2012: An overview of CMIP5 and the experiment design. Bull. Am. Meteorol. Soc., 93(4), 485-498, doi:10.1175/ BAMS-D-11-00094.1.

Thaman, R. et al., 2013: The contribution of indigenous and local knowledge systems to IPBES: Building synergies with science. IPBES Expert Meeting Report, UNESCO/UNU, Paris, UNESCO, 49 pp.

Thomas, K. et al., 2019: Explaining differential vulnerability to climate change: A social science review. WIRes Clim. Change, 10(2), 1-18, doi:10.1002/ wcc.565. 
Thomson, J. andW.E. Rogers, 2014: Swell and sea in the emerging Arctic Ocean. Geophys. Res. Letters, 41(9), 3136-3140, doi:10.1002/2014GL059983.

Thornton, B.F. et al., 2016: Methane fluxes from the sea to the atmosphere across the Siberian shelf seas. Geophys. Res. Letters, 43(11), 5869-5877, doi:10.1002/2016GL068977.

Tierney, K., 2015: Resilience and the neoliberal project: Discourses, critiques, practices - and Katrina. Am. Behav. Sci, 59(10), 1327-1342, doi:10.1177/0002764215591187.

Townhill, B. et al., 2017: Non-native marine species in north-west Europe: Developing an approach to assess future spread using regional downscaled climate projections. Aquat. Conserv., 27(5), 1035-1050, doi:10.1002/ aqc. 2764.

Trenberth, K.E., J.T. Fasullo and M.A. Balmaseda, 2014: Earth's Energy Imbalance. J. Clim., 27 (9), 3129-3144, doi:10.1175/JCLI-D-13-00294.1.

Trenberth, K.E., J.T. Fasullo and T.G. Shepherd, 2015: Attribution of climate extreme events. Nat. Clim. Change, 5, 725-730, doi:10.1038/nclimate2657.

Trenberth, K.E. et al., 2007: Estimates of the global water budget and its annual cycle using observational and model data. J. Hydrometeorol., 8, 758-769, doi:10.1175/JHM600.1.

Trusel, L.D. et al., 2018: Nonlinear rise in Greenland runoff in response to post-industrial Arctic warming. Nature, 564 (7734), 104-108, doi:10.1038/ s41586-018-0752-4.

Tschakert, P. et al., 2017: Climate change and loss, as if people mattered: Values, places, and experiences. WIRes Clim. Change, 8(5), e476, doi:10.1002/wcc. 476 .

UN, 1992: United Nations Framework Convention on Climate Change. FCCCI INFORMAL/84, United Nations, New York, 24.

UN, 2015a: Small Island Developing States in Numbers, Climate Change. Edition 2015. United Nations, New York, [Available at: http://unohrlls.org/ small-island-developing-states-in-numbers-2015/].

UN, 2015b: United Nations General Assembly. Transforming our world: The 2030 Agenda for Sustainable Development. A/RES/70/1 [Available at: www.un.org/sustainabledevelopment/development-agenda/].

UNFCCC, 2015: The Paris Agreement. (FCCC/CP/2015/10/Add.1).

Unsworth, K.L. and K.S. Fielding, 2014: It's political: How the salience of one's political identity changes climate change beliefs and policy support. Global Environ. Change, 27, 131-137, doi:10.1016/j.gloenvcha.2014.05.002.

Uprety, K. and S.M. Salman, 2011: Legal aspects of sharing and management of transboundary waters in South Asia: Preventing conflicts and promoting cooperation. Hydrolog. Sci. J., 56(4), 641-661, doi:10.1080/02626667.20 11.576252

van Oppen, M.J.H., J.K. Oliver, H.M. Putnam and R.D. Gates, 2015: Building coral reef resilience through assisted evolution. Proc. Natl. Acad. Sci. U.S.A., 112, 2307-2313, doi:10.1073/pnas.1422301112.

van Ruijven, B.J. et al., 2014: Enhancing the relevance of Shared Socioeconomic Pathways for climate change impacts, adaptation and vulnerability research. Clim. Change, 122(3), 481-494, doi:10.1007/s10584-013-0931-0.

van Valkengoed, A.M. and L. Steg, 2019: Meta-analyses of factors motivating climate change adaptation behaviour. Nat. Clim. Change, 9 (2), 158-163, doi:10.1038/s41558-018-0371-y.

van Vuuren, D.P. et al., 2011a: The representative concentration pathways: An overview. Clim. Change, 109 (1-2), 5-31, doi:10.1007/s10584-011-0148-z.

van Vuuren, D.P. et al., 2011b: RCP2.6: exploring the possibility to keep global mean temperature increase below $2^{\circ} \mathrm{C}$. Clim. Change, 109, 95-116, doi:10.1007/s10584-011-0152-3.

Varma, N. et al., 2014: Climate change, disasters and development: Testing the waters for adaptive governance in India. Vision: The Journal of Business Perspective, 18(4), 327-338, doi:10.1177/0972262914551664.

Vaughan, D.G. et al., 2013: Observations: Cryosphere. In: Climate Change 2013: The Physical Science Basis. Contribution of Working Group I to the Fifth Assessment Report of the Intergovernmental Panel on Climate Change [Stocker, T.F., D. Qin, G.-K. Plattner, M. Tignor, S.K. Allen, J. Boschung,
A. Nauels, Y. Xia, V. Bex and P.M. Midgley (eds.)]. Cambridge University Press, Cambridge, United Kingdom and New York, NY, USA, 317-382.

Vidas, D., 2000: Protecting the Polar marine environment: Law and policy for pollution prevention. Cambridge University Press, Cambridge, United Kingdom, 276 pp. ISBN: 0521663113.

Vidas, D., D. Freestone and J. McAdam, 2015: International law and sea level rise: The new ILA committee. ILSA Journal of International \& Comparative Law, 21(2), 397-408.

Vierros, M., 2017: Communities and blue carbon: The role of traditional management systems in providing benefits for carbon storage, biodiversity conservation and livelihoods. Clim. Change, 140(1), 89-100, doi:10.1007/ s10584-013-0920-3.

Visbeck, M., 2018: Ocean science research is key for a sustainable future. Nature Commun., 9(1), 690, doi:10.1038/s41467-018-03158-3.

von Schuckmann, K. et al., 2016: An imperative to monitor Earth's energy imbalance. Nat. Clim. Change, 6, 138-144, doi:10.1038/nclimate2876.

Walker, B., C.S. Holling, S. Carpenter and A. Kinzig, 2004: Resilience, adaptability and transformability in social-ecological systems. Ecol. Soc., 9(2), 5.

Walter, A.M., 2014: Changing Gilgit-Baltistan: Perceptions of the recent history and the role of community activism. Ethnoscripts, 16(1), 31-49.

Warner, J.F., 2016: Of river linkage and issue linkage: Transboundary conflict and cooperation on the River Meuse. Globalizations, 13(6), 741-766.

Warner, K. and K. van der Geest, 2013: Loss and damage from climate change: Local-level evidence from nine vulnerable countries. Int. J. Global Warm., 5(4), 367-386, doi:10.1504/JJGW.2013.057289.

Watt-Cloutier, S., 2018: The right to be cold: One woman's fight to protect the Arctic and save the planet from climate change. University of Minnesota Press, Minneapolis; London, 368 pp. ISBN: 9780670067107.

Weaver, C. et al., 2017: Reframing climate change assessments around risk: Recommendations for the US National Climate Assessment. Environ. Res. Letters, 12 (8), 080201, doi:10.1088/1748-9326/aa7494.

Weichselgartner, J. and I. Kelman, 2014: Geographies of resilience: Challenges and opportunities of a descriptive concept. Prog. Hum. Geogr., 39(3), 249267, doi:10.1177/0309132513518834.

West, J.J. and G.K. Hovelsrud, 2010: Cross-scale adaptation challenges in the coastal fisheries: Findings from Lebesby, Northern Norway. Arctic, 63(3), 338-354, doi:10.2307/20799601.

WGMS, 2017: Fluctuations of Glaciers Database. World Glacier Monitoring Service, Zurich, Switzerland [Available at: http://dx.doi.org/10.5904/ wgms-fog-2017-10].

Wise, R.M. et al., 2014: Reconceptualising adaptation to climate change as part of pathways of change and response. Global Environ. Change, 28, 325-336, doi:10.1016/j.gloenvcha.2013.12.002.

WOA, 2016: The First Global Integrated Marine Assessment: World Ocean Assessment I. Cambridge University Press, Cambridge, United Kingdom, 973 pp. ISBN: 9781108186148.

Wohling, M., 2009: The problem of scale in indigenous knowledge: A perspective from northern Australia. Ecol. Soc., 14(1), 1.

Wong, P.P. et al., 2014: Coastal systems and low-lying areas. In: Climate Change 2014: Impacts, Adaptation, and Vulnerability. Part A: Global and Sectoral Aspects. Contribution of Working Group II to the Fifth Assessment Report of the Intergovernmental Panel on Climate Change [Field, C.B., V.R. Barros, D.J. Dokken, K.J. Mach, M.D. Mastrandrea, T.E. Bilir, M. Chatterji, K.L. Ebi, Y.O. Estrada, R.C. Genova, B. Girma, E.S. Kissel, A.N. Levy, S. MacCracken, P.R. Mastrandrea and L.L. White (eds.)]. Cambridge University Press, Cambridge, United Kingdom and New York, NY, USA, 361-409.

Wunsch, C., P. Heimbach, R. Ponte and I. Fukumori, 2009: The global general circulation of the ocean estimated by the ECCO-Consortium. Oceanography, 22(2), 88-103, doi:10.5670/oceanog.2009.41.

Wymann von Dach, S. et al., 2018: Leaving no one in mountains behind: localizing the SDGs for resilience of mountain people and ecosystems. Issue Brief 2018: Sustainable Mountain Development Centre for Development 
and Environment and Mountain Research Initiative, with Bern Open Publishing (BOP), Bern, Switzerland. $12 \mathrm{pp}$.

$\mathrm{Xu}, \mathrm{Y}$. and V. Ramanathan, 2017: Well below $2^{\circ} \mathrm{C}$ : Mitigation strategies for avoiding dangerous to catastrophic climate changes. PNAS, 114(39), 10315-10323, doi:10.1073/pnas.1618481114.

Yager, K., 2015: Satellite imagery and community perceptions of climate change impacts and landscape change. In: Climate Cultures: Anthropological Perspectives on Climate Change [Jessica, B. and M.R. Dove (eds.)]. Yale University Press, New Haven, pp. 146-168. ISBN: 9780300198812.

Yeh, E.T., 2016: How can experience of local residents be "knowledge"? Challenges in interdisciplinary climate change research. Area, 48(1), 34-40, doi:10.1111/area.12189.

Young, O.R., 2016: The shifting landscape of Arctic politics: Implications for international cooperation. The Polar Journal, 6(2), 209-223, doi:10.1080/ 2154896X.2016.1253823.

Yu, L., 2018: Global air-sea fluxes of heat, fresh water, and momentum: Energy budget closure and unanswered questions. Annu. Rev. Mar. Sci., 11, 227-248, doi:10.1146/annurev-marine-010816-060704.

Zheng, J. and S. Suh, 2019: Strategies to reduce the global carbon footprint of plastics. Nat. Clim. Change, 9, 374-378, doi:10.1038/s41558-019-0459-z.

Zscheischler, J. et al., 2018: Future climate risk from compound events. Nat. Clim. Change, 8(6), 469-477, doi:10.1038/s41558-018-0156-3.

Zuo, H., M.A. Balmaseda, K. Mogensen and S. Tietsche, 2018: OCEAN5: The ECMWF Ocean Reanalysis System and its Real-Time analysis component. ECMWF Tech Memo 823. 
https://doi.org/10.1017/9781009157964.003 Published online by Cambridge University Press 